\title{
GERBAL REPRESENTATIONS OF DOUBLE LOOP GROUPS
}

\author{
EDWARD FRENKEL AND XINWEN ZHU
}

\begin{abstract}
A crucial role in representation theory of loop groups of reductive Lie groups and their Lie algebras is played by their non-trivial second cohomology classes which give rise to their central extensions (the affine Kac-Moody groups and Lie algebras). Loop groups embed into the group $G L_{\infty}$ of continuous automorphisms of $\mathbb{C}((t))$, and these classes come from a second cohomology class of $G L_{\infty}$. In a similar way, double loop groups embed into a group of automorphisms of $\mathbb{C}((t))((s))$, denoted by $G L_{\infty, \infty}$, which has a non-trivial third cohomology. In this paper we explain how to realize a third cohomology class in representation theory of a group: it naturally arises when we consider representations on categories rather than vector spaces. We call them "gerbal representations." We then construct a gerbal representation of $G L_{\infty, \infty}$ (and hence of double loop groups), realizing its non-trivial third cohomology class, on a category of modules over an infinite-dimensional Clifford algebra. This is a two-dimensional analogue of the fermionic Fock representations of the ordinary loop groups.
\end{abstract}

\section{Contents}

Introduction

1. 1-dimensional story: Projective representations 6

1.1. Homological constructions

1.2. Geometric constructions: non-linear version 9

1.3. Algebraic constructions: linear version 13

2. Generalities on the actions of groups on categories 15

2.1. 2-groups 16

2.2. Central extensions 17

2.3. Actions of groups on categories $\quad 17$

3. 2-dimensional story: Gerbal representations 22

3.1. The Lie algebra $\mathfrak{g l}_{\infty, \infty}$ and the group $G L_{\infty, \infty} \quad 22$

3.2. A gerbal representation of $G L_{\infty, \infty}$ on a category of Clifford modules 25

3.3. The 2-group determinantal extension of $G L_{\infty, \infty} \quad 30$

3.4. 2-infinite Grassmannian 33

4. Cohomology of $G L_{\infty, \infty}$ and related groups 35

4.1. Lie algebras and Lie groups of matrices

4.2. Computation of group cohomology 37

5. Cohomology classes of gerbal representations of $G L_{\infty, \infty} \quad 44$

5.1. Gerbal pairs of groups 44

5.2. Gerbal pairs associated with $G L_{\infty, \infty} \quad 51$

5.3. Computation of the cohomology classes of gerbal representations

References $\quad 59$

Date: October 2008.

Supported by DARPA and AFOSR through the grant FA9550-07-1-0543. 


\section{INTRODUCTION}

The main motivation for this paper and its sequel [FZ] is to understand representation theory of double loop groups and the corresponding Lie algebras and the role played in it by their third cohomology.

To explain this, we revisit representation theory of the ordinary loop groups. Its algebraic version is the formal loop group $G((t))$ of a reductive complex algebraic group $G$. The corresponding Lie algebra is the formal loop algebra $\mathfrak{g}((t))$, where $\mathfrak{g}=\operatorname{Lie} G$. A great discovery made over thirty years ago was the realization that in order to treat its representation theory "in the right way", we need to pass from $\mathfrak{g}((t))$ to its universal central extension, the affine Kac-Moody algebra $\widehat{\mathfrak{g}}$ (and similarly for the loop group). These central extensions (for different $\mathfrak{g}$ ) may be obtained in a unified way from the central extension of the "master" Lie algebra $\mathfrak{g l}_{\infty}$ of continuous endomorphisms of $\mathbb{C}((t))$. It is known [FT1 that $H^{\bullet}\left(\mathfrak{g l}_{\infty}, \mathbb{C}\right)=\mathbb{C}\left[c_{2}, c_{4}, \ldots\right]$ with $\operatorname{deg} c_{2 n}=2 n$, and so $H^{2}\left(\mathfrak{g l}_{\infty}, \mathbb{C}\right)=\mathbb{C} c_{2}$. The corresponding two-cocycle defines the universal central extension $\mathfrak{g l}_{\infty}$ of $\mathfrak{g l}_{\infty}$. For each finite-dimensional representation $V$ of $\mathfrak{g}$ we have a natural embedding of $\mathfrak{g}((t))$ into $\mathfrak{g l}_{\infty}$ (as endomorphisms of $V((t)) \simeq \mathbb{C}((t))$ ), and the pull-back of the universal central extension of $\mathfrak{g l}_{\infty}$ gives rise to $\widehat{\mathfrak{g}}$. In the same way one obtains the Virasoro algebra and its semi-direct products with $\widehat{\mathfrak{g}}$.

Representation theory of these groups and Lie algebras begins in earnest when we give an example of a natural representation with a non-zero action of the central element. The representation that is the easiest to construct is the fermionic Fock representation of $\widehat{\mathfrak{g l}}_{\infty}[\mathrm{KP}$. Its restriction to $\widehat{\mathfrak{g}}$ gives rise to important integrable representations Fre, $\mathrm{KP}$, and we have the beginnings of the general theory $[\mathrm{K}]$.

The fermionic Fock representation may also be constructed geometrically, as the space of global sections of the determinant line bundle on the infinite Grassmannian Gr which parametrizes "lattices" in the infinite-dimensional topological vector space $\mathbb{C}((t))$. The Lie algebra $\mathfrak{g l}_{\infty}$ and the corresponding group $G L_{\infty}$ naturally act on Gr. The important point is that this action does not lift to the determinant line bundle; it is only the central extension that acts on this line bundle.

Now let us consider the formal double loop group $G((t))((s))$ and its Lie algebra $\mathfrak{g}((t))((s))$ 1] Just like ordinary loop algebras, these Lie algebras naturally embed into another "master" Lie algebra, $\mathfrak{g l}_{\infty, \infty}$, which consists of continuous endomorphisms of $\mathbb{C}((t))((s))$. Hence in order to develop representation theory of double loop algebras it is natural to start with $\mathfrak{g l}_{\infty, \infty}$ and see what kinds of representations we can construct. By analogy with loop algebras, we look at the cohomology of $\mathfrak{g l}_{\infty, \infty}$. It is now the exterior algebra $\bigwedge\left(e_{3}, e_{5}, \ldots\right)$ with $\operatorname{deg} e_{2 n+1}=2 n+1$ [FT1]. In particular, the second cohomology that is responsible for central extensions vanishes, and the first non-trivial cohomology class $e_{3}$ occurs in degree three.

This cohomology class, in turn, gives rise to third cohomology classes of all double loop algebras. One can show that this cohomology class is non-zero for $\mathfrak{g}=\mathfrak{s l}_{n}, n>2$, as well as for $\mathfrak{g}=\mathfrak{g l}_{1}$ (see [Fe]). We also obtain third cohomology classes of the Lie algebra of derivations of $\mathbb{C}((t))((s))$ and its semi-direct products with $\mathfrak{g}((t))((s))$, which

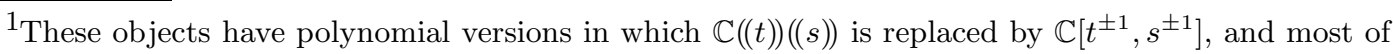
our results have analogues for these polynomial versions. However, we focus in this paper on the case of the formal power series, because the corresponding theory seems more natural.
} 
also embed naturally into $\mathfrak{g l}_{\infty, \infty}$. We wish to interpret these cohomology classes from the representation theoretic point of view ${ }^{2}$

To contrast representations of $\mathfrak{g l}_{\infty}$ and $\mathfrak{g l}_{\infty, \infty}$ in more concrete terms, let us recall that the naive action of $\mathfrak{g l}_{\infty}$ on the fermionic Fock representation creates infinite expressions, which need to be regularized by imposing "normal ordering" (see, e.g., [FB]). This normal ordering creates an "anomaly": commutation relations get distorted by additional terms which are interpreted as a central extension of $\mathfrak{g l}_{\infty}$. If we try to imitate this procedure in the case of $\mathfrak{g l}_{\infty, \infty}$, the result is much worse: not only the commutation relations get distorted, but the additional terms themselves turn out to be infinite. The anomaly is thus much more severe in the case of $\mathfrak{g l}_{\infty, \infty}$ than in the case of $\mathfrak{g l}_{\infty}$. It cannot possibly be cured by normal ordering alone. In fact, the cohomology class related to this anomaly has now migrated from $H^{2}$ to $H^{3}$.

How can we possibly interpret this third cohomology class in representation theory? The idea is that these classes are naturally realized when groups (or Lie algebras) act on categories rather than vector spaces. (Informally, one can say that the structure of a category is needed to absorb the $H^{3}$-anomaly discussed above.) To see how this works, we first recall how the second cohomology class is realized.

Suppose that $G$ is a group and $V$ is a complex vector space. To define a representation of $G$ on $V$ (here, for simplicity, we consider $G$ as an abstract group; but we extend this to algebraic groups and Lie algebras below and in [FZ]), we need to assign to each $g \in G$ a linear operator $T_{g}$ on $V$, so that $1 \in G$ goes to the identity, and for each pair $g, h \in G$ we have the equality $T_{g h}=T_{g} T_{h}$. We generalize this by relaxing the last condition and demanding only that

$$
T_{g h}=\alpha_{g, h} T_{g} T_{h},
$$

where $\alpha_{g, h} \in \mathbb{C}^{\times}$. Thus, we arrive at the notion of projective representation of $G$, or equivalently, a representation of the central extension of $G$ corresponding to $\alpha_{g, h}$ (one checks easily that it defines a two-cocycle of $G$ with coefficients in $\mathbb{C}^{\times}$).

Now we generalize this as follows. We replace a complex vector space $V$ by an abelian category $\mathcal{C}$ over $\mathbb{C}$. A representation of $G$ on $\mathcal{C}$ is a rule that assigns to each $g \in G$ a functor $F_{g}$ so that $1 \in G$ goes to the identity functor. For each pair $g, h \in G$ we then have two functors, $F_{g h}$ and $F_{g} \circ F_{h}$. Functors are objects of a category (rather than a set), and therefore it is not a good idea to demand that they be equal. Rather, we should demand that they are isomorphic. So we include the data of isomorphisms $i_{g, h}: F_{g} \circ F_{h} \stackrel{\sim}{\longrightarrow} F_{g h}$ in our definition. Suppose now that we have three elements $g, h, k \in G$. Then we have two different isomorphisms between $F_{g} \circ F_{h} \circ F_{k}$ and $F_{g h k}$; namely, $i_{g, h k} \circ i_{h, k}$ and $i_{g h, k} \circ i_{g, h}$. These are already elements of a set (that of morphisms from $F_{g} \circ F_{h} \circ F_{k}$ to $\left.F_{g h k}\right)$. Demanding that

$$
i_{g, h k} \circ i_{h, k}=i_{g h, k} \circ i_{g, h},
$$

we obtain an analogue of an ordinary representation of $G$ on a vector space. Alternatively, we may demand that this equality is only satisfied up to a non-zero scalar:

$$
i_{g, h k} \circ i_{h, k}=\alpha_{g, h, k} i_{g h, k} \circ i_{g, h}, \quad \alpha_{g, h k} \in \mathbb{C}^{\times} .
$$

\footnotetext{
${ }^{2}$ Note that double loop algebras also have non-trivial (actually, infinite-dimensional) second cohomology. Representations of the corresponding universal central extension have been studied in the literature; see, e.g., MRY, La, BBS, Bi].
} 
It is easy to check that $\alpha_{g, h, k}$ defines a three-cocycle of $G$ with coefficients in $\mathbb{C}^{\times}$. It is non-trivial if and only if our representation of $G$ on $\mathcal{C}$ is not equivalent to an ordinary representation (that is, one with $\alpha_{g, h, k} \equiv 1$ ), in the obvious sense. Moreover, one can check that if two representations of $G$ on $\mathcal{C}$ are isomorphic, in a natural sense, then the corresponding two cocycles differ by a coboundary.

Thus, we obtain a natural realization of the third cohomology when we consider representations of groups on categories. This notion may also be generalized to Lie algebras, as we explain in [FZ. We call representations of this type gerbal representations.

The idea that a group or a Lie algebra should act on a category rather than a vector space is not new. In recent years representations of groups on categories have naturally arisen in different contexts. For instance, in the work of D. Gaitsgory and one of the authors [FG] (see $[\mathrm{Fr}$. for an exposition) categories with an action of the loop group of a reductive algebraic group $G$ naturally arise in the framework of local geometric Langlands correspondence (in this case the third cohomology class is trivial). Another class of examples is presumably provided by various categories of branes arising in topological field theory. The group of symmetries of the theory should act on such a category. Moreover, the data of non-trivial third cohomology classes may be naturally included in this context (see, e.g., $[\mathrm{F}$ and references therein). One can probably use these data to construct gerbal representations of groups on categories of branes realizing these cohomology classes, though so far we have not seen examples of such representations discussed in the literature.

The goal of this paper is to construct explicitly non-trivial examples of gerbal representations of the group $G L_{\infty, \infty}$. In [FZ] we will construct gerbal representations of the Lie algebra $\mathfrak{g l}_{\infty, \infty}$. One of the gerbal representations of $G L_{\infty, \infty}$ that we construct may be thought of as an analogue of the fermionic Fock representation of $\mathfrak{g l}_{\infty}$. The corresponding category may be realized as the category of Fock representations of a Clifford algebra built from the vector space $\mathbb{C}((t))[[s]]$ plus its dual. We compute explicitly the third cohomology class of $G L_{\infty, \infty}$ corresponding to this representation and show that it is non-zero. It gives rise to a non-trivial central extension of $G L_{\infty, \infty}$ by $B \mathbb{C}^{\times}$(see Definition 2.5) considered previously by S. Arkhipov and K. Kremnizer in [AK, following ideas of M. Kapranov. Thus, we obtain a genuine representation of this extension on a category of representations of this Clifford algebra.

For any reductive group $G$ and a finite-dimensional representation $V$ of $G$ we have a natural embedding of the double loop group $G((t))((s))$ into $G L_{\infty, \infty}$. Hence our gerbal representation of $G L_{\infty, \infty}$ gives rise to gerbal representations of $G((t))((s))$. The corresponding cohomology class is the restriction of the third cohomology class of $G L_{\infty, \infty}$ to $G((t))((s))$. We expect that this restriction is non-zero if and only if $H^{6}(B G, \mathbb{Z})$ is nonzero. This is the case, for example, for the groups $G L_{1}, S L_{n}, n>2$, and $G S p_{2 n}, n>1$. (This is analogous to the fact that the central extensions of $G((t))$ are classified by $H^{4}(B G, \mathbb{Z})$.)

We expect that gerbal representations of $G L_{\infty, \infty}$ may also be constructed geometrically, using a "2-infinite Grassmannian". Set-theoretically, this is just the set of lattices in $\mathbb{C}((t))((s))$. The natural transitive action of $G L_{\infty, \infty}$ on this set lifts to the action of the above $B \mathbb{C}^{\times}$-extension of $G L_{\infty, \infty}$ on a $\mathbb{C}^{\times}$-gerbe over it (see also [AK]). This is analogous to the action of $\widehat{G L}_{\infty}$ on the $\mathbb{C}^{\times}$-bundle over the infinite Grassmannian. Recall that we obtain a representation of $\widehat{G L}_{\infty}$ by taking the vector space of global 
sections of the corresponding determinant line bundle. If one could define the 2-infinite Grassmannian and the $\mathbb{C}^{\times}$-gerbe over it algebro-geometrically, then the category of "global sections" of the sheaf of abelian categories corresponding to the $\mathbb{C}^{\times}$-gerbe (the way a line bundle corresponds to a $\mathbb{C}^{\times}$-bundle) would give us a gerbal representation of $G L_{\infty, \infty}$. This is still an open question, but we make some comments on how to answer it 93.4 .

We hope that the gerbal representations of $G L_{\infty, \infty}$ that we construct in this paper are the tip of the iceberg of a rich and interesting representation theory of this group and the double loop groups.

As for possible applications of this theory, note that the infinite Grassmannian and the fermionic Fock representation of $\widehat{G L}_{\infty}$ play an important role in the study of the $\mathrm{KP}$ hierarchy and closely related integrable hierarchies, such as KdV equations (see [DJKM, SW]). The gerbal representations of $G L_{\infty, \infty}$ which we construct here and their possible links to the 2-infinite Grassmannian may give us some clues as to what two-dimensional analogues of integrable hierarchies of soliton equations might look like. In particular, one can ask what is the two-dimensional analogue of the boson-fermion correspondence which is important in the study of the KP hierarchy.

The paper is organized as follows. In $₫$ we review the "1-dimensional story", that is, representations of the "master" group $G L_{\infty}$, from different points of view. We explain the construction of its central extension and describe a natural representation on a Fock module over an infinite-dimensional Clifford algebra. The goal of this paper is to develop an analogous representation theory for the "2-dimensional" group $G L_{\infty, \infty}$. The key difference is that representations of the latter are realized in categories rather than vector space. Hence we need to develop the formalism of actions of groups on categories. This is done in $\oint_{2}$. In particular, we show that to a gerbal representation of a group $G$ on an abelian category $\mathcal{C}$ corresponds a cohomology class in $H^{3}\left(G, \mathcal{Z}(\mathcal{C})^{\times}\right)$, where $\mathcal{Z}(\mathcal{C})$ is the center of $\mathcal{C}$.

In 93 we introduce the group $G L_{\infty, \infty}$ and related groups and Lie algebras. We construct a natural gerbal representation of $G L_{\infty, \infty}$ on an abelian category $\mathcal{C}_{\mathbb{L}}^{\text {ss }}$ of modules over a Clifford algebra. We show that it lifts to a genuine representation of the 2-group $\mathbb{G L}_{\infty, \infty}$ which is an extension of $G L_{\infty, \infty}$ by determinantal gerbes.

Next, we wish to calculte the third cohomology class corresponding to this gerbal representation of $G L_{\infty, \infty}$. We introduce the relevant cohomology groups in 4 . In particular, we discuss the universal $\mathbb{Z}$-central extension of $G L_{\infty, \infty}$, which is interesting on its own right. Computing the cohomology class of the gerbal representation $\mathcal{C}_{\mathbb{L}}^{\text {ss }}$ directly seems like a daunting, if not impossible, task. In \$5 we devise a different method. Namely, we develop the formalism of what we call "gerbal pairs of groups" which allows us to calculate in a regular way the third cohomology classes of gerbal representations in some situations. We then apply this formalism to a natural gerbal pair associated to the group $G L_{\infty, \infty}$. This alows us to calculate the desired cohomology class of the gerbal representation $\mathcal{C}_{\mathbb{L}}^{\text {ss }}$. At the end of $\$ 5$ we discuss representations of the group $\widehat{G L}_{\mathfrak{f}, \infty}$ on modules over a completion of the Clifford algebra $\mathrm{Cl}_{\mathcal{O}_{\mathbb{K}}}$, which may be of independent interest.

Acknowledgments. We thank D. Ben-Zvi, B. Feigin, D. Gaitsgory, V. Kac, D. Kazhdan and B. Tsygan for useful discussions. This paper was finished while E.F. visited Université Paris VI as Chaire d'Excellence of Fondation des Sciences Mathématiques 
de Paris. He thanks the Foundation for its support and the group "Algebraic Analysis" at Université Paris VI, and especially P. Schapira, for hospitality.

\section{1-Dimensional Story: Projective Representations}

In this section, we review the constructions of central extensions of the "master" group $G L_{\infty}$ and the Lie algebra $\mathfrak{g l}_{\infty}$ and their natural representations from different points of view. This objects are "1-dimensional" in the sense that they are attached to the algebra $\mathbb{C}((t))$ of formal Laurent power series in one variable. This section serves as a motivation of the main constructions of this paper concerning the "2-dimensional" case, when $\mathbb{C}((t))$ is replaced by power series in two variables. However, we will only consider the "2-dimensional" theory for groups in this paper and leave the Lie algebra case to [FZ].

1.1. Homological constructions. We first explain the construction for Lie algebras.

1.1.1. Tate vector spaces. Let $K=\mathbb{C}((t))$, regarded as a topological $\mathbb{C}$-vector space, with the usual $t$-adic topology. This is an example of the so-called Tate vector space.

We recall the following standard definitions (see, e.g., Kap ).

Definition 1.1. A topological vector space is called linearly compact if it is the topological dual of a discrete vector space. A topological space is called linearly locally compact, or Tate, if it admits a basis of neighborhoods of 0 consisting of linearly compact subspaces. A lattice in a Tate vector space $V$ is a linearly compact open subspace of $V$.

Any two lattices $L_{1}, L_{2}$ in a Tate vector space are commensurable with each other; that is, the quotients $L_{1} /\left(L_{1} \cap L_{2}\right)$ and $L_{2} /\left(L_{1} \cap L_{2}\right)$ are finite-dimensional.

Tate vector spaces form a category, whose Hom's are the continuous linear maps. This is an exact category in the sense of Quillen (see Q $\$ 2$ ). A sequence

$$
0 \rightarrow V^{\prime} \rightarrow V \rightarrow V^{\prime \prime} \rightarrow 0
$$

is an exact sequence in this category if it is an exact sequence of vector spaces, $V^{\prime} \rightarrow V$ is a closed embedding, and $V \rightarrow V^{\prime \prime}$ is an open surjective map. In the standard terminology of exact categories, $V^{\prime} \rightarrow V$ is called admissible monomorphism and denoted by $V^{\prime} \longmapsto V$, and $V \rightarrow V^{\prime \prime}$ is called admissible epimorphism and denoted by $V \rightarrow V^{\prime \prime}$. Note that in this case the topology on $V^{\prime \prime}$ coincides with the quotient topology.

Remark 1.2. We could identify the category of Tate vector spaces as a subcategory of pro-vector spaces by assigning $V$ the projective system $\left\{V / V_{\alpha}\right\}$, where $V_{\alpha}$ range over the set of open subspaces of $V$. Then a sequence $V^{\prime} \longmapsto V \rightarrow V^{\prime \prime}$ is exact if and only if there is an index set $I$ such that $V=\lim _{\alpha \in I} V_{\alpha}\left(\operatorname{resp} . V^{\prime}=\lim _{\alpha \in I} V_{\alpha}^{\prime} \cdot \operatorname{resp} . V^{\prime \prime}=\lim _{\alpha \in I} V_{\alpha}^{\prime \prime}\right)$, and for each $\alpha \in I$, the sequence $V_{\alpha}^{\prime} \rightarrow V_{\alpha} \rightarrow V_{\alpha}^{\prime \prime}$ is exact. Therefore, we see that if $V$ has a countable basis of neighborhood 0 , then any short exact sequence $V^{\prime} \longmapsto V \rightarrow V^{\prime \prime}$ splits.

We remark that the following discussion remains unchanged if we replace $K$ by any other non-compact Tate vector space. 
1.1.2. The Lie algebra $\mathfrak{g l}_{\infty}$. Let End $K$ be the algebra of continuous endomorphism of $K$ and $\mathfrak{g l}_{\infty}$ the associated Lie algebra. It is well-known that $H^{2}\left(\mathfrak{g l}_{\infty}\right)$ has a non-zero class $\left[c_{2}\right]$ and therefore there is a non-trivial $\mathbb{C}$-central extension of $\mathfrak{g l}_{\infty}$ corresponding to this class. Let us review the construction of this central extension following Kac-Peterson [KP and Arbarello-De Concini-Kac [ADK].

Recall that a lattice in $K$ is a compact open subspace of $K$. For example, $\mathcal{O}_{K}=$ $\mathbb{C}[[t]] \subset K$ is a lattice. Let $\mathfrak{g l}_{\infty}^{+}$be the Lie algebra of continuous endomorphisms of $\mathcal{O}_{K}$, and $\mathfrak{g l}_{\mathfrak{f}}$ the two-sided ideal of discrete endomorphisms, i.e., endomorphisms which have open kernels. Observe that there is a canonical trace functional $\operatorname{Tr}: \mathfrak{g l}_{\mathfrak{f}} \rightarrow \mathbb{C}$.

We write $K=\mathcal{O}_{K} \oplus \mathcal{O}^{-}$, where $\mathcal{O}^{-}$is a discrete vector space which could be chosen as $t^{-1} \mathbb{C}\left[t^{-1}\right]$, and denote by $\pi: K \rightarrow \mathcal{O}_{K}$ the projection onto the first factor. The projection induces a map $\mathfrak{g l}_{\infty} \rightarrow \mathfrak{g l}_{\infty}^{+}$, which is also denoted by $\pi$, as follows: for $g \in \mathfrak{g l}_{\infty}$, define $\pi(X) \in \mathfrak{g l}_{\infty}^{+}$by $\pi(X) v=\pi(X(v))$ for any $v \in \mathcal{O}_{K}$. Observe that $\pi: \mathfrak{g l}_{\infty} \rightarrow \mathfrak{g l}_{\infty}^{+}$is not a Lie algebra homomorphism.

We also introduce

$$
\widetilde{\mathfrak{g l}}_{\infty}=\left\{(A, X) \in \mathfrak{g l}_{\infty}^{+} \times \mathfrak{g l}_{\infty} \mid A-\pi(X) \in \mathfrak{g l}_{\mathfrak{f}}\right\}
$$

One then has the following exact sequence of associative algebras (and therefore Lie algebras)

$$
0 \rightarrow \mathfrak{g l}_{\mathfrak{f}} \stackrel{i}{\rightarrow} \widetilde{\mathfrak{g l}}_{\infty} \stackrel{p}{\rightarrow} \mathfrak{g l}_{\infty} \rightarrow 0
$$

where $i(A)=(A, 0)$ and $p(A, X)=X$. One also has a section of $p$ given by $X \mapsto$ $(\pi(X), X)$.

Since $\operatorname{Tr}: \mathfrak{g l}_{\mathfrak{f}} \rightarrow \mathbb{C}$ is a Lie algebra homomorphism, one can push out the exact sequence above to get a central extension of $\mathfrak{g l}_{\infty}$,

$$
0 \rightarrow \mathbb{C} \rightarrow \widehat{\mathfrak{g l}}_{\infty} \rightarrow \mathfrak{g l}_{\infty} \rightarrow 0
$$

It is clear that this central extension does not depend on the choice of a lattice $\mathcal{O}_{K}$ and the splitting $K=\mathcal{O}_{K} \oplus \mathcal{O}^{-}$. It is the universal central extension of $\mathfrak{g l}_{\infty}$. Observe that there is a spectral sequence of Lie algebra cohomology associated to (1.1), and the above construction shows that the class $\left[c_{2}\right] \in H^{2}\left(\mathfrak{g l}_{\infty}\right)$ corresponding to this central extension is obtained by $[\operatorname{Tr}] \in H^{1}\left(\mathfrak{g l}_{\mathfrak{f}}\right)$ by transgression.

1.1.3. The group $G L_{\infty}$. We will denote by $G L_{\infty}$ the group of continuous automorphisms of $K$. Then $\mathfrak{g l}_{\infty}$ could be viewed as the Lie algebra of $G L_{\infty}$. In fact, $G L_{\infty}$ is a group space (i.e., a sheaf of groups over $(\mathbf{A f f} / \mathbb{C})_{f p p f}$ ) which assigns to every commutative $\mathbb{C}$-algebra $R$ the group of continuous automorphisms of $R((t))$. We will return to this algebro-geometrical structure of $G L_{\infty}$ in 31.2 . For the moment, we just regard $G L_{\infty}$ as an abstract group.

Like in the case of Lie algebras, $H^{2}\left(G L_{\infty}, \mathbb{C}^{\times}\right)$has a non-trivial class which is obtained by transgression from $[\mathrm{det}] \in H^{1}\left(G L_{\mathfrak{f}}, \mathbb{C}^{\times}\right)$(what this means precisely is explained below). However, unlike the case of Lie algebra, it is difficult (and may be impossible) to write down the cocycle explicitly.

We will imitate the case of Lie algebras to define this central extension, as in [PS], Chapter 6 . So we let $G L_{\infty}^{+}$be the group of continuous automorphisms of $\mathcal{O}_{K}$ and $G L_{\mathfrak{f}}$ be the normal subgroup consisting of automorphisms fixing some open subspaces of $\mathcal{O}_{K}$. Remark that we have a canonical homomorphism det : $G L_{\mathfrak{f}} \rightarrow \mathbb{C}^{\times}$. Furthermore, let $\widetilde{G L}_{\infty}$ be the group of invertible elements in $\widetilde{\mathfrak{g l}}_{\infty}$ (regarded as an associative algebra). 
Unlike the case of Lie algebras, where we have a short exact sequence, here we only obtain a left exact sequence of groups

$$
1 \rightarrow G L_{\mathfrak{f}} \stackrel{i}{\rightarrow} \widetilde{G L}_{\infty} \stackrel{p}{\rightarrow} G L_{\infty},
$$

where $i: G L_{\mathfrak{f}} \rightarrow \widetilde{G L}_{\infty}$ is given by $i(a)=(a, 1)$. (We recall that $p(a, g)=g$.)

There is a surjective group homomorphism deg $: G L_{\infty} \rightarrow \mathbb{Z}$ defined as follows: for any $g \in G L_{\infty}$,

$$
\operatorname{deg}(g)=\operatorname{dim}\left(\frac{\mathcal{O}_{K}}{\mathcal{O}_{K} \cap g \mathcal{O}_{K}}\right)-\operatorname{dim}\left(\frac{g \mathcal{O}_{K}}{\mathcal{O}_{K} \cap g \mathcal{O}_{K}}\right)
$$

(see Proposition 4.3 for a more general discussion).

Denote $G L_{\infty}^{0}=\operatorname{ker}(\mathrm{deg})$. Then we have

$$
1 \rightarrow G L_{\mathfrak{f}} \rightarrow \widetilde{G L}_{\infty} \rightarrow G L_{\infty}^{0} \rightarrow 1
$$

(See Proposition 4.5 for the general case.) Pushing-out this sequence by det, we obtain a central extension of $G L_{\infty}^{0}$,

$$
1 \rightarrow \mathbb{C}^{\times} \rightarrow \widehat{G L}_{\infty}^{0} \rightarrow G L_{\infty}^{0} \rightarrow 1 .
$$

As in the case of Lie algebras, this central extension does not depend on the choice of the splitting. There is a spectral sequence associated to (1.3). According to the construction, the cohomology class in $H^{2}\left(G L_{\infty}^{0}, \mathbb{C}^{\times}\right)$corresponding to the central extension (1.4) comes by transgression from [det] $\in H^{1}\left(G L_{\mathfrak{f}}, \mathbb{C}^{\times}\right)$(which is non-trivial, see 4.2 .3$)$. We denote it by $\left[C_{2}^{0}\right]$ in what follows.

We would like to extend the above central extension to a central extension of $G L_{\infty}$.

Proposition 1.3. There is a unique (up to isomorphism) central extension

$$
1 \rightarrow \mathbb{C}^{\times} \rightarrow \widehat{G L}_{\infty} \rightarrow G L_{\infty} \rightarrow 1
$$

whose restriction to $G L_{\infty}^{0}$ is 1.4$)$.

Proof. Observe that $G L_{\infty}$ is the semi-direct product of $G L_{\infty}^{0}$ and $\mathbb{Z}$. Choose any element of degree one, e.g., $\sigma \in G L_{\infty}$ sending $t^{i} \mapsto t^{i+1}$ if we identify $K$ with $\mathbb{C}((t))$. Then $\langle\sigma\rangle \subset G L_{\infty}$ splits the map deg. The Lyndon-Hochschild-Serre spectral sequence implies that $H^{2}\left(G L_{\infty}, \mathbb{C}^{\times}\right) \cong H^{2}\left(G L_{\infty}^{0}, \mathbb{C}^{\times}\right)^{\langle\sigma\rangle}$, where $\sigma$ acts on $G L_{\infty}^{0}$ by conjugation. Indeed, observe that $H^{1}\left(G L_{\infty}^{0}, \mathbb{C}^{\times}\right)=0$ since there is an injection $H^{1}\left(G L_{\infty}^{0}, \mathbb{C}^{\times}\right) \rightarrow$ $H^{1}\left(\widetilde{G L}_{\infty}, \mathbb{C}^{\times}\right)$, and Proposition 4.2 claims that $H^{1}\left(\widetilde{G L}_{\infty}, \mathbb{C}^{\times}\right)=0$. On the other hand, $H^{i}\left(\mathbb{Z}, \mathbb{C}^{\times}\right)=0$ for $i \geq 2$.

We claim that the class $\left[C_{2}^{0}\right]$ is $\sigma$-invariant, hence in $H^{2}\left(G L_{\infty}^{0}, \mathbb{C}^{\times}\right)^{\langle\sigma\rangle}$, and therefore the proposition follows.

To prove this, we only need to give an automorphism $\widehat{\sigma}: \widehat{G L}_{\infty}^{0} \rightarrow \widehat{G L}_{\infty}^{0}$ lifting the automorphism $\sigma: G L_{\infty}^{0} \rightarrow G L_{\infty}^{0}, b \rightarrow \sigma b \sigma^{-1}$. In fact, we will give a group homomorphism $\tilde{\sigma}: \widetilde{G L}_{\infty} \rightarrow \widetilde{G L}_{\infty}$ which lifts $\sigma$.

Namely, we choose a splitting $\mathcal{O}_{K}=\sigma \mathcal{O}_{K} \oplus \mathbb{C}$. For any $a \in G L_{\infty}^{+}$, let $a_{\sigma}=\sigma a \sigma^{-1} \oplus$ id $: \sigma \mathcal{O}_{K} \oplus \mathbb{C} \rightarrow \sigma \mathcal{O}_{K} \oplus \mathbb{C}$. Then set $\tilde{\sigma}(a, g)=\left(a_{\sigma}, \sigma g \sigma^{-1}\right)$.

It is clear that $\tilde{\sigma}$ is an injective homomorphism $\widetilde{G L}_{\infty} \rightarrow \widetilde{G L}_{\infty}$ and gives an injective homomorphism $\widehat{\sigma}: \widehat{G L}_{\infty}^{0} \rightarrow \widehat{G L}_{\infty}^{0}$. Although $\tilde{\sigma}$ is not a group automorphism (since it is not surjective), $\widehat{\sigma}$ is surjective and gives a group automorphism of $\widehat{G L}_{\infty}^{0}$ covering $\sigma$. This is because for any $a \in G L_{\infty}^{+}$one can find $e, e^{\prime} \in G L_{\mathfrak{f}}$, $\operatorname{det}(e)=\operatorname{det}\left(e^{\prime}\right)=1$ such that $e a e^{\prime}=a_{\sigma}^{\prime}$ for some $a^{\prime} \in G L_{\infty}^{+}$(by means of elementary transformations). Therefore, 
any element in $\widehat{G L}_{\infty}^{0}$ may be represented by an element $\left(a_{\sigma}, b\right)=\tilde{\sigma}\left(a, \sigma^{-1} b \sigma\right) \in \widetilde{G L}_{\infty}$. This completes the proof that $\left[C_{2}^{0}\right] \in H^{2}\left(G L_{\infty}^{0}, \mathbb{C}^{\times}\right)^{\langle\sigma\rangle}$.

The corresponding central extension (1.5) is just obtained by forming the semi-direct product $\widehat{G L}_{\infty}=\widehat{G L}_{\infty}^{0} \rtimes\langle\sigma\rangle$, where $\sigma$ acts on $\widehat{G L}_{\infty}^{0}$ via $\widehat{\sigma}$ constructed above.

Remark 1.4. One can show that there is no automorphism $\tilde{\sigma}: \widetilde{G L}_{\infty} \rightarrow \widetilde{G L}_{\infty}$ covering $\sigma$. Therefore, there is no extension of $G L_{\infty}$ by $G L$ whose restriction to $G L_{\infty}^{0}$ gives (1.3).

Observe that while the automorphism group of the sequence (1.4) is trivial, the automorphism group of (1.5) is $H^{1}\left(G L_{\infty}, \mathbb{C}^{\times}\right)=\mathbb{C}^{\times}$.

1.2. Geometric constructions: non-linear version. In the previous section we have constructed central extensions of $G L_{\infty}$ and $\mathfrak{g l}_{\infty}$ corresponding to given cohomology classes by homological methods. While the homological construction tells us where the cohomology classes come from, it will be more meaningful if we could see such central extensions come into life more naturally. We will justify the "naturality" in this section from the geometric point of view and in the next section from the algebraic point of view.

1.2.1. The infinite Grassmannian. Recall that $K$ is a Tate vector space. The infinite Grassmannian (i.e. the Sato Grassmannian) is defined as the moduli space of lattices in $K$. More precisely, for any commutative $\mathbb{C}$-algebra $R$, we set

$$
\operatorname{Gr}(R)=\left\{\begin{array}{c}
L \subset R \widehat{\otimes} K \text { a } R \text {-submodule, } t^{N}\left(R \widehat{\otimes} \mathcal{O}_{K}\right) \subset L \subset t^{-N}\left(R \widehat{\otimes} \mathcal{O}_{K}\right), \\
\text { for some } N \in \mathbb{Z}_{+}, L / t^{N}\left(R \widehat{\otimes} \mathcal{O}_{K}\right) \text { is a projective } R \text {-module }
\end{array}\right\} .
$$

We show in $\$ 1.2 .2$ below that the corresponding functor is represented by an indscheme over $\mathbb{C}$. Denote by $L_{0}$ the standard lattice $\mathcal{O}_{K} \subset K$. The virtual dimension of $L$ is defined as

$$
\operatorname{dim} L=\operatorname{dim} \frac{L}{L \cap L_{0}}-\operatorname{dim} \frac{L_{0}}{L \cap L_{0}} .
$$

Then the connected components of Gr are labeled by the virtual dimensions. Namely, we set

$$
\operatorname{Gr}^{n}(\mathbb{C})=\{L \in \operatorname{Gr}(\mathbb{C}), \operatorname{dim} L=n\}
$$

Then

$$
\mathrm{Gr}=\bigsqcup_{n \in \mathbb{Z}} \mathrm{Gr}^{n}
$$

The group $G L_{\infty}$ acts on $\operatorname{Gr}(\mathbb{C})$ by sending $L$ to $g L$ for any $g \in G L_{\infty}$. This action is transitive. (More generally, the group space $G L_{\infty}$ acts on $\mathrm{Gr}$, and $\mathrm{Gr}$ is a homogeneous space of $G L_{\infty}$.) It follows from the definition that $G L_{\infty}^{0}$ fixes each component, and $\sigma$ sends $\mathrm{Gr}^{n}$ to $\mathrm{Gr}^{n-1}$. Similarly, $\mathfrak{g l}_{\infty}$ also acts on $\mathrm{Gr}$, i.e., each element $X \in \mathfrak{g l}_{\infty}$ gives rise a vector field on Gr.

A great discovery of the Sato school was that the action of an infinite-dimensional abelian Lie subalgebra of $\mathfrak{g l}_{\infty}$ gives rise to the KP hierarchy DJKM, SW, which explains the importance of the infinite Grassmannian in soliton theory. 
1.2.2. The determinant line bundle. Let $R$ be a commutative $\mathbb{C}$-algebra. Given $L \in$ $\operatorname{Gr}^{n}(R)$, one could find $N \in \mathbb{Z}_{+}$such that $t^{N}\left(R \widehat{\otimes} \mathcal{O}_{K}\right) \subset L \subset t^{-N}\left(R \widehat{\otimes} \mathcal{O}_{K}\right)$. Then $M=L / t^{N}\left(R \widehat{\otimes} \mathcal{O}_{K}\right)$ defines a projective $R$-module of rank $(N+n)$ in

$$
R^{2 N} \cong t^{-N}\left(R \widehat{\otimes} \mathcal{O}_{K}\right) / t^{N}\left(R \widehat{\otimes} \mathcal{O}_{K}\right)
$$

This way, we obtain that

$$
\mathrm{Gr}^{n}=\lim _{\vec{N}} G(N+n, 2 N),
$$

where $G(k, n)$ is the usual Grassmannian of $k$-planes in the $n$-dimensional vector space. The closed embedding $G(N+n, 2 N) \rightarrow G(N+n+1,2 N+2)$ is obtained by sending $M \subset R^{2 N}$ to $M \oplus R \subset R^{2 N+2}$. This gives $\mathrm{Gr}^{n}$ the structure of an ind-scheme.

The Grassmannian $G(k, n)$ carries a determinant line bundle, which assigns to each $k$-plane the line of its top exterior power. This is the negative generator of the Picard group of $G(k, n)$ (recall that $\operatorname{Pic}(G(k, n) \cong \mathbb{Z})$. Under the embedding $G(N+n, 2 N) \rightarrow$ $G(N+n+1,2 N+2)$, the determinant line bundle on $G(N+n+1,2 N+2)$ restricts to the determinant line bundle on $G(N+n, 2 N)$. Therefore, $\operatorname{Pic}\left(\mathrm{Gr}^{n}\right) \cong \mathbb{Z}$ and there is a determinant line bundle on $\mathrm{Gr}^{n}$, which is the negative generator of its Picard group, and restricts to the determinant line bundle on each $G(N+n, 2 N)$. We denote by $\mathcal{L}$ the line bundle on $\mathrm{Gr}$ that restricts to the determinant line bundle on each connected component. We call $\mathcal{L}$ the determinant line bundle on $\mathrm{Gr}$.

More invariantly, if $L, L^{\prime} \in \operatorname{Gr}(R)$ are two lattices, then there is a positive integer $N$ such that $L, L^{\prime} \supset t^{N}\left(R \widehat{\otimes} \mathcal{O}_{K}\right)$ and both $L /\left(R \widehat{\otimes} \mathcal{O}_{K}\right), L^{\prime} /\left(R \widehat{\otimes} \mathcal{O}_{K}\right)$ are projective. Then one defines an invertible $R$-module by

$$
\operatorname{det}\left(L \mid L^{\prime}\right):=\bigwedge^{\mathrm{top}}\left(L / t^{N}\left(R \widehat{\otimes} \mathcal{O}_{K}\right)\right) \otimes\left(\bigwedge^{\mathrm{top}}\left(L^{\prime} / t^{N}\left(R \widehat{\otimes} \mathcal{O}_{K}\right)\right)^{-1} .\right.
$$

It is clear that this $R$-module is independent of the choice of $N$ up to a canonical isomorphism. Therefore, we obtain a line bundle over $\mathrm{Gr} \times \mathrm{Gr}$. If we identify $\mathrm{Gr} \cong$ Gr $\times\left\{L_{0}\right\} \hookrightarrow \mathrm{Gr} \times \mathrm{Gr}$, the above line bundle restricts to the determinant line bundle on Gr.

Remark 1.5. The important feature of the determinant lines is that for $L, L^{\prime}, L^{\prime \prime} \in$ $\operatorname{Gr}(R)$, there is a canonical isomorphism

$$
\gamma_{L, L^{\prime}, L^{\prime \prime}}: \operatorname{det}\left(L \mid L^{\prime}\right) \otimes \operatorname{det}\left(L^{\prime} \mid L^{\prime \prime}\right) \cong \operatorname{det}\left(L \mid L^{\prime \prime}\right)
$$

such that for any $L, L^{\prime}, L^{\prime \prime}, L^{\prime \prime \prime} \in \operatorname{Gr}(R), \gamma_{L, L^{\prime}, L^{\prime \prime \prime}} \gamma_{L^{\prime}, L^{\prime \prime}, L^{\prime \prime \prime}}=\gamma_{L, L^{\prime \prime}, L^{\prime \prime \prime}} \gamma_{L, L^{\prime}, L^{\prime \prime}}$.

In what follows, $\operatorname{det}\left(L \mid L^{\prime}\right)^{\times}$will denote the set of nowhere vanishing sections of $\operatorname{det}\left(L \mid L^{\prime}\right)$, regarded as a line bundle on $\operatorname{Spec} R$.

1.2.3. Central extensions. Now, we regard $G L_{\infty}$ as a group space. Let $\mathcal{L}$ be the determinant line bundle on Gr. Since $\operatorname{Pic}\left(\mathrm{Gr}^{n}\right) \cong \mathbb{Z}$, for any $g \in G L_{\infty}(\mathbb{C})$, we have $g^{*} \mathcal{L} \cong \mathcal{L}$. So it is natural to ask whether the action of $G L_{\infty}(\mathbb{C})$ on $\operatorname{Gr}(\mathbb{C})$ could be lifted to an action on $\mathcal{L}$. That is, whether we can choose isomorphisms $i_{g}: g^{*} \mathcal{L} \cong \mathcal{L}$, such that $i_{g h}=i_{h} \circ h^{*}\left(i_{g}\right):(g h)^{*} \mathcal{L} \cong \mathcal{L}$. Unfortunately (or fortunately!), this is not the case.

There is a canonical $\mathbb{G}_{m}$-central extension $\widehat{G L}_{\infty}^{\prime}$ of $G L_{\infty}$ which tautologically acts on $\mathcal{L}$ (see, e.g., $\mathrm{Kap}$ ). The $R$-points of $\widehat{G L}_{\infty}^{\prime}$ are pairs $(c, g)$ with $g \in G L_{\infty}(R)$ and $c \in \operatorname{det}\left(g L_{0} \mid L_{0}\right)^{\times}$. The multiplication is $(c, g)\left(c^{\prime}, g^{\prime}\right)=\left(c g\left(c^{\prime}\right), g g^{\prime}\right)$. Here $g(c) \in$ $\operatorname{det}\left(g g^{\prime} L_{0} \mid g L_{0}\right)^{\times}$, and therefore by (1.7)

$$
c g\left(c^{\prime}\right) \in \operatorname{det}\left(g L_{0} \mid L_{0}\right)^{\times} \otimes\left(g g^{\prime} L_{0} \mid g L_{0}\right)^{\times} \cong \operatorname{det}\left(g g^{\prime} L_{0} \mid L_{0}\right)^{\times} .
$$


Furthermore, Remark 1.5 guarantees that the group structure of $\widehat{G L}_{\infty}^{\prime}$ is well-defined.

The natural action of $\widehat{G L}_{\infty}^{\prime}$ on $\mathcal{L}$ is defined as follows. We observe that the diagonal action of $G L_{\infty}$ on $\mathrm{Gr} \times \mathrm{Gr}$ lifts to an action on the line bundle over it simply via the canonical identification

$$
\operatorname{det}\left(L \mid L^{\prime}\right)=\operatorname{det}\left(g L \mid g L^{\prime}\right) .
$$

Observe that the fiber of $\mathcal{L}$ over $L \in \operatorname{Gr}(R)$ is $\mathcal{L}_{L}=\operatorname{det}\left(L \mid L_{0}\right)$. Therefore, for given $(c, g) \in \widehat{G L}_{\infty}^{\prime}(R)$,

$$
\mathcal{L}_{L}=\operatorname{det}\left(L \mid L_{0}\right)=\operatorname{det}\left(g L \mid g L_{0}\right) \stackrel{\otimes c}{\rightarrow} \operatorname{det}\left(g L \mid g L_{0}\right) \otimes \operatorname{det}\left(g L_{0} \mid L_{0}\right) \cong \operatorname{det}\left(g L \mid L_{0}\right)=\mathcal{L}_{g L}
$$

defines an action of $\widehat{G L}_{\infty}^{\prime}$ on $\mathcal{L}$.

Proposition 1.6. The group of $\mathbb{C}$-points of the central extension $\widehat{G L}_{\infty}^{\prime}$ constructed above is isomorphic to the extension (1.5).

Proof. By Proposition 1.3, it is enough to prove that the above central extension, when restricted to $G L_{\infty}^{0}$, is isomorphic to (1.4). Recall the definition of $\widetilde{G L}_{\infty}$. We will show that there is a homomorphism $\widetilde{G L}_{\infty} \rightarrow \widehat{G L}_{\infty}^{\prime}$ sending $(a, g) \in \widetilde{G L}_{\infty}$ to $(c, g) \in \widehat{G L}_{\infty}^{\prime}$, where $g \in G L_{\infty}^{0}, a \in G L_{\infty}^{+}$and $c \in \operatorname{det}\left(g L_{0} \mid L_{0}\right)^{\times}$, such that if $(a, g)=(a, 1) \in G L_{\mathfrak{f}}$ and $\operatorname{det}(a)=1$, then $c=1$. It is clear that this implies that the "neutral" component of $\widehat{G L}_{\infty}^{\prime}(\mathbb{C})$ is obtained by pushing-out $\widetilde{G L}_{\infty}$ via det and therefore is isomorphic to (1.4).

Indeed, regard $a \in G L_{\infty}^{+}$as a continuous automorphism of $L_{0}=\mathcal{O}_{K}$. We know that there is a sublattice $L \subset L_{0} \cap g^{-1} L_{0}$ such that $\left.a\right|_{L}=\left.\pi(g)\right|_{L}$ since $a-\pi(g) \in \mathfrak{g l}_{\mathfrak{f}}$. Since $g(L) \subset L_{0},\left.\pi(g)\right|_{L}=\left.g\right|_{L}$. Therefore $a(L)=g(L)$ is a lattice in $L_{0}$. Then we obtain the following isomorphism

$$
\frac{g L_{0}}{g(L)} \stackrel{g^{-1}}{\longrightarrow} \frac{L_{0}}{L} \stackrel{a}{\longrightarrow} \frac{L_{0}}{a(L)}=\frac{L_{0}}{g(L)} .
$$

The top exterior power of the above isomorphism gives us the desired element

$$
c \in \operatorname{Hom}\left(\bigwedge^{\operatorname{top}}\left(g\left(L_{0}\right) / g(L)\right), \bigwedge^{\operatorname{top}}\left(L_{0} / g(L)\right)\right) \cong \operatorname{det}\left(g L_{0} \mid L_{0}\right)^{\times},
$$

and it is clear that if $g=1$ (then $a \in G L_{\mathfrak{f}}$ ), then $c$ constructed above is just $\operatorname{det}(a) \in$ $\operatorname{det}\left(L_{0} \mid L_{0}\right)^{\times}=\mathbb{C}^{\times}$. This completes the proof of the proposition.

So in what follows we will not distinguish between $\widehat{G L}_{\infty}$ and $\widehat{G L}_{\infty}^{\prime}$.

1.2.4. The gerbe of determinantal theories. We rephrase the above construction, using the determinantal gerbe $\mathcal{D}_{K}$ associated to the Tate vector space $K$. We first recall some basic facts about the determinantal gerbe associated to a Tate vector space here for reader's convenience. The following definition is due to M. Kapranov Kap.

Definition 1.7. Let $V$ be a Tate vector space. A determinantal theory $\Delta$ on $V$ is a rule that assigns to every lattice $L \subset V$ a line $\Delta(L)$ and to every $L_{1} \subset L_{2}$ an isomorphism

$$
\Delta_{L_{1}, L_{2}}: \Delta\left(L_{1}\right) \otimes \operatorname{det}\left(L_{2} / L_{1}\right) \rightarrow \Delta\left(L_{2}\right)
$$

such that for any $L_{1} \subset L_{2} \subset L_{3}$ three lattices, the obvious diagram

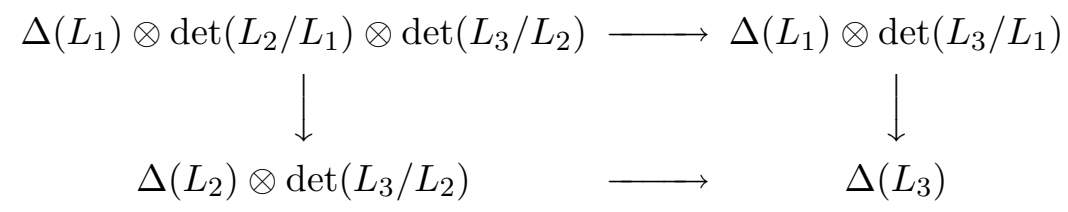


is commutative. The category whose objects are determinantal theories on $V$ and morphisms are isomorphisms of determinantal theories (defined in the obvious way) is a $\mathbb{C}^{\times}$-gerbe, called the determinantal gerbe of $V$ and denoted by $\mathcal{D}_{V}$.

There is another $\mathbb{C}^{\times}$-gerbe that is described in $\mathrm{ADK}$. Namely, objects are lattices in $V$ and $\operatorname{Hom}\left(L, L^{\prime}\right)=\operatorname{det}\left(L \mid L^{\prime}\right)^{\times}$. It is clear that the latter gerbe is equivalent to the former under the functor that sends a lattice $L \subset V$ to the determinantal theory $\Delta$ which assigns $\Delta(L)=\mathbb{C}$ (this assignment uniquely determines $\Delta$ ).

The following lemma is the content of [Kap, Proposition 1.4.5, and [AK], Lemma 7. Let $A$ be an abelian group. Recall that if $\mathcal{F}, \mathcal{F}^{\prime}$ are two $A$-gerbes, the tensor product $\mathcal{F} \otimes \mathcal{F}^{\prime}$ is also an $A$-gerbe, defined as follows. The objects in $\mathcal{F} \otimes \mathcal{F}^{\prime}$ are $\left(x, x^{\prime}\right)$ with $x$ an object in $\mathcal{F}$ and $x^{\prime}$ an object in $\mathcal{F}^{\prime}$. The morphisms between $\left(x, x^{\prime}\right)$ and $\left(y, y^{\prime}\right)$ are

$$
\operatorname{Hom}_{\mathcal{F} \otimes \mathcal{F}^{\prime}}\left(\left(x, x^{\prime}\right),\left(y, y^{\prime}\right)\right):=\operatorname{Hom}_{\mathcal{F}}(x, y) \otimes_{A} \operatorname{Hom}_{\mathcal{F}^{\prime}}\left(x^{\prime}, y^{\prime}\right) .
$$

Lemma 1.8. Let $V^{\prime} \longmapsto V$ be an admissible monomorphism. Then there is a canonical equivalence of $\mathbb{C}^{\times}$-gerbes

$$
\mathcal{D}_{V^{\prime}} \otimes \mathcal{D}_{V / V^{\prime}} \cong \mathcal{D}_{V}
$$

and for $V_{1} \longmapsto V_{2} \longmapsto V_{3}$ we have a natural transformation

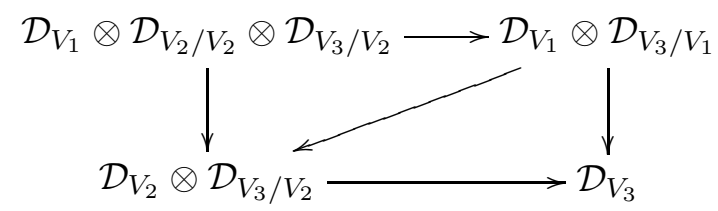

such that if given $V_{1} \longmapsto V_{2} \longmapsto V_{3} \longmapsto V_{4}$, the cubical diagram of natural transformations commutes.

Observe that if $\varphi: V \rightarrow V^{\prime}$ is an isomorphism, we obtain a natural equivalence of categories $\mathcal{D}_{\varphi}: \mathcal{D}_{V} \cong \mathcal{D}_{V^{\prime}}$.

It would be possible to define $\mathcal{D}_{K}$ as a sheaf of groupoids over $\mathbf{A f f} / \mathbb{C}$ with appropriate topology, if one could make sense of a family of Tate vector spaces (or Tate modules). This has been done by $\mathrm{V}$. Drinfeld in $[\mathrm{Dr}$, and it is a non-trivial fact from loc. cit. that the determinantal gerbe of a Tate $R$-module is a $\mathbb{G}_{m}$-gerbe over $\operatorname{Spec} R$ in Nisnevich topology (see loc. cit. $\S 3.6$ and $\S 5.2$ ).

Now, the group $G L_{\infty}$ acts on the gerbe $\mathcal{D}_{K}$. Moreover, there is a $G L_{\infty}$-equivariant covering $\mathrm{Gr} \rightarrow \mathcal{D}_{K}$. At the level of $\mathbb{C}$-points, this map sends $L \in \operatorname{Gr}(\mathbb{C})$ to the determinantal theory $\Delta$ which assigns $\Delta(L)=\mathbb{C}$. Recall that we denote by $\mathcal{L}$ the determinant line bundle on $\operatorname{Gr} \times \operatorname{Gr}$. We will denote by $\operatorname{Tot}(\mathcal{L})^{\times}$its total space with zero section deleted. Then

$$
\operatorname{Gr} \times \mathcal{D}_{K} \operatorname{Gr} \cong \operatorname{Tot}(\mathcal{L})^{\times}
$$

In fact, we can recover the central extension of $G L_{\infty}$ just from $\mathcal{D}_{K}$. Given a map $L: \operatorname{Spec} \mathbb{C} \rightarrow \mathcal{D}_{K}$, we obtain a morphism act $t_{L} G L_{\infty} \rightarrow \mathcal{D}_{K}$. By [AK], Theorem 1, which goes back to Brylinski,

$$
\widehat{G L}_{\infty, L}:=\operatorname{Spec} \mathbb{C} \times_{\mathcal{D}_{K}} G L_{\infty} \rightarrow G L_{\infty}
$$

is a $\mathbb{G}_{m}$-central extension of $G L_{\infty}$. In particular, if $L=L_{0}, \widehat{G L}_{\infty, L_{0}}=\widehat{G L}_{\infty}$ is the central extension we constructed in previous subsection. 
1.3. Algebraic constructions: linear version. At this point, it would be desirable to produce a representation of the central extensions of $G L_{\infty}$ and $\mathfrak{g l}_{\infty}$ constructed above, on which the central elements act non-trivially. This is the fermionic Fock representation introduced in $[\mathrm{KP}$.

1.3.1. The space of global sections of the determinant line bundle. Recall that the determinant line bundle $\mathcal{L}$ on $\mathrm{Gr}$ is $\widehat{G L}_{\infty}$-equivariant. We define

$$
\bigwedge:=\Gamma\left(\mathrm{Gr}, \mathcal{L}^{*}\right)^{*}
$$

This is a representation of $\widehat{G L}_{\infty}$, on which the central subgroup $\mathbb{C}^{\times}$acts by the identity. We now give a more concrete description of $\bigwedge$.

1.3.2. Clifford modules. Let $K=\mathbb{C}((t))$. This is a topological vector space (with the usual $t$-adic topology) whose topological dual is (defined to be) $K^{*}=\mathbb{C}((t)) d t$. There is a natural symmetric bilinear form on $K \oplus K^{*}$ induced by the residue pairing. Let $\mathrm{Cl}_{K}=\mathrm{Cl}\left(K \oplus K^{*}\right)$ be the corresponding completed topological Clifford algebra. If we set $\phi_{n}=t^{n}$ and $\phi_{n}^{*}=t^{n} \frac{d t}{t}$ (note that in this notation, the dual of $\phi_{n}$ is $\left.\phi_{-n}^{*} !\right)$, then the algebra is topologically generated by $\phi_{n}, \phi_{m}^{*}$ subject to the following relations

$$
\left[\phi_{n}, \phi_{m}\right]_{+}=0, \quad\left[\phi_{n}^{*}, \phi_{m}^{*}\right]_{+}=0, \quad\left[\phi_{n}, \phi_{m}^{*}\right]_{+}=\delta_{n,-m} .
$$

Since $\mathcal{O}_{K} \oplus \mathcal{O}_{K} d t$ is a Lagrangian subspace of $K \oplus K^{*}, \bigwedge\left(\mathcal{O}_{K} \oplus \mathcal{O}_{K} d t\right)$ is a subalgebra of $\mathrm{Cl}_{K}$. Let Ind $\mathrm{Cl}_{\left(\mathcal{O}_{K} \oplus \mathcal{O}_{K} d t\right)}(\mathbb{C}|0\rangle)$ be the $\mathrm{Cl}_{K}$-module generated be the (vacuum) vector $|0\rangle_{\mathcal{O}_{K}}$, on which $\bigwedge\left(\mathcal{O}_{K} \oplus \mathcal{O}_{K} d t\right)$ acts by 0 . This is a discrete $\mathrm{Cl}_{K}$-module, called the fermionic Fock module. The following lemma can be proved by reducing to the finitedimensional case.

Lemma 1.9. $\operatorname{Ind}_{\bigwedge\left(\mathcal{O}_{K} \oplus \mathcal{O}_{K} d t\right)}^{\mathrm{Cl}_{K}}\left(\mathbb{C}|0\rangle_{\mathcal{O}_{K}}\right) \cong \Gamma\left(\mathrm{Gr}, \mathcal{L}^{*}\right)^{*}=\bigwedge$.

Thus, $\operatorname{Ind}_{\bigwedge(\mathcal{O} \oplus \mathcal{O} d t)}^{\mathrm{Cl}_{K}}(\mathbb{C}|0\rangle)$ is a concrete realization of the representation $\bigwedge$ of $\widehat{G L}_{\infty}$.

1.3.3. Another description of the action of $\widehat{G L}_{\infty}$ on $\bigwedge$. We may also discover this representation of $\widehat{G L}_{\infty}$ from the representation-theoretic point of view. The starting point is, as in the finite-dimensional case, the following statement:

Lemma 1.10. The category of discrete $\mathrm{Cl}_{K}$-modules $\mathcal{C}_{K}$ is equivalent to the category of vector spaces. Any non-zero discrete irreducible $\mathrm{Cl}_{K}$-module is isomorphic to $\bigwedge$.

Proof. Consider the functor $F$ from the category $\mathcal{C}_{K}$ to the category of vector spaces taking a $\mathrm{Cl}_{K}$-module $M$ to the space of invariants of the subalgebra $\bigwedge\left(\mathcal{O}_{K} \oplus \mathcal{O}_{K} d t\right) \subset$ $\mathrm{Cl}_{K}$ in $M$. Let $G$ be the left adjoint functor which sends a vector space $V$ to $V \otimes \bigwedge$, where $\mathrm{Cl}_{K}$ acts on the second factor. It is easy to see that the space of $\wedge\left(\mathcal{O}_{K} \oplus \mathcal{O}_{K} d t\right)$ invariants in $\Lambda$ is spanned by the vacuum vector $|0\rangle_{\mathcal{O}_{K}}$. For this we identify $\Lambda$ with $\bigwedge\left(t^{-1} \mathbb{C}\left[t^{-1}\right] \oplus t^{-1} \mathbb{C}\left[t^{-1}\right] d t\right)$ and construct a basis of monomials in the generators $\phi_{n}, n<$ 0 , and $\phi_{n}^{*}, n \leq 0$. One readily checks that a non-constant linear combination of such monomials cannot possibly be annihilated by $\phi_{m}, m \geq 0$, and $\phi_{m}^{*}, m>0$. This shows that $F \circ G$ is the identity functor. Let us show that $G \circ F$ is also isomorphic to the identity functor.

Thus, we have a homomorphism $F(M) \otimes \wedge \rightarrow M$ and we have to prove that it is an isomorphism. First of all, $\Lambda$ is an irreducible $\mathrm{Cl}_{K}$-module. To see that, we use the above explicit realization to give $\wedge$ a $\mathbb{Z}$-grading such that $\operatorname{deg} \phi_{n}=\operatorname{deg} \phi_{n}^{*}=-n$. 
Any non-zero submodule is graded. This implies that it must be generated by a vector that is invariant under $\bigwedge\left(\mathcal{O}_{K} \oplus \mathcal{O}_{K} d t\right) \subset \mathrm{Cl}_{K}$, hence by $|0\rangle_{\mathcal{O}_{K}}$. This implies that the map $F(M) \otimes \wedge \rightarrow M$ is injective. Next, let us show that if $M \neq 0$, then $F(M) \neq 0$. To see that, observe that for any vector $v$ in a discrete $\mathrm{Cl}_{K}$-module $M$ there exists $N \in \mathbb{Z}_{+}$such that $\bigwedge\left(t^{N+1} \mathcal{O}_{K} \oplus t^{N} \mathcal{O}_{K} d t\right) \cdot v=0$. Consider the vector space $V_{N}=$ $t^{-N} \mathcal{O}_{K} / t^{N+1} \mathcal{O}_{K}$ and its dual vector space $V_{N}^{*} \simeq t^{-N} \mathcal{O}_{K} \frac{d t}{t} / t^{N+1} \mathcal{O}_{K} \frac{d t}{t}$. Let $\mathrm{Cl}_{V_{N}}$ be the Clifford algebra associated to $V_{N} \oplus V_{N}^{*}$. Then $\mathrm{Cl}_{V_{N}} \cdot v \subset \mathrm{Cl}_{K} \cdot v$. It follows from the theory of modules over finite-dimensional Clifford algebras that the former has a non-zero vector invariant under $\bigwedge\left(\mathcal{O}_{K} / t^{N+1} \mathcal{O}_{K} \oplus \mathcal{O}_{K} d t / t^{N} \mathcal{O}_{K} d t\right)$. Viewed as a vector of $M$, it is then $\bigwedge\left(\mathcal{O}_{K} \oplus \mathcal{O}_{K} d t\right)$-invariant, and so $F(M) \neq 0$. Now suppose that $F(M) \otimes \wedge \rightarrow M$ is not surjective, and $M^{\prime} \neq 0$ is the cokernel. Then $M^{\prime}$ contains a non-zero $\wedge\left(\mathcal{O}_{K} \oplus \mathcal{O}_{K} d t\right)$-invariant vector. Reducing to finite-dimensional Clifford algebras, as above, we obtain that it can be lifted to a $\bigwedge\left(\mathcal{O}_{K} \oplus \mathcal{O}_{K} d t\right)$-invariant vector in $M$ itself. This is a contradiction, which completes the proof.

Thus, the category of $\mathrm{Cl}_{K}$-modules is very simple. On the other hand, we can construct many "natural" non-trivial discrete irreducible $\mathrm{Cl}_{K}$-modules. Namely, for any $L \in \operatorname{Gr}(\mathbb{C}), L \oplus L^{\perp}$ is a Lagrangian in $K \oplus K^{*}$ and therefore

$$
M_{L}:=\operatorname{Ind}_{\bigwedge\left(L \oplus L^{\perp}\right)}^{\mathrm{Cl}_{K}}\left(\mathbb{C}|0\rangle_{L}\right)
$$

is a discrete irreducible $\mathrm{Cl}_{K}$-module. Therefore, all of them are isomorphic to $M_{L_{0}}=\bigwedge$. We have the following simple observation.

Lemma 1.11. For any $L, L^{\prime} \in \operatorname{Gr}(\mathbb{C})$, there is a canonical isomorphism

$$
\operatorname{Hom}\left(M_{L}, M_{L^{\prime}}\right) \cong \operatorname{det}\left(L \mid L^{\prime}\right) \text {. }
$$

such that for $L, L^{\prime}, L^{\prime \prime}$, the following diagram commutes.

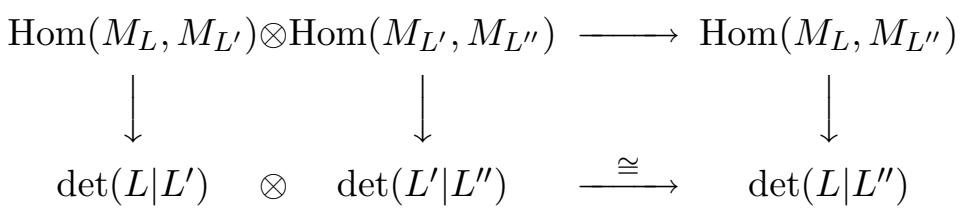

Proof. By Schur's lemma, $\operatorname{Hom}\left(M_{L}, M_{L^{\prime}}\right)$ is one-dimensional. The statement of the lemma identifies this one-dimensional space canonically.

Since $M_{L}$ is generated by a vector $|0\rangle_{L}$ annihilated by the subalgebra $\bigwedge\left(L \oplus L^{\perp}\right)$, defining a homomorphism $M_{L} \rightarrow M_{L^{\prime}}$ is equivalent to choosing a $\bigwedge\left(L \oplus L^{\perp}\right)$-invariant vector in $M_{L^{\prime}}$. Thus, $\operatorname{Hom}\left(M_{L}, M_{L^{\prime}}\right)$ is canonically identified with the space of $\bigwedge(L \oplus$ $L^{\perp}$ )-invariants in $M_{L^{\prime}}$. The latter module is, in turn, generated by a vector $|0\rangle_{L^{\prime}}$ annihilated by the subalgebra $\bigwedge\left(L^{\prime} \oplus L^{\prime \perp}\right)$.

Now, there is a positive integer $N$ such that

$$
t^{N} \mathcal{O}_{K} \subset L, L^{\prime} \subset t^{-N} \mathcal{O}_{K}, \quad t^{N} \mathcal{O}_{K} \frac{d t}{t} \subset L^{\perp}, L^{\prime \perp} \subset t^{-N} \mathcal{O}_{K} \frac{d t}{t} .
$$

We have the Clifford algebra $\mathrm{Cl}_{V_{N}}$ introduced in the proof of Lemma 1.10 and for any subspace $U \subset V_{N}$ its irreducible module $M_{U}$ defined in the same way as above. It is clear that the space of $\bigwedge\left(L \oplus L^{\perp}\right)$-invariants in $M_{L^{\prime}}$ is equal to the space of $\bigwedge\left(\left(L / t^{N} \mathcal{O}_{K}\right) \oplus\left(L / t^{N} \mathcal{O}_{K}\right)^{\perp}\right)$-invariants in $M_{L^{\prime} / t^{N} \mathcal{O}_{K}}$. The latter space is canonically identified with $\operatorname{det}\left(L \mid L^{\prime}\right)$ (see formula (1.6) $)$. 
More explicitly, these homomorphisms may be constructed as follows. Suppose for simplicity that $L^{\prime} \subset L$. Let us choose a basis $\left\{v_{i}\right\}_{i \in I}$, of $L / L^{\prime}$. Choose a lifting $\widetilde{v}_{i}$ of $v_{i}$ to $L \subset K$. Then the vector

$$
\wedge_{i \in I} \widetilde{v}_{i}|0\rangle_{L^{\prime}} \in M_{L^{\prime}}
$$

is independent of the choice of the liftings and is annihilated by $\bigwedge\left(L \oplus L^{\perp}\right)$. All homomorphisms $M_{L} \rightarrow M_{L^{\prime}}$ are defined by sending $|0\rangle_{L} \subset M_{L}$ to a multiple of this vector, and we have a canonical identification of $\operatorname{Hom}\left(M_{L}, M_{L^{\prime}}\right)$ with $\operatorname{det}\left(L / L^{\prime}\right)=$ $\operatorname{det}\left(L \mid L^{\prime}\right)$.

Now we define an action of $\widehat{G L}_{\infty}$ on $\bigwedge$. Since $G L_{\infty}$ acts on $K$ via continuous automorphisms, it acts on $\mathrm{Cl}_{K}$ via continuous automorphisms as well. However, this action does not necessarily lift to an action of any given $\mathrm{Cl}_{K}$-module. Rather, what we have is an action of $G L_{\infty}$ on the the category of discrete $\mathrm{Cl}_{K}$-modules (see Example 2.7 for more details). Explicitly, for any $g \in G L_{\infty}$ and any $\mathrm{Cl}_{K}$-module $M$, we obtain a new $\mathrm{Cl}_{K}$-module $M^{g}$, whose underlying vector space is the same as $M$, but for any $a \in \mathrm{Cl}_{K}, m \in M^{g}$, the action is given by the formula $a \cdot m=g^{-1}(a) m$. Clearly then, $\bigwedge^{g}=M_{g L_{0}}$. This module is isomorphic to $\bigwedge$. However, there is no canonical isomorphism, and choosing particular isomorphisms for different $g$ we necessarily obtain a projective action of $G L_{\infty}$.

More precisely, we obtain a representation of $\widehat{G L}_{\infty}$ by the formula

$$
(c, g): \bigwedge \stackrel{\text { id }}{\rightarrow} \bigwedge^{g} \stackrel{c}{\rightarrow} \bigwedge
$$

Here, id : $\bigwedge \rightarrow \bigwedge^{g}$ is the identity map as vector spaces, and

$$
c \in \operatorname{Hom}\left(\bigwedge^{g}, \bigwedge\right)^{\times}=\operatorname{Hom}\left(M_{g L_{0}}, M_{L_{0}}\right)^{\times}=\operatorname{det}\left(g L_{0} \mid L_{0}\right)^{\times} .
$$

Recalling the definition of the central extension $\widehat{G L}_{\infty}^{\prime}=\widehat{G L}_{\infty}$ given in 1.2 .3 , we see this representation of $\widehat{G L}_{\infty}$ on $\Lambda$ is the same as the one we have constructed in the previous section.

\section{Generalities on the actions of groups on CAtegories}

In the previous section we have given a brief account of the 1-dimensional story. We explained how to construct representations of the central extensions of the 1dimensional "master" Lie algebra $\mathfrak{g l}_{\infty}$ and the corresponding group $G L_{\infty}$, both algebraically and geometrically. Algebraically, they are realized by using Fock representations of a Clifford algebra. Geometrically, this representation may be obtained by using the determinant line bundle on the infinite Grassmannian.

Now we begin the study of the 2-dimensional story. In the next section we will define the corresponding "master" Lie algebra $\mathfrak{g l}_{\infty, \infty}$ and the group $G L_{\infty, \infty}$. Our goal is to construct an abelian category on which $\mathfrak{g l}_{\infty, \infty}$ and $G L_{\infty, \infty}$ act gerbally, realizing their non-trivial third cohomology classes. (We will calculate the corresponding cohomology class of $G L_{\infty, \infty}$ in $\$ 5.3$.)

In this section we develop the necessary general formalism of group actions on categories. There is a similar theory for gerbal actions of Lie algebras on abelian categories, but it involves more sophisticated machinery. Roughly speaking, we need to develop the theory of gerbal actions of groups in "families", that is, over arbitrary bases. We may then define a gerbal action of a Lie algebra as the action of the corresponding 
formal group. This material will be discussed in the follow-up paper [FZ] where we will also give examples of gerbal representations of the Lie algebra $\mathfrak{g l}_{\infty, \infty}$.

\subsection{2-groups.}

2.1.1. Definition of 2-groups. We recall the definition of 2-groups. A good introduction for this subject is $\mathrm{BL}$.

Definition 2.1. A 2-group is a monoidal groupoid $\mathcal{G}$ such that the set of isomorphism classes of objects of $\mathcal{G}$, denoted by $\pi_{0}(\mathcal{G})$, is a group under the multiplication induced from the monoidal structure. Let $I$ denote the unit object of $\mathcal{G}$. We set $\pi_{1}(\mathcal{G})=\operatorname{End}_{\mathcal{G}} I$.

In the literature, these objects often appear under different names. For example, they are called weak 2-groups in $\mathrm{BL}$, and are called gr-categories in [S].

It is clear that any group (in the usual sense) can be regarded as a 2-group with the trivial $\pi_{1}$. All 2-groups form a (strict) 2-category, with objects being 2-groups, 1-morphism being the homomorphisms between 2-groups (i.e. monoidal functors), and 2-morphism being the monoidal natural transformations of monoidal functors.

We recall that if the monoidal structure of a 2-group is upgraded to a tensor category structure (i.e., there exists a commutativity constraint whose square is the identity), then this 2-group is called a Picard groupoid. Therefore, Picard groupoids should be regarded as commutative 2 -groups.

It is easy to prove that $\pi_{1}(\mathcal{G})$ is always an abelian group. The following simple observation will be important to us.

Lemma 2.2. Given a 2-group $\mathcal{G}$, there is a natural action of $\pi_{0}(\mathcal{G})$ on $\pi_{1}(\mathcal{G})$ by automorphisms, i.e., there is a natural group homomorphism $\pi_{0}(\mathcal{G}) \rightarrow \operatorname{Aut}\left(\pi_{1}(\mathcal{G})\right)$.

Proof. Denote $G=\pi_{0}(\mathcal{G})$ and $A=\pi_{1}(\mathcal{G})$. Observe that for any object $x$ of $\mathcal{G}$, $\operatorname{End}_{\mathcal{G}}(x)$ is isomorphic to $A$ in two ways. Namely, $l_{x}: A \rightarrow \operatorname{End}_{\mathcal{G}}(x)$ is obtained by the canonical isomorphism $I \otimes x \cong x$, and $r_{x}: A \rightarrow \operatorname{End}_{\mathcal{G}}(x)$ by $x \otimes I \cong x$. Therefore, for any $x$, we define $\rho_{x}:=l_{x}^{-1} \circ r_{x}: A \rightarrow A$. By definition, we know that for any $a \in A, \rho_{x}(a)$ is the unique element in $A$ such that the following diagram is commutative:

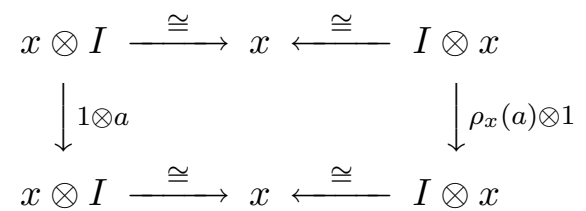

Its uniqueness implies the following two properties which complete the proof of the lemma.

(i) If $x \cong x^{\prime}$, then $\rho_{x}=\rho_{x^{\prime}}$;

(ii) $\rho_{I}=$ Id and $\rho_{x} \rho_{x^{\prime}}=\rho_{x \otimes x^{\prime}}$.

Remark 2.3. (i) The above construction is functorial. Let $F: \mathcal{G} \rightarrow \mathcal{H}$ be a homomorphism of 2-groups. Then it induces group homomorphisms $F_{0}: \pi_{0}(\mathcal{G}) \rightarrow \pi_{0}(\mathcal{H})$ and $F_{1}: \pi_{1}(\mathcal{G}) \rightarrow \pi_{1}(\mathcal{H})$. We have $F\left(\rho_{x}(a)\right)=\rho_{F(x)}(F(a))$.

(ii) It is known (in particular, from the unpublished thesis of Grothendieck's student Sinh [S], see also [BL]) that 2-groups with $\pi_{0}=G$ and $\pi_{1}=A$ are classified by $H^{3}(G, A)$ (the so-called Postnikov invariant). The above lemma is the first step toward the construction of a class in $H^{3}(G, A)$ associated to $\mathcal{G}$. Although we will not use this statement in this paper (so we do not make it precise), our construction will be closely related to this statement, but from a different point of view. 
(iii) As usual, we could work in any topos $\mathcal{T}$ instead of the category of sets. Then a sheaf of 2-group $\mathcal{G}$ will be a stack, such that for any $U \in \mathcal{T}, \mathcal{G}(U)$ is a 2-group and the pullback functor respects to the monoidal structure (i.e. for $f: V \rightarrow U, g: W \rightarrow V$, $f^{*}, g^{*}$ are monoidal functors, and the canonical isomorphism $g^{*} \circ f^{*} \cong(f \circ g)^{*}$ are monoidal natural transforms). Denote by $I_{U}$ the unit object in $\mathcal{G}(U)$. Observe that $U \mapsto \operatorname{End}_{\mathcal{G}(U)}\left(I_{U}\right)$ is a sheaf of abelian groups over $\mathcal{T}$, which is denoted by $\pi_{1}(\mathcal{G})$. However, $U \mapsto \pi_{0}(\mathcal{G}(U))$ is usually only a presheaf. We will denote its sheafification by $\pi_{0}(\mathcal{G})$ (so in general $\pi_{0}(\mathcal{G})(U) \neq \pi_{0}(\mathcal{G}(U))$ ). This is a sheaf of groups, called the coarse moduli of $\mathcal{G}$. Remark (i) shows that there is an action of $\pi_{0}(\mathcal{G})$ on $\pi_{1}(\mathcal{G})$. If one regards $\pi_{0}(\mathcal{G})$ as a 2-group, then the natural projection $\pi: \mathcal{G} \rightarrow \pi_{0}(\mathcal{G})$ is a 2-group homomorphism.

2.2. Central extensions. We will discuss a special type of 2-groups which can be regarded as the central extensions of groups by Picard groupoids. We will confine ourselves to the situations that are needed in the following. For a much more general treatment of extensions of groups by 2 -groups, see $[\mathrm{Br}$. We will work in the topos of sets.

Let $\mathcal{P}$ be a Picard groupoid. Recall that a $\mathcal{P}$-torsor $\mathcal{Q}$ (over a point) is a module category over $\mathcal{P}$, i.e., there is a bifunctor $\otimes: \mathcal{P} \times \mathcal{Q} \rightarrow \mathcal{Q}$ satisfying the associativity constraint, such that the functor $I \otimes$ - is isomorphic to the identity functor and for any $x \in \mathcal{Q}$, the functor $\cdot \otimes x: \mathcal{P} \rightarrow \mathcal{Q}$ is an equivalence of categories.

Let $A$ be an abelian group. Then it makes perfect sense to tensor two $A$-torsors over $A$, which is again an $A$-torsor. This tensor product makes the category of $A$ torsors a Picard groupoid, denoted by $B A$. It is clear that a $B A$-torsor in the standard terminology is just an $A$-gerbe.

We will call $\mathcal{Q}$ a $\mathcal{P}$-bitorsor if it is equipped with two commuting $\mathcal{P}$-torsor structures. See $[\mathrm{Br}$, Definition 3.1.8 for the more general definition of bi-torsors of a 2-group. The meaning of "commuting $\mathcal{P}$-torsor structures" is spelled out in (3.1.8.2)-(3.1.8.4) of loc. cit..

Now let $\mathcal{G}$ be a 2-group. Denote $A=\pi_{1}(\mathcal{G})=\operatorname{End}_{\mathcal{G}}(I)$. We let $\mathcal{G}_{e}$ be the subgroupoid of objects in $\mathcal{G}$ that are isomorphic to $I$. Therefore, $\mathcal{G}_{e}$ is a connected groupoid, and the functor $x \mapsto \operatorname{Hom}_{\mathcal{G}}(I, x), \mathcal{G}_{e} \rightarrow B A$ is an equivalence of tensor categories. Now for any $s \in \pi_{0}(\mathcal{G})$, let $\mathcal{G}_{s}$ be the subgroupoid consisting of objects in the isomorphism class $s$. Then the monoidal functor gives $\mathcal{G}_{s}$ the structure of a bi-torsor under $\mathcal{G}_{e}$. Namely, $l: \mathcal{G}_{e} \otimes \mathcal{G}_{s} \rightarrow \mathcal{G}_{s}$ and $r: \mathcal{G}_{s} \otimes \mathcal{G}_{e} \rightarrow \mathcal{G}_{s}$.

Lemma 2.4. The following statements are equivalent:

(1) The action of $\pi_{0}(\mathcal{G})$ on $\pi_{1}(\mathcal{G})$ is trivial;

(2) The monoidal functor $\mathcal{G}_{e} \rightarrow \mathcal{G}$ is central in the sense of [Be, Definition 1;

(3) The two A-gerbe structures on $\mathcal{G}_{s}$ are the same.

Definition 2.5. If a 2-group $\mathcal{G}$ satisfies the above conditions, then we call it the central extension of $G=\pi_{0}(\mathcal{G})$ by $B A$.

2.3. Actions of groups on categories. We now begin to discuss actions of groups on categories. Our approach in this section will be naive in the sense that we will work mostly with groups of $\mathbb{C}$-points of algebraic groups. In [FZ] we will develop the theory in a way that will allow us to work in families and take full advantage of the algebrogeometric structures. This will allow us to introduce the notion of a Lie algebra action on a category which will be important for our purposes. 
2.3.1. The center. We will use the following notation. If $\lambda: F \rightarrow G$ is a morphism between two functors $F$ and $G$ acting from a category $\mathcal{C}$ to a category $\mathcal{C}^{\prime}$, we denote $\lambda_{X}: F(X) \rightarrow G(X)$ the specialization of $\lambda$ to $X$.

Now let $\mathcal{C}$ be an abelian category. The center $\mathcal{Z}(\mathcal{C})$ of $\mathcal{C}$ is by definition the ring $\operatorname{End} \mathbf{1}_{\mathcal{C}}$, where $\mathbf{1}_{\mathcal{C}}$ is the identity functor of $\mathcal{C}$. Thus, an element $a \in \mathcal{Z}(\mathcal{C})$ assigns to every $X \in \mathcal{C}$ a morphism $a_{X} \in \operatorname{End}_{\mathcal{C}} X$ such that for any $f: X \rightarrow Y, a_{Y} \circ f=f \circ a_{X}$. It is easy to see that $\mathcal{Z}(\mathcal{C})$ is in fact a commutative ring. For instance, if $\mathcal{C}=A-\operatorname{Mod}$, the category of left-modules over a ring $A$, then $\mathcal{Z}(\mathcal{C})=Z(A)$, the center of $A$. For any $X, Y \in \mathcal{C}, \operatorname{Hom}_{\mathcal{C}}(X, Y)$ is a $\mathcal{Z}(\mathcal{C})$-module.

Let $\mathcal{Z}(\mathcal{C})^{\times}$be the group of invertible elements in $\mathcal{Z}(\mathcal{C})$. This is an abelian group which may be defined for any category as the automorphism group of the identity functor.

2.3.2. The 2-group $\mathbb{G L}(\mathcal{C})$. Let $\mathcal{C}$ be a $\mathbb{C}$-linear abelian category. We denote by $\mathbb{G L}(\mathcal{C})$ the category of $\mathbb{C}$-linear auto-equivalences of $\mathcal{C}$. By definition, the objects of $\mathbb{G} \mathbb{L}(\mathcal{C})$ are $\mathbb{C}$-linear additive functors $F: \mathcal{C} \rightarrow \mathcal{C}$ which are equivalences of categories. The morphisms $\operatorname{Hom}_{\mathbb{G L}(\mathcal{C})}(F, G)$ are the natural transformations from $F$ to $G$ which are isomorphisms. It is clear from the definition that $\mathbb{G L}(\mathcal{C})$ is a strict monoidal category. Furthermore, $\mathbb{G} \mathbb{L}(\mathcal{C})$ is a 2-group, with $\pi_{1}(\mathbb{G L}(\mathcal{C}))=\mathcal{Z}(\mathcal{C})^{\times}$. (We will prove in [FZ] that $\mathbb{G} \mathbb{L}(\mathcal{C})$ may be regarded as the groupoid of $\mathbb{C}$-points of a stack.)

Likewise, for any category $\mathcal{C}$, not necessarily abelian, we can define the 2-group $\mathbb{A} U \mathbb{T}(\mathcal{C})$ of auto-equivalences of $\mathcal{C}$, whose objects are all auto-equivalences of $\mathcal{C}$ and morphisms are isomorphisms between auto-equivalences. However, this 2-group does not possess rich structure.

\subsubsection{Genuine actions. Let $\mathcal{G}$ be a 2-group.}

Definition 2.6. A (genuine) representation of $\mathcal{G}$ on an abelian category $\mathcal{C}$ is a homomorphism of 2-groups $F: \mathcal{G} \rightarrow \mathbb{G} \mathbb{L}(\mathcal{C})$. Likewise, an action of $\mathcal{G}$ on a general category is a homomorphism of 2-groups $\mathcal{G} \rightarrow \mathbb{A U T}(\mathcal{C})$.

If $\mathcal{G}=G$ is just an ordinary group, then the above definition gives us the usual notion of action of a group on categories (see the Introduction).

Example 2.7. Here is a basic example. Assume that $G$ acts on a $\mathbb{C}$-algebra $R$ by automorphisms. Then it acts on the category of (left) $R$-modules in the following way. Let $m: R \otimes M \rightarrow M$ be a left $R$-module. Then define a new $R$-module structure on $M$ by the formula

$$
\left(F_{g}(m)(r, x)=m\left(g^{-1} r, x\right) \text { for } r \in R, x \in M .\right.
$$

If $f:(\rho, M) \rightarrow\left(\rho^{\prime}, M^{\prime}\right)$ is a morphism between $R$-modules, then define $F_{g}(f)=f$ as linear map between underlying $\mathbb{C}$-vector spaces. Obviously, $F_{g}: \mathcal{C} \rightarrow \mathcal{C}$ is a functor. It is easy to check that $F$ defines an action of $G$ on $\mathcal{C}$.

The genuine actions of $\mathcal{G}$ on $\mathcal{C}$ form a category. Namely, $\operatorname{Hom}_{2-\mathrm{Grp}}(\mathcal{G}, \mathbb{A} \mathbb{U T}(\mathcal{C}))$. (We recall that all 2-groups form a strict 2-category 2-Grp.)

2.3.4. Gerbal actions. A genuine representation of a group on a category is a categorical analogue of a representation of a group on a vector space. We also want some categorical analogue of the projective representations, which will be called gerbal representations. We will only consider gerbal representations of groups in this paper. In [FZ] we will 
develop the theory further and introduce the notion of a gerbal representation of a Lie algebra.

Definition 2.8. A gerbal representation of a group $G$ on an abelian category $\mathcal{C}$ is a homomorphism of groups $F: G \rightarrow \pi_{0}(\mathbb{G L}(\mathcal{C}))$. Likewise, a gerbal action of $G$ on a general category $\mathcal{C}$ is a group homomorphism $G \rightarrow \pi_{0}(\mathbb{A U T}(\mathcal{C}))$.

Equivalently, this is an assignment to each $g \in G$ of an auto-equivalence $F_{g}: \mathcal{C} \rightarrow \mathcal{C}$ such that $F_{e} \cong \mathbf{1}_{\mathcal{C}}$ and $F_{g} F_{g^{\prime}} \cong F_{g g^{\prime}}$.

A homomorphism of gerbal representations $\mathcal{C}$ and $\mathcal{C}^{\prime}$ of $G$ is a functor $H: \mathcal{C} \rightarrow \mathcal{C}^{\prime}$ such that there exist isomorphisms $H \circ F_{g} \simeq F_{g} \circ H$ for all $g \in G$. If $H$ is an equivalence of categories, we call these representations equivalent.

Remark 2.9. One can give another definition, in which one also specifies, as part of the data, isomorphisms between $F_{g} F_{g^{\prime}}$ and $F_{g g^{\prime}}$ for all $g, g^{\prime} \in G$. We have discussed this definition in the Introduction. The two definitions are essentially equivalent. As an analogy, consider the notion of projective representation of a group $G$ on a $\mathbb{C}$-vector space. This may be defined as a homomorphism $G \rightarrow \operatorname{PGL}(V)$ or as a rule that assigns to each $g \in G$ an automorphism $T_{g}$ of $V$ such that $T_{g} T_{h}=\alpha_{g, h} T_{g h}$, for some $\alpha_{g, h} \in \mathbb{C}^{\times}$. The above definition of gerbal representation is an analogue of the former, whereas the definition used in the Introduction is an analogue of the latter. We find the above definition more convenient and economical, because it avoids the data of the isomorphisms which are in some sense redundant, as we will see below.

Given a gerbal action of $G$ on $\mathcal{C}$, one obtains, by Lemma 2.2, an action of $G$ on $\pi_{1}(\mathbb{G} \mathbb{L}(\mathcal{C}))=\mathcal{Z}(\mathcal{C})^{\times}$. More explicitly, the homomorphism $\rho: G \rightarrow \operatorname{Aut}\left(\mathcal{Z}(\mathcal{C})^{\times}\right)$is defined as follows: for any $g \in G$ and $a \in \mathcal{Z}(\mathcal{C})^{\times}$,

$$
\rho_{g}(a)_{F_{g}(X)}=F_{g}\left(a_{X}\right)
$$

for any object $X$ in $\mathcal{C}$. (It is easy to see that this condition determines $\rho$ uniquely.) Observe that if $\mathcal{C}$ is abelian and this is a gerbal representation, the above formula in fact defines an action of $G$ on $\mathcal{Z}(\mathcal{C})$.

We also observe that for any two objects $x, y$ in $\mathbb{G} \mathbb{L}(\mathcal{C})$ (or in $\mathbb{A U T}(\mathcal{C})$ ), $\operatorname{Hom}(x, y)$ is a bi-pseudotorsor ${ }^{3}$ under $\mathcal{Z}(\mathcal{C})^{\times}$. Indeed, $\operatorname{Hom}_{\mathcal{G}}(x, y)$ is a pseudo $\operatorname{End}(x)$-torsor under the left action and a pseudo $\operatorname{End}(y)$-torsor under the right action. A prior, we obtain four pseudo $\mathcal{Z}(\mathcal{C})^{\times}$-torsor structures on $\operatorname{Hom}(x, y)$ since there are two isomorphisms $l_{x}, r_{x}: \mathcal{Z}(\mathcal{C})^{\times} \rightarrow \operatorname{End}(x)$ and two isomorphisms $l_{y}, r_{y}: \mathcal{Z}(\mathcal{C})^{\times} \rightarrow \operatorname{End}(y)$. However, it is clear that the two pseudo $\mathcal{Z}(\mathcal{C})^{\times}$-torsor structures obtained by $l_{x}$ and $l_{y}$ in fact coincide. So do the other two. Therefore, there are only two pseudo $\mathcal{Z}(\mathcal{C})^{\times}$-torsor structures on $\operatorname{Hom}(x, y)$, which furthermore commute with each other. (These are the two $\mathcal{Z}(\mathcal{C})^{\times}$-gerbe structures on $\mathbb{G} \mathbb{L}(\mathcal{C})$ (and on $\mathbb{A U T}(\mathcal{C})$ ).) In what follows, we will use the pseudo $\mathcal{Z}(\mathcal{C})^{\times}$-torsor structure on $\operatorname{Hom}(x, y)$ coming from $l_{x}$.

2.3.5. Third cohomology class. Let us pick an isomorphism $c\left(g_{1}, g_{2}\right): F_{g_{1}} F_{g_{2}} \stackrel{\cong}{\longrightarrow} F_{g_{1} g_{2}}$ for all pairs $g_{1}, g_{2} \in G$. Then for $g_{1}, g_{2}, g_{3} \in G$, there are two isomorphisms between $F_{g_{1}} F_{g_{2}} F_{g_{3}}$ and $F_{g_{1} g_{2} g_{3}}$; namely,

$$
F_{g_{1}} F_{g_{2}} F_{g_{3}} \stackrel{\cong}{\longrightarrow} F_{g_{1} g_{2}} F_{g_{3}} \stackrel{\cong}{\longrightarrow} F_{g_{1} g_{2} g_{3}}
$$

\footnotetext{
${ }^{3}$ We recall that, for a group $G$, a $G$-pseudotorsor is a set that is either empty or is a torsor under $G$.
} 
and

$$
F_{g_{1}} F_{g_{2}} F_{g_{3}} \stackrel{\cong}{\longrightarrow} F_{g_{1}} F_{g_{2} g_{3}} \stackrel{\cong}{\longrightarrow} F_{g_{1} g_{2} g_{3}}
$$

Let $a\left(g_{1}, g_{2}, g_{3}\right) \in \mathcal{Z}(\mathcal{C})^{\times}$be the unique element sending the first isomorphism to the second one since $\operatorname{Hom}\left(F_{g_{1}} F_{g_{2}} F_{g_{3}}, F_{g_{1} g_{2} g_{3}}\right)$ is a torsor under $\mathcal{Z}(\mathcal{C})^{\times}$. That is, the following diagram is commutative:

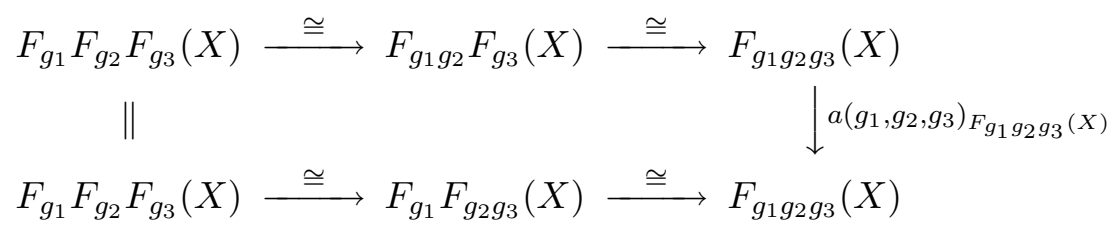

In this way, one defines a map $a: G \times G \times G \rightarrow \mathcal{Z}(\mathcal{C})^{\times}$.

Theorem 2.10. (i) a is a cocycle, i.e.

$$
\rho_{g_{1}} a\left(g_{2}, g_{3}, g_{4}\right) a\left(g_{1}, g_{2} g_{3}, g_{4}\right) a\left(g_{1}, g_{2}, g_{3}\right)=a\left(g_{1} g_{2}, g_{3}, g_{4}\right) a\left(g_{1}, g_{2}, g_{3} g_{4}\right)
$$

(ii) Given different isomorphisms $c^{\prime}\left(g_{1}, g_{2}\right): F_{g_{1}} F_{g_{2}} \stackrel{\cong}{\longrightarrow} F_{g_{1} g_{2}}$, the new cocycle differs from the original one by a coboundary, and therefore, there is a well-defined cohomology class $[a] \in H^{3}\left(G, \mathcal{Z}(\mathcal{C})^{\times}\right)$associated to a gerbal action of the group $G$ on the category $\mathcal{C}$.

(iii) If this class is trivial, then the action $F$ could be upgraded to a genuine action, i.e., one could choose $c\left(g_{1}, g_{2}\right): F_{g_{1}} F_{g_{2}} \stackrel{\cong}{\longrightarrow} F_{g_{1} g_{2}}$ in such a way that the following diagram is commutative:

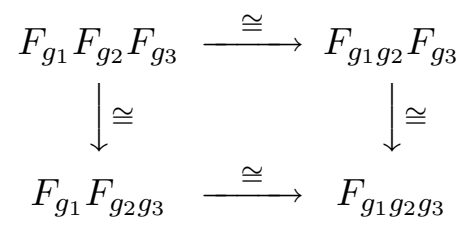

In this case, the set of isomorphisms $c\left(g_{1}, g_{2}\right)$ such that $F$ is a genuine action is a torsor under $H^{2}\left(G, \mathcal{Z}(\mathcal{C})^{\times}\right)$. Furthermore, the automorphism of such $F$ is $H^{1}\left(G, \mathcal{Z}(\mathcal{C})^{\times}\right)$.

Remark 2.11. (i) There is a tautological gerbal action of the group $\pi_{0}(\mathbb{A} \mathbb{U} \mathbb{T}(\mathcal{C}))$ on $\mathcal{C}$, which gives a cohomology class $u \in H^{3}\left(\pi_{0}(\mathbb{A U T}(\mathcal{C})), \mathcal{Z}(\mathcal{C})^{\times}\right)$. This is the Postnikov invariant associated to the 2-group $\mathbb{A U T}(\mathcal{C})$. Then the cohomology class $[a]$ in the theorem is just the pullback of $u$.

(ii) Part (iii) of the theorem could be interpreted as follows: if the class $[a]$ vanishes, then the group homomorphism $F: G \rightarrow \pi_{0}(\mathbb{A U T}(\mathcal{C}))$ can be lifted as a 2group homomorphism $\tilde{F}: G \rightarrow \mathbb{A} \mathbb{U T}(\mathcal{C})$. The possible liftings form a subcategory of $\operatorname{Hom}_{2-\operatorname{Grp}}(G, \mathbb{A} \mathbb{U T}(\mathcal{C}))$. The isomorphism classes form a torsor under $H^{2}\left(G, \mathcal{Z}(\mathcal{C})^{\times}\right)$. Given a lifting $\tilde{F}$, the automorphism group of $\tilde{F}$ (i.e., invertible 2-morphisms between $\tilde{F}$ and itself $)$ is $H^{1}\left(G, \mathcal{Z}(\mathcal{C})^{\times}\right)$.

(iii) The same remarks apply to $\mathbb{G} \mathbb{L}(\mathcal{C})$.

Proof. (i) is proved by diagram chasing. Observe that the following diagram is always commutative: 


$$
\begin{array}{rrr}
F_{g_{1}} F_{g_{2}} F_{g_{3}} F_{g_{4}} & \frac{F_{g_{1}} F_{g_{2}}\left(c\left(g_{3}, g_{4}\right)\right)}{\cong} F_{g_{1}} F_{g_{2}} F_{g_{3} g_{4}} \\
\cong \downarrow c\left(g_{1}, c_{2}\right) & \cong \downarrow c\left(g_{1}, g_{2}\right) \\
F_{g_{1} g_{2}} F_{g_{3}} F_{g_{4}} & \frac{F_{g_{1} g_{2}}\left(c\left(g_{3}, g_{4}\right)\right)}{\cong} & F_{g_{1} g_{2}} F_{g_{3} g_{4}}
\end{array}
$$

To prove (i), it is enough to show that both sides are the same when evaluated at $F_{g_{1} g_{2} g_{3} g_{4}}(X)$, for any object $X$ in $\mathcal{C}$. That is, to show that the rightmost loop in the following diagram commutes. This follows from the fact that all other loops in the diagram are commutative.

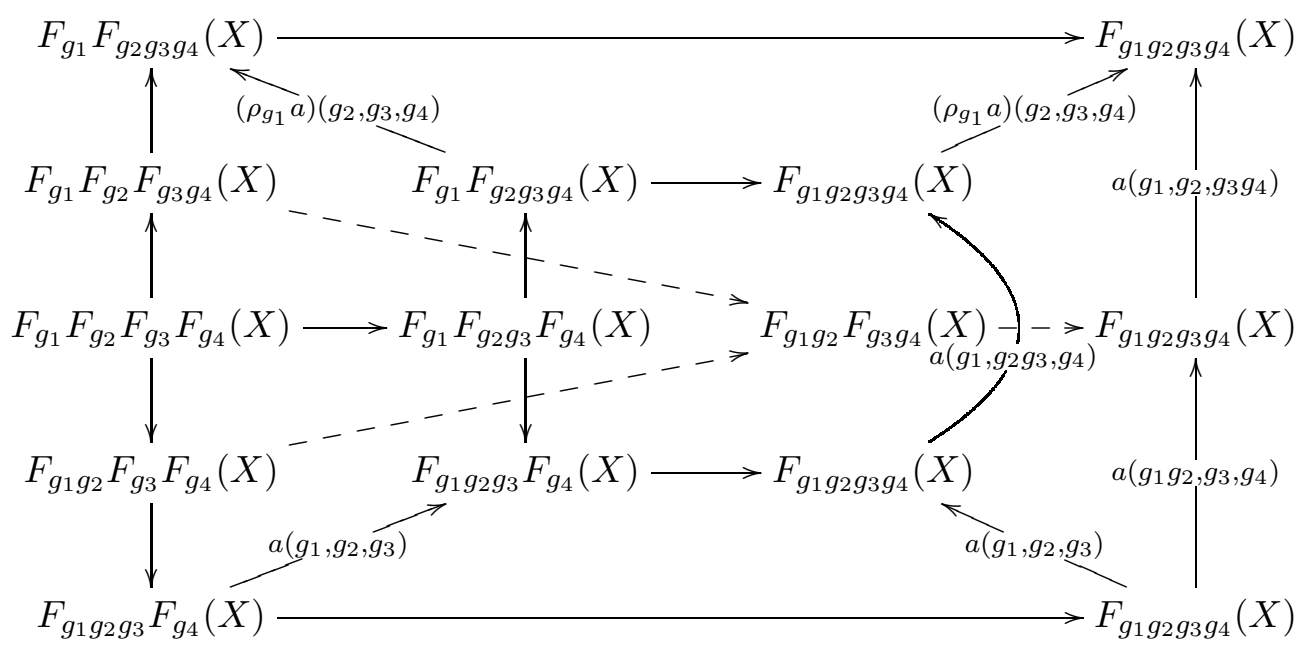

For (ii), observe that we could write $c^{\prime}\left(g_{1}, g_{2}\right)=c\left(g_{1}, g_{2}\right) \cdot d\left(g_{1}, g_{2}\right)$ for a unique $d\left(g_{1}, g_{2}\right) \in Z(\mathcal{C})^{\times}$. Then it is easy to see that $a^{\prime}=a+\delta d$. (iii) is standard.

Thus, we associate to a gerbal action of a group $G$ on a category $\mathcal{C}$ a third cohomology class of $G$ with coefficients in $\mathcal{Z}(\mathcal{C})^{\times}$, which is the obstruction to upgrade the gerbal action to a genuine action.

We will apply the following simple observation in $\$ 5.3$, Let $\mathcal{D} \subset \mathcal{C}$ be a subcategory of $\mathcal{C}$. We call $\mathcal{D}$ is invariant under the gerbal action $F$ of $G$ if for any $u: X \rightarrow Y \in \mathcal{D}$, $F_{g}(u): F_{g}(X) \rightarrow F_{g}(Y) \in \mathcal{D}$ for any $g \in G$. Then we have a gerbal action of $G$ on $\mathcal{D}$. Observe that there is a natural $G$-module homomorphism $r: \mathcal{Z}(\mathcal{C})^{\times} \rightarrow \mathcal{Z}(\mathcal{D})^{\times}$by restriction. We have the following

Proposition 2.12. Let $a \in H^{3}\left(G, \mathcal{Z}(\mathcal{C})^{\times}\right)$be the obstruction to upgrade the gerbal action of $G$ on $\mathcal{C}$ to a genuine action. Then the obstruction in $H^{3}\left(G, \mathcal{Z}(\mathcal{D})^{\times}\right)$to upgrade the gerbal action of $G$ on $\mathcal{D}$ is $r(a)$.

2.3.6. An example. Here is an example of the gerbal action of a group on a category.

Let $R$ be an associative $\mathbb{C}$-algebra, and $\mathcal{C}$ be the category of left $R$-modules. We assume that the $G$ acts on $R$ via outer automorphisms, that is we have a homomorphism $G \rightarrow \operatorname{Aut}(R) / \operatorname{Inn}(R)$. Then one can define a gerbal action of $G$ on $\mathcal{C}$. Namely, we choose any lifting of this homomorphism to a map $s: G \rightarrow \operatorname{Aut}(R)$ (not necessarily a group homomorphism!). Let $m: R \otimes M \rightarrow M$ be a left $A$-module. Then define a new 
$A$-module $F_{g} m: R \otimes M \rightarrow M$ by the formula

$$
\left(F_{g} m\right)(a, x)=m\left(s(g)^{-1} a, x\right) \text { for } a \in A, x \in M .
$$

If $f:(\rho, M) \rightarrow\left(\rho^{\prime}, M^{\prime}\right)$ is a morphism between $A$-modules, then define $F_{g}(f)=f$ as linear map between underlying $\mathbb{C}$-vector spaces. Clearly, $F_{g}: \mathcal{C} \rightarrow \mathcal{C}$ is a functor. Moreover, it is easy to see that $F$ is a gerbal action of $G$ on $\mathcal{C}$ and we obtain the following:

Corollary 2.13. If $G$ acts on an associative $\mathbb{C}$-algebra by outer automorphisms, then there is a canonically defined class $a \in H^{3}\left(G, Z(A)^{\times}\right)$. This class vanishes if and only if $G$ acts on $A$ by (genuine) automorphisms.

2.3.7. 2-groups arising from gerbal actions. Recall that if a group acts a vector space projectively, we obtain a representation of a central extension of the group. Likewise, if a group $G$ acts on a category $\mathcal{C}$ gerbally, we obtain a genuine action of a certain 2-group on this category.

Assume that there is a gerbal action $F$ of $G$ on some category $\mathcal{C}$, which gives us a third cohomology class $[a] \in H^{3}\left(G, \mathcal{Z}(\mathcal{C})^{\times}\right)$. Let $A \subset \mathcal{Z}(\mathcal{C})^{\times}$be an abelian subgroup and suppose that the cohomology class $[a] \in H^{3}\left(G, \mathcal{Z}(\mathcal{C})^{\times}\right)$lies in the image of the map $H^{3}(G, A) \rightarrow H^{3}\left(G, \mathcal{Z}(\mathcal{C})^{\times}\right)$. Then we have, for any pair $g, g^{\prime} \in G$, an isomorphism $c\left(g, g^{\prime}\right): F_{g} F_{g^{\prime}} \cong F_{g g^{\prime}}$ such that $a\left(g, g^{\prime}, g^{\prime \prime}\right) \in A$ for any $g, g^{\prime}, g^{\prime \prime} \in G$, where $a\left(g, g^{\prime}, g^{\prime \prime}\right)$ is as in $§ 2.3 .5$. We claim that there is a 2-group $\mathcal{G}$, with $\pi_{0}(\mathcal{G})=G$ and $\pi_{1}(\mathcal{G})=A$, and a genuine action of $\mathcal{G}$ on $\mathcal{C}$, which lifts the gerbal action of $G$ on $\mathcal{C}$ (in the obvious sense). Namely, let $\mathcal{G}$ be the category whose objects are $g \in G$, and morphisms are $\operatorname{Hom}_{\mathcal{G}}\left(g, g^{\prime}\right)=\emptyset$, if $g \neq g^{\prime}$, and $\operatorname{End}_{\mathcal{G}}(g)=l_{F_{g}}(A)$, where

$$
l_{F_{g}}: \pi_{1}(\mathbb{A U T}(\mathcal{C}))=\mathcal{Z}(\mathcal{C})^{\times} \rightarrow \operatorname{End}_{\mathbb{A U T}(\mathcal{C})}\left(F_{g}\right)
$$

is an isomorphism. (Let us recall that in a 2-group, the isomorphism $l_{x}: \pi_{1}=\operatorname{End}(I) \rightarrow$ $\operatorname{End}(x)$ is induced from the isomorphism $I \otimes x \cong x$.) The monoidal structure of $\mathcal{G}$ is given by $g \otimes g^{\prime}=g g^{\prime}$, with the associativity constraint

$$
\left(a\left(g, g^{\prime}, g^{\prime \prime}\right):\left(g \otimes g^{\prime}\right) \otimes g^{\prime \prime} \cong g \otimes\left(g^{\prime} \otimes g^{\prime \prime}\right)\right) \in \operatorname{End}_{\mathcal{G}}\left(g g^{\prime} g^{\prime \prime}\right) .
$$

It is clear that this monoidal structure makes $\mathcal{G}$ a 2-group. The action of $\mathcal{G}$ on $\mathcal{C}$ is tautological. Namely, $g$ acts on $\mathcal{C}$ by $F_{g}$.

\section{2-Dimensional Story: Gerbal Representations}

Having developed the formalism of gerbal representations of groups, we now wish to apply it to a particular group, which we call $G L_{\infty, \infty}$. We will construct a gerbal representation of this group on a category of modules over a Clifford algebra. Presumably, it may also be realized using the corresponding "double infinite" Grassmannian (by analogy with the 1-dimensional story). We give some indications of how to do this in 33.4 , but this Grassmannian is a rather complicated geometric object that requires further study.

Alternatively, the gerbal action of $G L_{\infty, \infty}$ on this category may be viewed as a genuine representation of a "2-group", which is a $B \mathbb{C}^{\times}$-central extension of $G L_{\infty, \infty}$. We start this section by introducing these notions and develop the formalism necessary to describe the gerbal actions of the group $G L_{\infty, \infty}$. We then use this formalism to define gerbal representations of $G L_{\infty, \infty}$.

\subsection{The Lie algebra $\mathfrak{g l}_{\infty, \infty}$ and the group $G L_{\infty, \infty}$.}


3.1.1. Definition. We start by realizing $\mathfrak{g l}_{\infty}$ as a functor from associative rings to associative rings. Namely, for any ring $R$, regard $R((t))$ as a topological right $R$-module with the $t$-adic topology. Then define $\mathfrak{g l}_{\infty}(R)$ as the ring of continuous endomorphisms of $R((t))$ viewed as a right $R$-module (so that $\mathfrak{g l}_{\infty}(R)$ acts on $R((t))$ from the left). Now we define

$$
\mathfrak{g l}_{\infty, \infty}:=\mathfrak{g l}_{\infty}\left(\mathfrak{g l}_{\infty}\right)
$$

We also set

$$
G L_{\infty, \infty}:=\left\{g \in \mathfrak{g l}_{\infty, \infty}, g \text { is invertible }\right\} .
$$

Let us described the Lie algebra in more concrete terms. If we give $K=\mathbb{C}((t))$ the topological basis $\left\{t^{i}\right\}$, then elements in $\mathfrak{g l}_{\infty}$ could be regarded as $\infty \times \infty$-matrices $A=\left(A_{i j}\right)_{i, j \in \mathbb{Z}}$ which act on $\mathbb{C}((t))$ by the formula

$$
A\left(t^{j}\right)=\sum_{i \in \mathbb{Z}} A_{i j} t^{i}
$$

It is easy to see that

$$
\mathfrak{g l}_{\infty}=\left\{\begin{array}{c}
\left(A_{i j}\right)_{i, j \in \mathbb{Z}}, A_{i j} \in \mathbb{C} \mid \forall m \in \mathbb{Z}, \exists n \in \mathbb{Z}, \\
\text { such that whenever } i<m, j>n, A_{i j}=0
\end{array}\right\} .
$$

Therefore,

$$
\mathfrak{g l}_{\infty, \infty}=\left\{\begin{array}{c}
\left(A_{i j}\right)_{i, j \in \mathbb{Z}}, A_{i j} \in \mathfrak{g l}_{\infty} \mid \forall m \in \mathbb{Z}, \exists n \in \mathbb{Z}, \\
\text { such that whenever } i<m, j>n, A_{i j}=0
\end{array}\right\} .
$$

From this presentation, it is clear that $\mathfrak{g l}_{\infty, \infty}$ acts on

$$
\mathbb{K}:=\mathbb{C}((t))((s))
$$

by the following formula. If we represent an element in $\mathfrak{g l}_{\infty, \infty}$ by $A=\left(A_{i j}\right)_{i, j \in \mathbb{Z}}$ and $A_{i j}=\left(A_{i j, m n}\right)_{m, n \in \mathbb{Z}}$. Then

$$
A\left(t^{n} s^{j}\right)=\sum_{m, n \in \mathbb{Z}} A_{i j, m n} t^{m} s^{i}
$$

Observe that $G L_{\infty, \infty}$ acts on $\mathbb{K}$ continuously by the same formula as above.

The topology on $\mathbb{K}$ is given as follows: a basis of open neighborhoods of $0 \in \mathbb{K}$ consists of the subspaces

$$
s^{m} \mathbb{C}((t))[[s]]+\sum_{i \in \mathbb{Z}} t^{m_{i}} s^{i} \mathbb{C}[[t]]
$$

for some $m, m_{i} \in \mathbb{Z}$. The following lemma is proved in [Osi2], Proposition 3 .

Lemma 3.1. The action of $\mathfrak{g l}_{\infty, \infty}$ on $\mathbb{K}$ is continuous with respect to this topology.

Remark 3.2. In Osi2, Proposition 3, the author also shows that the algebra of continuous endomorphisms of $\mathbb{K}$ is larger that $\mathfrak{g l}_{\infty, \infty}$. 
3.1.2. Lattices in $\mathbb{K}=\mathbb{C}((t))((s))$. Let us recall the following construction of completed tensor products of topological vector spaces from [BD], §3.6.1. All topological vector spaces in this subsection are assumed to be linearly topologized, complete and separated with respect to the topology.

Let $V_{i}, i=1,2, \ldots, n$, be topological vector spaces. Then one defines the completed tensor product $V_{1} \vec{\otimes} V_{2} \vec{\otimes} \cdots \vec{\otimes} V_{n}$ as the completion of the plain tensor product $V_{1} \otimes V_{2} \otimes$ $\cdots \otimes V_{n}$ with respect to a topology in which a vector subspace $U \subset V_{1} \otimes V_{2} \otimes \cdots \otimes V_{n}$ is open if and only if for any $1 \leq i \leq n$ and $v_{i+1} \in V_{i+1}, \ldots, v_{n} \in V_{n}$, there exists an open $P \subset V_{i}$ such that $U \supset V_{1} \otimes \cdots V_{i-1} \otimes P \otimes v_{i+1} \otimes \cdots v_{n}$. Observe that in general, $V_{1} \vec{\otimes} V_{2} \nsucceq V_{2} \vec{\otimes} V_{1}$.

If $U$ be a topological vector space and $V \cong \mathbb{C}^{n}$ is a finite-dimensional vector space, then $U \vec{\otimes} V \cong U^{n}$ as a topological vector space. Therefore, if $U$ is a Tate vector space, so is $U \vec{\otimes} V$. Furthermore, if $V_{1} \rightarrow V_{2}$ is a closed embedding and $V_{2} / V_{1}$ is equipped with the quotient topology, then $U \vec{\otimes} V_{1} \rightarrow U \vec{\otimes} V_{2}$ is a closed embedding and $\left(U \vec{\otimes} V_{2}\right) /\left(U \vec{\otimes} V_{1}\right)$ with the quotient topology is canonically isomorphic to $U \vec{\otimes}\left(V_{2} / V_{1}\right)$.

It is easy to check if $V_{1}=\mathbb{C}((t))$ and $V_{2}=\mathbb{C}((s))$, with the usual adic topologies, then $V_{1} \vec{\otimes} V_{2}$ is isomorphic to $\mathbb{K}$ as a topological vector space. In this paper one could replace $\mathbb{K}$ by any other topological vector space of the form $U \vec{\otimes} V$, where $U$ and $V$ are two non-compact Tate vector spaces (see \$1.1.1).

Definition 3.3. Let $U$ and $V$ be two Tate vector spaces. We will call $\mathbb{L} \subset U \vec{\otimes} V$ a lattice if (i) $\mathbb{L}$ is closed in $U \vec{\otimes} V$; and (ii) there exist linearly compact open subspaces $P_{1} \subset P_{2}$ of $V$, such that $U \vec{\otimes} P_{1} \subset \mathbb{L} \subset U \vec{\otimes} P_{2}$.

It is clear that the intersection of two lattices is again a lattice.

For any pair of linearly compact open subspaces $P_{1} \subset P_{2} \subset V,\left(U \vec{\otimes} P_{2}\right) /\left(U \vec{\otimes} P_{1}\right)$ is a Tate vector space. Since $\mathbb{L}$ is closed, if $U \vec{\otimes} P_{1} \subset \mathbb{L} \subset U \vec{\otimes} P_{2}$, the exact sequence

$$
0 \rightarrow \mathbb{L} /\left(U \vec{\otimes} P_{1}\right) \rightarrow\left(U \vec{\otimes} P_{2}\right) /\left(U \vec{\otimes} P_{1}\right) \rightarrow\left(U \vec{\otimes} P_{2}\right) / \mathbb{L} \rightarrow 0
$$

is an exact sequence in the category of Tate vector spaces (see \$1.1.1).

Unlike the case of $K$, two lattices $\mathbb{L}, \mathbb{L}^{\prime}$ in $U \vec{\otimes} V$ are not necessarily commensurable. But we have the following weaker statement:

Lemma 3.4. For any two lattices $\mathbb{L}^{\prime}, \mathbb{L}$ in $U \vec{\otimes} V$ (where $U, V$ are Tate vector spaces, as above) the quotients $\mathbb{L} /\left(\mathbb{L} \cap \mathbb{L}^{\prime}\right)$ and $\mathbb{L}^{\prime} /\left(\mathbb{L} \cap \mathbb{L}^{\prime}\right)$, endowed with the quotient topology, are Tate vector spaces.

Proof. It is sufficient to consider the case when $\mathbb{L} \subset \mathbb{L}^{\prime}$. Let $P$ be a linearly compact open subspace of $V$ such that $U \vec{\otimes} P \supset \mathbb{L}^{\prime} \supset \mathbb{L}$. Then $\mathbb{L}^{\prime} / \mathbb{L}$ is a closed subspace of $U \vec{\otimes} P / \mathbb{L}$. Since $U \vec{\otimes} P / \mathbb{L}$ is a Tate vector space, so is $\mathbb{L}^{\prime} / \mathbb{L}$.

In the case when $U \vec{\otimes} V=\mathbb{K}$, we have the following lattice in $\mathbb{K}$ :

$$
\mathcal{O}_{\mathbb{K}}:=\mathbb{C}((t))[[s]] \cong K \vec{\otimes} \mathcal{O}_{K} .
$$

Note that condition (ii) of Definition 3.3 may be written as

$$
s^{N} \mathcal{O}_{\mathbb{K}} \subset \mathbb{L} \subset s^{-N} \mathcal{O}_{\mathbb{K}}
$$

for sufficiently large integer $N$. Here is a more general example of a lattice in $\mathbb{K}$ :

$$
\mathbb{L}=s^{N} \mathbb{C}((t))[[s]] \oplus \bigoplus_{i=-M}^{N-1} s^{i} t^{m_{i}} \mathbb{C}[[t]], \quad m_{i} \in \mathbb{Z}, M \in \mathbb{Z}_{+} .
$$


The following lemma shows that all other lattices in $\mathbb{K}$ may be obtained from $\mathcal{O}_{\mathbb{K}}$ by the $G L_{\infty, \infty}$-action.

Lemma 3.5. (i) For any $g \in G L_{\infty, \infty}, g \mathcal{O}_{\mathbb{K}}$ is a lattice in $\mathbb{K}$.

(ii) $G L_{\infty, \infty}$ acts transitively on the set of lattices in $\mathbb{K}$.

Proof. (i) It is clear that for any $g \in G L_{\infty, \infty}$ there exists an integer $N$ such that $s^{N} \mathcal{O}_{\mathbb{K}} \subset g \mathcal{O}_{\mathbb{K}} \subset s^{-N} \mathcal{O}_{\mathbb{K}}$. Since $\mathcal{O}_{\mathbb{K}}$ is closed in $\mathbb{K}$ and the action of $g$ is continuous, $g \mathcal{O}_{\mathbb{K}}$ is also closed in $\mathbb{K}$.

(ii) Let $\mathbb{L}$ be a lattice such that $s^{N} \mathcal{O}_{\mathbb{K}} \subset \mathbb{L} \subset s^{-N} \mathcal{O}_{\mathbb{K}}$. Then we have the exact sequence

$$
0 \rightarrow \mathbb{L} / s^{N} \mathcal{O}_{\mathbb{K}} \rightarrow s^{-N} \mathcal{O}_{\mathbb{K}} / s^{N} \mathcal{O}_{\mathbb{K}} \rightarrow s^{-N} \mathcal{O}_{\mathbb{K}} / \mathbb{L} \rightarrow 0
$$

By Remark 1.2, we could assume that $s^{-N} \mathcal{O}_{\mathbb{K}} / s^{N} \mathcal{O}_{\mathbb{K}}=\mathbb{L} / s^{N} \mathcal{O}_{\mathbb{K}} \oplus L^{\prime}$ for some $L^{\prime} \cong$ $s^{-N} \mathcal{O}_{\mathbb{K}} / \mathbb{L}$. For any $k \in \mathbb{Z}$, we choose an isomorphism

$$
s^{-N} \mathcal{O}_{\mathbb{K}} / s^{N} \mathcal{O}_{\mathbb{K}} \cong \sum_{i=0}^{2 N-1} s^{(2 k-1) N+i} \mathbb{C}((t))
$$

and let the image of $\mathbb{L} / s^{N} \mathcal{O}_{\mathbb{K}}$ and $L^{\prime}$ in $\sum_{i=0}^{2 N-1} s^{(2 k-1) N+i} \mathbb{C}((t))$ be $L_{k}$ and $L_{k}^{\prime}$ respectively. We can always make $L_{0}=\mathbb{L} \cap \sum_{i=0}^{2 N-1} s^{-N+i} \mathbb{C}((t))$. Let us define an automorphism $g: \mathbb{K} \rightarrow \mathbb{K}$ as follows. We write

$$
\mathbb{K}=\sum_{k<0} \sum_{i=0}^{2 N-1} s^{2 k N+i} \mathbb{C}((t)) \oplus \prod_{k \geq 0} \sum_{i=0}^{N-1} s^{2 k N+i} \mathbb{C}((t))
$$

For each $k$, we define an isomorphism

$$
g_{k}: \sum_{i=0}^{2 N-1} s^{2 k N+i} \mathbb{C}((t)) \cong L_{k}+L_{k+1}^{\prime}
$$

and then set

$$
g=\sum_{k<0} g_{k}+\prod_{k \geq 0} g_{k}
$$

It is clear that $g \in G L_{\infty, \infty}$ and $g \mathcal{O}_{\mathbb{K}}=\mathbb{L}$.

3.2. A gerbal representation of $G L_{\infty, \infty}$ on a category of Clifford modules. We wish to define a gerbal representation of $G L_{\infty, \infty}$ on a certain abelian category realizing a non-trivial third cohomology class. Recall that according to Definition 2.8, a gerbal representation of a group $G$ on an abelian category $\mathcal{C}$ is a homomorphism of groups $F: G \rightarrow \pi_{0}(\mathbb{G L}(\mathcal{C}))$, where $\mathbb{G} \mathbb{L}(\mathcal{C})$ is the category of $\mathbb{C}$-linear auto-equivalences of $\mathcal{C}$ (see 2.3 .2 ). More explicitly, this means assigning every $g \in G$ an auto-equivalence $F_{g}: \mathcal{C} \rightarrow \mathcal{C}$ such that $F_{e} \cong 1_{\mathcal{C}}$ and $F_{g} F_{g^{\prime}} \cong F_{g g^{\prime}}$ (see 2.3 .4 ).

Theorem 2.10 shows that a gerbal representation of $G$ on $\mathcal{C}$ gives rise to a third cohomology class of $G$ with coefficients in $\mathcal{Z}(\mathcal{C})^{\times}$, the (abelian) group of invertible elements of the center $\mathcal{Z}(\mathcal{C})$ of the category $\mathcal{C}$. Furthermore, as explained in 22.3 .7 , we obtain a 2-group $\mathcal{G}$ equipped with a genuine action on $\mathcal{C}$.

This is analogous to the notion of projective representation of a group $G$ on a vector space $V$. Such a representation gives rise to a second cohomology class of $G$ and to a canonical central extension of $G$ which genuinely acts on $V$. In the 1-dimensional story 
we naturally obtain a projective representation of $G L_{\infty}$ on a module over a Clifford algebra, as explained in $\$ 1$. Therefore it is natural to guess that one can construct a gerbal action of $G L_{\infty, \infty}$ on a category of modules over a Clifford algebra. What could this category be? A naive guess is that it should be a category of modules over the Clifford algebra $\mathrm{Cl}\left(\mathbb{K} \oplus \mathbb{K}^{*}\right)$. However, this cannot be true since $G L_{\infty, \infty}$ acts by automorphisms on $\mathrm{Cl}\left(\mathbb{K} \oplus \mathbb{K}^{*}\right)$ and therefore, it acts on the category of modules genuinely (that is, the corresponding third cohomology class is equal to 0 ). It turns out that the correct Clifford algebra is the Clifford algebra $\mathrm{Cl}\left(\mathcal{O}_{\mathbb{K}} \oplus \mathcal{O}_{\mathbb{K}}^{*}\right)$ associated to the lattice $\mathcal{O}_{\mathbb{K}}$ of $\mathbb{K}$. In 55.3 we will show that the corresponding third cohomology class is the non-zero class $\left[E_{3}\right] \in H^{3}\left(G L_{\infty, \infty}, \mathbb{C}^{\times}\right)$constructed in 44.2 .

3.2.1. More on lattices in $\mathbb{K}=\mathbb{C}((t))((s))$. Let $U$ and $V$ be Tate vector spaces. Observe that lattices of $U \vec{\otimes} V$ in general are not open subspaces of $U \vec{\otimes} V$. Let $\mathbb{L}$ be a lattice. We can endow $(U \vec{\otimes} V) / \mathbb{L}$ with the quotient topology. Then $(U \vec{\otimes} V) / \mathbb{L}$ is an ind-Tate vector space, i.e. $(U \vec{\otimes} V) / \mathbb{L}=\lim _{\overrightarrow{i \in I}} V_{i}$, where $V_{i}$ are Tate vector spaces, $V_{i} \rightarrow V_{j}$ are closed embeddings, and a subspace $W \subset(U \vec{\otimes} V) / \mathbb{L}$ is open if and only if $W \cap V_{i}$ is open in $V_{i}$ for any $i \in I$. For example, if $\mathbb{L}=\mathcal{O}_{\mathbb{K}} \subset \mathbb{K}$, then

$$
\mathbb{K} / \mathcal{O}_{\mathbb{K}} \cong s^{-1} \mathbb{C}((t))\left[s^{-1}\right] \cong \mathbb{C}((t))^{\mathbb{N}}
$$

A basis of open neighborhood of $0 \in \mathbb{K} / \mathcal{O}_{\mathbb{K}}$ could be given by the sets $\sum_{i \leq-1} s^{i} t^{m_{i}} \mathbb{C}[[t]]$ for different collections $\left(m_{i}\right)$.

Let $\mathbb{K}^{*}:=K^{*} \vec{\otimes} K^{*} \cong \mathbb{C}((t))((s)) d t d s$. We have a natural non-degenerate symmetric bilinear form on $\mathbb{K} \oplus \mathbb{K}^{*}$ induced by the residue pairing. That is,

$$
(f, \omega)=\operatorname{Res}_{t=0} \operatorname{Res}_{s=0} f \omega \quad f \in \mathbb{K}, \omega \in \mathbb{K}^{*} .
$$

It is clear that if $\mathbb{L} \subset \mathbb{K}$ is a lattice, then $\mathbb{L}^{\perp} \subset \mathbb{K}^{*}$ is also a lattice. We have a non-degenerate symmetric bilinear form on $\mathbb{L} \oplus \mathbb{K}^{*} / \mathbb{L}^{\perp}$.

Definition 3.6. Let $\mathbb{L}$ be a lattice in $U \vec{\otimes} V$, where $U$ and $V$ are Tate vector spaces. We will call $L \subset \mathbb{L}$ a secondary lattice of $\mathbb{L}$ if $L$ is closed in $\mathbb{L}$ and for any linearly compact open subspace $P \subset V$ such that $U \vec{\otimes} P \subset \mathbb{L}, L /(L \cap U \vec{\otimes} P))$ is linearly compact open in $\mathbb{L} /(U \vec{\otimes} P)$.

If $\mathbb{L}=\mathbb{C}((t))[[s]]$, a lattice in $\mathbb{K}=\mathbb{C}((t))((s))$, then the following is an example of a secondary lattice:

$$
L=\prod_{n \geq 0} s^{m_{i}} \mathbb{C}[[t]], \quad m_{i} \in \mathbb{Z} .
$$

Let $L$ be a secondary lattice of $\mathbb{L}$. Denote by $L^{\perp} \subset(U \vec{\otimes} V)^{*} / \mathbb{L}^{\perp}$ its orthogonal complement, modulo $\mathbb{L}^{\perp}$. Observe that for any linearly compact open subspace $P \subset V$ such that $U \vec{\otimes} P \subset \mathbb{L},(U \vec{\otimes} P)^{\perp} / \mathbb{L}^{\perp}$ is a Tate vector subspace of $(U \vec{\otimes} V)^{*} / \mathbb{L}^{\perp}$. It is clear that $L^{\perp} \cap\left((U \vec{\otimes} P)^{\perp} / \mathbb{L}^{\perp}\right)$ is a linearly compact open subspace of $(U \vec{\otimes} P)^{\perp} / \mathbb{L}^{\perp}$. Therefore, $L^{\perp}$ is an open subspace of $(U \vec{\otimes} V)^{*} / \mathbb{L}^{\perp}$.

Lemma 3.7. Denote by $\left(L^{\perp}\right)^{\perp} \subset \mathbb{L}$ the orthogonal complement of $L^{\perp}$. Then $\left(L^{\perp}\right)^{\perp}=$ $L$.

Proof. Observe that $L$ is a secondary lattice in $\mathbb{L}$ if and only if for any linearly compact open subspace $P \subset V$ such that $U \vec{\otimes} P \subset \mathbb{L}, L /(L \cap(U \vec{\otimes} P))$ is linearly compact open 
in $\mathbb{L} /(U \vec{\otimes} P)$, and $L=\lim _{\overleftarrow{P}} L /(L \cap(U \vec{\otimes} P))$. Indeed, there is a natural injection $L \rightarrow$ $\lim _{\overleftarrow{P}} L /(L \cap(U \vec{\otimes} P))$, which is a surjection if and only if $L$ is closed.

Then $\left(L^{\perp}\right)^{\perp}=\lim _{\overleftarrow{P}}\left(L^{\perp} \cap(U \vec{\otimes} P)^{\perp} / \mathbb{L}^{\perp}\right)^{\perp}$, where $\left(L^{\perp} \cap(U \vec{\otimes} P)^{\perp} / \mathbb{L}^{\perp}\right)^{\perp}$ is the orthogonal complement of $L^{\perp} \cap(U \vec{\otimes} P)^{\perp} / \mathbb{L}^{\perp}$ in $\mathbb{L} /(U \vec{\otimes} P)$, under the natural pairing between $\mathbb{L} /(U \vec{\otimes} P)$ and $(U \vec{\otimes} P)^{\perp} / \mathbb{L}^{\perp}$. However, it is clear that $\left(L^{\perp} \cap(U \vec{\otimes} P)^{\perp} / \mathbb{L}^{\perp}\right)^{\perp}=$ $L /(L \cap U \vec{\otimes} P)$. The lemma follows.

Therefore $L \oplus L^{\perp}$ is a maximal isotropic subspace of $\mathbb{L} \oplus(U \vec{\otimes} V)^{*} / \mathbb{L}^{\perp}$. We will call such subspaces Lagrangian.

3.2.2. The category of Clifford modules. From now on we will consider lattices $\mathbb{L} \subset$ $\mathbb{K}=\mathbb{C}((t))((s))$. Let

$$
\mathrm{Cl}_{\mathbb{L}}:=\mathrm{Cl}\left(\mathbb{L} \oplus\left(\mathbb{K}^{*} / \mathbb{L}^{\perp}\right)\right)
$$

be the Clifford algebra associated to the space $\mathbb{L} \oplus\left(\mathbb{K}^{*} / \mathbb{L}^{\perp}\right)$ equipped with a nondegenerate symmetric bilinear form defined above. Let $L$ be a secondary lattice of $\mathbb{L}$. Since $L \oplus L^{\perp}$ is a Lagrangian subspace of $\mathbb{L} \oplus \mathbb{K}^{*} / \mathbb{L}^{\perp}$, the exterior algebra $\bigwedge\left(L \oplus L^{\perp}\right)$ is a subalgebra of $\mathrm{Cl}_{\mathbb{L}}$. We define a $\mathrm{Cl}_{\mathbb{L}}$-module

$$
M_{L}=\operatorname{Ind}_{\bigwedge\left(L \oplus L^{\perp}\right)}^{\mathrm{Cl} \mathbb{L}_{\mathbb{L}}}(\mathbb{C}|0\rangle)
$$

Observe that $M_{L}$ is not a discrete $\mathrm{Cl}_{\mathbb{L}}$-module. Nevertheless, we still have the following

Lemma 3.8. $M_{L}$ is an irreducible $\mathrm{Cl}_{\mathbb{L}}$-module.

Proof. We can always find a subspace $L^{\prime} \subset \mathbb{L}$ such that $\mathbb{L}=L \oplus L^{\prime}$ and that $\mathbb{K}^{*} / \mathbb{L}^{\perp}=$ $L^{\perp} \oplus L^{\prime \perp}$. Therefore, $M_{L} \cong \bigwedge\left(L^{\prime} \oplus L^{\prime \perp}\right)|0\rangle$, and any element in $M_{L}$ could be expressed as a finite sum $m=\sum_{i_{1}, \ldots, i_{r}} v_{i_{1}} \cdots v_{i_{r}}|0\rangle$ with $v_{i_{j}} \in L^{\prime} \oplus L^{\prime \perp}$. Let $W$ be the span of these $v_{i_{j}}$. This is a finite-dimensional subspace in $L^{\prime} \oplus L^{\prime \perp}$. One can always find a finite-dimensional subspace $W^{*} \subset L \oplus L^{\perp}$ such that the non-degenerate symmetric bilinear form on $\mathbb{L} \oplus \mathbb{K}^{*} / \mathbb{L}$ restricts to a non-degenerate symmetric bilinear form on $W \oplus W^{*}$. Let $\mathrm{Cl}_{W}=\mathrm{Cl}\left(W \oplus W^{*}\right)$ be the Clifford algebra associated to this bilinear form. This is a subalgebra of $\mathrm{Cl}_{\mathbb{L}}$. It is clear that $m \in \mathrm{Cl}_{W}|0\rangle=\operatorname{Ind}_{\Lambda W^{*}}^{\mathrm{Cl}_{W}}(\mathbb{C}|0\rangle)$. From the theory of Clifford algebras modeled on finite-dimensional vector spaces we know that there exists some $a \in \mathrm{Cl}_{W} \subset \mathrm{Cl}_{\mathbb{L}}$ such that $a(m)=|0\rangle$. This proves that $M_{L}$ is irreducible.

Lemma 3.9. $\operatorname{Hom}_{\mathrm{Cl}_{\mathrm{L}}}\left(M_{L}, M_{L^{\prime}}\right) \neq 0$ if and only if $L$ and $L^{\prime}$ are commensurable with each other. In that case, there exists a canonical isomorphism

$$
\operatorname{Hom}_{\mathrm{Cl}_{\mathbb{L}}}\left(M_{L}, M_{L^{\prime}}\right) \cong \operatorname{det}\left(L \mid L^{\prime}\right)
$$

such that the same diagram as in Lemma 1.11 holds.

Proof. Assume that $\phi \in \operatorname{Hom}_{\mathrm{Cl}_{\mathbb{L}}}\left(M_{L}, M_{L^{\prime}}\right)$ is non-zero. Then $\phi\left(|0\rangle_{L}\right)$ is an element in $M_{L^{\prime}}$ which is annihilated by $L \oplus L^{\perp}$. As in the proof of the previous lemma, one can find a finite-dimensional Clifford algebra $\mathrm{Cl}_{W} \subset \mathrm{Cl}_{\mathbb{L}}$ such that $\phi\left(|0\rangle_{L}\right) \in \mathrm{Cl}_{W}|0\rangle_{L^{\prime}}=$ $\operatorname{Ind}_{\wedge W^{*}}^{\mathrm{Cl}_{W}}\left(\mathbb{C}|0\rangle_{L^{\prime}}\right)$. Then it is clear that $\phi\left(|0\rangle_{L}\right)$ is annihilated by $W^{\perp} \cap\left(L^{\prime} \oplus L^{\prime \perp}\right)$. Therefore, $W^{\perp} \cap\left(L^{\prime} \oplus L^{\prime \perp}\right) \subset L \oplus L^{\perp}$. Since $\left(L^{\prime} \oplus L^{\prime \perp}\right) /\left(W^{\perp} \cap\left(L^{\prime} \oplus L^{\prime \perp}\right)\right) \cong W^{*}$ is finite-dimensional, we obtain that $L^{\prime} /\left(L^{\prime} \cap L\right)$ is also finite-dimensional. Since $M_{L}$ 
and $M_{L^{\prime}}$ are irreducible, $\phi$ is an isomorphism. Applying the same argument to $\phi^{-1}$, we obtain that $L /\left(L \cap L^{\prime}\right)$ is finite-dimensional.

Before we prove the second statement of the lemma, we claim that the $\bigwedge\left(L \oplus L^{\perp}\right)$ invariant subspace of $M_{L}$ is the line $\mathbb{C}|0\rangle$. Indeed, assume that $m \in M_{L}$ is invariant under $\bigwedge\left(L \oplus L^{\perp}\right)$. Then, as in the proof of the previous lemma, we can assume that $m \in \mathrm{Cl}_{W}|0\rangle=\operatorname{Ind}_{\Lambda W^{*}}^{\mathrm{Cl}_{W}}(\mathbb{C}|0\rangle)$. Then $m$ is invariant under $\wedge W^{*}$. By the theory of finite dimensional Clifford algebras, $m \in \mathbb{C}|0\rangle$.

Now assume that $L$ and $L^{\prime}$ are commensurable. As in the proof of Lemma 1.11, Hom $_{\mathrm{Cl}_{\mathbb{L}}}\left(M_{L}, M_{L^{\prime}}\right)$ is canonically isomorphic to the $\bigwedge\left(L \oplus L^{\perp}\right)$-invariant subspace in $M_{L^{\prime}}$, which is either zero- or one-dimensional. Indeed, if this subspace is not zero, then $M_{L^{\prime}} \cong M_{L}$ and we have just seen that the $\bigwedge\left(L \oplus L^{\perp}\right)$-invariant subspace in $M_{L}$ is one-dimensional.

Let $V=\left(L \oplus L^{\perp}\right) \cap\left(L^{\prime} \oplus L^{\perp}\right)$. Then $V^{\perp} / V$ is finite-dimensional and it carries a non-degenerate symmetric bilinear form. Denote $\mathrm{Cl}\left(V^{\perp} / V\right)$ to be the corresponding Clifford algebra. The $V$-invariant subspace $M_{L^{\prime}}^{V}$ in $M_{L^{\prime}}$ is naturally a module over $\mathrm{Cl}\left(V^{\perp} / V\right)$. Let $\mathrm{Cl}\left(V^{\perp} / V\right)|0\rangle_{L^{\prime}}$ be the submodule containing the vacuum vector $|0\rangle_{L^{\prime}}$. We know that $\left(L \oplus L^{\perp}\right) / V$ is a Lagrangian subspace of $V^{\perp} / V$. The theory of finitedimensional Clifford algebras implies that the $\Lambda\left(\left(L \oplus L^{\perp}\right) / V\right)$-invariant subspace in $\mathrm{Cl}\left(V^{\perp} / V\right)|0\rangle_{L^{\prime}}$ is canonically isomorphic to $\bigwedge^{\text {top }} L /\left(L \cap L^{\prime}\right) \otimes\left(\bigwedge^{\text {top }} L^{\prime} /\left(L \cap L^{\prime}\right)\right)^{-1}$. That is, the $\bigwedge\left(L \oplus L^{\perp}\right)$-invariant subspace in $M_{L^{\prime}}$ is canonically isomorphic to $\operatorname{det}\left(L \mid L^{\prime}\right)$.

Definition 3.10. $\mathcal{C}_{\mathbb{L}}^{\mathrm{ss}}$ is the semi-simple abelian category, whose objects are $\mathrm{Cl}_{\mathbb{L}^{-}}$ modules that are direct sums of $M_{L}$, with $L$ being secondary lattices of $\mathbb{L}$, and morphisms are homomorphisms of these $\mathrm{Cl}_{\mathbb{L}}$-modules.

Let $g \in G L_{\infty, \infty}$. Let $\mathbb{L} \subset \mathbb{K}$ be a lattice, and $L \subset \mathbb{L}$ a secondary lattice. It is clear that $g L \subset g \mathbb{L}$ is a secondary lattice. We define, for any $g \in G L_{\infty, \infty}$, a functor

$$
T_{g}: \mathcal{C}_{\mathbb{L}}^{\mathrm{ss}} \rightarrow \mathcal{C}_{g \mathbb{L}}^{\mathrm{ss}}
$$

which sends $M_{L}$ to $M_{g L}$, and the map of Hom's is defined as follows: for $L, L^{\prime} \subset \mathbb{L}$ commensurable, we have

$$
\operatorname{Hom}_{\mathrm{Cl}_{\mathbb{L}}}\left(M_{L}, M_{L^{\prime}}\right) \cong \operatorname{det}\left(L \mid L^{\prime}\right) \cong \operatorname{det}\left(g L \mid g L^{\prime}\right) \cong \operatorname{Hom}_{\mathrm{Cl}_{g \mathbb{L}}}\left(M_{g L}, M_{g L^{\prime}}\right) .
$$

This isomorphism gives us a map $\operatorname{Hom}_{\mathrm{Cl}_{\mathbb{L}}}\left(M_{L}, M_{L^{\prime}}\right) \rightarrow \operatorname{Hom}_{\mathrm{Cl}_{g \mathbb{L}}}\left(M_{g L}, M_{g L^{\prime}}\right)$.

3.2.3. Gerbal representation of $G L_{\infty, \infty}$. The first main theorem of this paper is the following:

Theorem 3.11. There is a natural gerbal representation of $G L_{\infty, \infty}$ on the category $\mathcal{C}_{\mathbb{L}}^{\mathrm{ss}}$. These representations are equivalent to each other for all lattices $\mathbb{L} \subset \mathbb{K}$.

Proof. We will construct for any lattices two $\mathbb{L}, \mathbb{L}^{\prime} \subset \mathbb{K}$, an equivalence of categories

$$
\Xi_{\mathbb{L}, \mathbb{L}^{\prime}}: \mathcal{C}_{\mathbb{L}}^{\mathrm{ss}} \rightarrow \mathcal{C}_{\mathbb{L}^{\prime}}^{\mathrm{ss}}
$$

such that:

(i) $\Xi_{\mathbb{L}^{\prime}, \mathbb{L}^{\prime \prime}} \circ \Xi_{\mathbb{L}, \mathbb{L}^{\prime}} \cong \Xi_{\mathbb{L}, \mathbb{L}^{\prime \prime}}$;

(ii) For any $g \in G L_{\infty, \infty}, \Xi_{g \mathbb{L}, g \mathbb{L}^{\prime}} \circ T_{g} \cong T_{g} \circ \Xi_{\mathbb{L}, \mathbb{L}^{\prime}}$.

It is enough to construct such functors for pairs $\mathbb{L} \supset \mathbb{L}^{\prime}$, so that (i) and (ii) hold. Then we extend the definition to any pair $\mathbb{L}, \mathbb{L}^{\prime}$ by $\Xi_{\mathbb{L}, \mathbb{L}^{\prime}}=\Xi_{\mathbb{L}^{\prime}, \mathbb{L} \cap \mathbb{L}^{\prime}}^{-1} \circ \Xi_{\mathbb{L}, \mathbb{L} \cap \mathbb{L}^{\prime}}$, where $\Xi_{\mathbb{L}^{\prime}, \mathbb{L} \cap \mathbb{L}^{\prime}}^{-1}$ is any quasi-inverse functor of $\Xi_{\mathbb{L}^{\prime}, \mathbb{L} \cap \mathbb{L}^{\prime}}$. 
Therefore, we assume that $\mathbb{L} \supset \mathbb{L}^{\prime}$. According to Lemma $3.4, \mathbb{L} / \mathbb{L}^{\prime}$ is a Tate vector space. We therefore have the gerbe $\mathcal{D}_{\mathbb{L} / \mathbb{L}^{\prime}}$ of determinantal theories on $\mathbb{L} / \mathbb{L}^{\prime}$ (see Definition 1.7). Choose an object $\Delta_{\mathbb{L}, \mathbb{L}^{\prime}} \in \mathcal{D}_{\mathbb{L} / \mathbb{L}^{\prime}}$, that is, a determinantal theory. If $L \subset \mathbb{L}$ is a secondary lattice, then $L \cap \mathbb{L}^{\prime}$ is a secondary lattice of $\mathbb{L}^{\prime}$ and $L /\left(L \cap \mathbb{L}^{\prime}\right)$ is a linearly compact open subspace of $\mathbb{L} / \mathbb{L}^{\prime}$. Now $\Xi_{\mathbb{L}, \mathbb{L}^{\prime}}$ is defined on objects by the formula

$$
\Xi_{\mathbb{L}, \mathbb{L}^{\prime}}\left(M_{L}\right)=M_{L \cap \mathbb{L}^{\prime}} \otimes \Delta_{\mathbb{L}, \mathbb{L}^{\prime}}\left(L /\left(L \cap \mathbb{L}^{\prime}\right)\right)^{-1} .
$$

To define $\Xi_{\mathbb{L}, \mathbb{L}^{\prime}}$ on Hom's, observe that if $L$ and $L^{\prime}$ are two commensurable secondary lattices of $\mathbb{L}$, then we have a canonical isomorphism

$$
\operatorname{det}\left(L \mid L^{\prime}\right) \cong \operatorname{det}\left(L \cap \mathbb{L}^{\prime} \mid L^{\prime} \cap \mathbb{L}^{\prime}\right) \otimes \operatorname{det}\left(\frac{L}{L \cap \mathbb{L}^{\prime}} \mid \frac{L^{\prime}}{L^{\prime} \cap \mathbb{L}^{\prime}}\right)
$$

Since

$$
\operatorname{det}\left(\frac{L}{L \cap \mathbb{L}^{\prime}} \mid \frac{L^{\prime}}{L^{\prime} \cap \mathbb{L}^{\prime}}\right) \cong \operatorname{Hom}\left(\Delta_{\mathbb{L}, \mathbb{L}^{\prime}}\left(\frac{L^{\prime}}{L^{\prime} \cap \mathbb{L}^{\prime}}\right), \Delta_{\mathbb{L}, \mathbb{L}^{\prime}}\left(\frac{L}{L \cap \mathbb{L}^{\prime}}\right)\right)
$$

we have a canonical isomorphism

$$
\begin{aligned}
& \operatorname{Hom}_{\mathrm{Cl}_{\mathbb{L}}}(\left.M_{L}, M_{L^{\prime}}\right) \cong \\
& \quad \operatorname{Hom}_{\mathrm{Cl}_{\mathbb{L}^{\prime}}}\left(M_{L \cap \mathbb{L}^{\prime}} \otimes \Delta_{\mathbb{L}, \mathbb{L}^{\prime}}\left(L /\left(L \cap \mathbb{L}^{\prime}\right)\right)^{-1}, M_{L^{\prime} \cap \mathbb{L}^{\prime}} \otimes \Delta_{\mathbb{L}, \mathbb{L}^{\prime}}\left(L^{\prime} /\left(L^{\prime} \cap \mathbb{L}^{\prime}\right)\right)^{-1}\right) .
\end{aligned}
$$

This gives us the sough-after map

$$
\operatorname{Hom}_{\mathrm{Cl}_{\mathbb{L}}}\left(M_{L}, M_{L^{\prime}}\right) \rightarrow \operatorname{Hom}_{\mathrm{Cl}_{\mathbb{L}^{\prime}}}\left(\Xi_{\mathbb{L}, \mathbb{L}^{\prime}}\left(M_{L}\right), \Xi_{\mathbb{L}, \mathbb{L}^{\prime}}\left(M_{L^{\prime}}\right)\right) .
$$

(This explains the necessity of the second factor in formula (3.1).)

Now assume that $\mathbb{L} \supset \mathbb{L}^{\prime} \supset \mathbb{L}^{\prime \prime}$. Then

$$
\begin{aligned}
\Xi_{\mathbb{L}^{\prime}, \mathbb{L}^{\prime \prime}}\left(\Xi_{\mathbb{L}, \mathbb{L}^{\prime}}\left(M_{L}\right)\right) & =M_{L \cap \mathbb{L}^{\prime \prime}} \otimes \Delta_{\mathbb{L}, \mathbb{L}^{\prime}}\left(L /\left(L \cap \mathbb{L}^{\prime}\right)\right)^{-1} \otimes \Delta_{\mathbb{L}^{\prime}, \mathbb{L}^{\prime \prime}}\left(L \cap \mathbb{L}^{\prime} /\left(L \cap \mathbb{L}^{\prime \prime}\right)\right)^{-1}, \\
& =M_{L \cap \mathbb{L}^{\prime \prime}} \otimes\left(\Delta_{\mathbb{L}, \mathbb{L}^{\prime}} \otimes \Delta_{\mathbb{L}^{\prime}, \mathbb{L}^{\prime \prime}}\right)\left(L /\left(L \cap \mathbb{L}^{\prime \prime}\right)\right)^{-1}
\end{aligned},
$$

where we apply the canonical equivalence $\mathcal{D}_{\mathbb{L} / \mathbb{L}^{\prime}} \otimes \mathcal{D}_{\mathbb{L}^{\prime} / \mathbb{L}^{\prime \prime}} \cong \mathcal{D}_{\mathbb{L} / \mathbb{L}^{\prime \prime}}$ of Lemma 1.8

On the other hand

$$
\Xi_{\mathbb{L}, \mathbb{L}^{\prime \prime}}\left(M_{L}\right)=M_{L \cap \mathbb{L}^{\prime \prime}} \otimes \Delta_{\mathbb{L}, \mathbb{L}^{\prime \prime}}\left(L /\left(L \cap \mathbb{L}^{\prime \prime}\right)\right)^{-1} .
$$

Therefore, any choice of an isomorphism $\Delta_{\mathbb{L}, \mathbb{L}^{\prime}} \otimes \Delta_{\mathbb{L}^{\prime}, \mathbb{L}^{\prime \prime}} \cong \Delta_{\mathbb{L}, \mathbb{L}^{\prime \prime}}$ will give us an isomorphism of functors $\Xi_{\mathbb{L}^{\prime}, \mathbb{L}^{\prime \prime}} \circ \Xi_{\mathbb{L}, \mathbb{L}^{\prime}} \cong \Xi_{\mathbb{L}, \mathbb{L}^{\prime \prime}}$.

Next, let $g \in G L_{\infty, \infty}$. We have

$\left.T_{g}\left(\Xi_{\mathbb{L}, \mathbb{L}^{\prime}}\left(M_{L}\right)\right)=M_{g\left(L \cap \mathbb{L}^{\prime}\right)} \otimes \Delta_{\mathbb{L}, \mathbb{L}^{\prime}}\left(L /\left(L \cap \mathbb{L}^{\prime}\right)\right)^{-1}=M_{g\left(L \cap \mathbb{L}^{\prime}\right)} \otimes\left(g \Delta_{\mathbb{L}, \mathbb{L}^{\prime}}\right)\left(g L / g L \cap g \mathbb{L}^{\prime}\right)\right)^{-1}$, where $g\left(L \cap \mathbb{L}^{\prime}\right)$ is, by definition, the determinantal theory on $g \mathbb{L} / g \mathbb{L}^{\prime}$ which assigns to $\widetilde{L} \subset g \mathbb{L} / g \mathbb{L}^{\prime}$ the vector space $\Delta_{\mathbb{L} / \mathbb{L}^{\prime}}\left(g^{-1} \widetilde{L}\right)$. On the other hand, we have

$$
\Xi_{g \mathbb{L}, g \mathbb{L}^{\prime}}\left(T_{g}\left(M_{L}\right)\right)=M_{g L \cap g \mathbb{L}^{\prime}} \otimes \Delta_{g \mathbb{L}, g \mathbb{L}^{\prime}}\left(g L /\left(g L \cap g \mathbb{L}^{\prime}\right)\right)^{-1} .
$$

Note that $g \Delta_{\mathbb{L}, \mathbb{L}^{\prime}}$ and $\Delta_{g \mathbb{L}, g \mathbb{L}^{\prime}}$ are objects of the same groupoid $\mathcal{D}_{g \mathbb{L} / g \mathbb{L}^{\prime}}$. Therefore, the set of isomorphisms $g \Delta_{\mathbb{L}, \mathbb{L}^{\prime}} \cong \Delta_{g \mathbb{L}, g \mathbb{L}^{\prime}}$ is a $\mathbb{C}^{\times}$-torsor (in particular, non-empty). A choice of such an isomorphism will give rise to an isomorphism $T_{g} \circ \Xi_{\mathbb{L}, \mathbb{L}^{\prime}} \cong \Xi_{g \mathbb{L}, g \mathbb{L}^{\prime}} \circ T_{g}$.

Now we are ready to define a gerbal representation of $G L_{\infty, \infty}$ on $\mathcal{C}_{\mathbb{L}}^{\text {ss }}$. For $g \in G L_{\infty, \infty}$, we define the functor $F_{g}: \mathcal{C}_{\mathbb{L}}^{\text {ss }} \rightarrow \mathcal{C}_{\mathbb{L}}^{\text {ss }}$ as the composition

$$
\mathcal{C}_{\mathbb{L}}^{\mathrm{ss}} \stackrel{T_{g}}{\rightarrow} \mathcal{C}_{g \mathbb{L}}^{\mathrm{ss}} \stackrel{\Xi_{g \mathbb{L}, \mathbb{L}}}{\rightarrow} \mathcal{C}_{\mathbb{L}}^{\mathrm{ss}}
$$

Properties (i) and (ii) of $\Xi_{\mathbb{L}, \mathbb{L}^{\prime}}$ imply that $F_{g} \circ F_{g^{\prime}} \cong F_{g g^{\prime}}$. Thus, we obtain a gerbal representation of $G L_{\infty, \infty}$ on $\mathcal{C}_{\mathbb{L}}^{\text {ss }}$. 
The functor $\Xi_{\mathbb{L}, \mathbb{L}^{\prime}}$ defines an equivalence between the two gerbal representations, $\mathcal{C}_{\mathbb{L}}^{\text {ss }}$ and $\mathcal{C}_{\mathbb{L}^{\prime}}^{\text {ss }}$, that is, we have an isomorphism of functors $\Xi_{\mathbb{L}, \mathbb{L}^{\prime}} \circ F_{g} \simeq F_{g} \circ \Xi_{\mathbb{L}, \mathbb{L}^{\prime}}$ for all $g \in G L_{\infty, \infty}$ (see Definition 2.8). This follows from properties (i) and (ii) of $\Xi_{\mathbb{L}, \mathbb{L}^{\prime}}$. Thus, the gerbal representation $\mathcal{C}_{\mathbb{L}}^{\mathrm{ss}}$ is independent of the choice of the lattice $\mathbb{L} \subset \mathbb{K}$. (This is analogous to the fact that the Fock modules $M_{L}$ are isomorphic as representations of $G L_{\infty}$ for all lattices $L \subset K$.)

According to Theorem 2.10, the gerbal representation of $G L_{\infty, \infty}$ on $\mathcal{C}_{\mathbb{L}}^{\text {ss }}$ gives us a cohomology class in $H^{3}\left(G L_{\infty, \infty}, \mathcal{Z}\left(\mathcal{C}_{\mathbb{L}}^{\text {ss }}\right)^{\times}\right)$. Since $\mathcal{C}_{\mathbb{L}}^{\text {ss }}$ is $\mathbb{C}$-linear, there is a natural embedding $\mathbb{C}^{\times} \rightarrow \mathcal{Z}\left(\mathcal{C}_{\mathbb{L}}^{\mathrm{sS}}\right)^{\times}$. We will see that this class is in fact in the image $H^{3}\left(G L_{\infty, \infty}, \mathbb{C}^{\times}\right) \rightarrow H^{3}\left(G L_{\infty, \infty}, \mathcal{Z}\left(\mathcal{C}_{\mathbb{L}}^{\mathrm{ss}}\right)^{\times}\right)$, and will compute it in $\$ 5.3 .1$. (We note that this map of cohomology groups is injective since the embedding $\mathbb{C}^{\times} \rightarrow \mathcal{Z}\left(\mathcal{C}_{\mathbb{L}}^{\text {ss }}\right)^{\times}$ admits a splitting by $\mathcal{Z}\left(\mathcal{C}_{\mathbb{L}}^{\text {ss }}\right)^{\times} \rightarrow \operatorname{End}\left(M_{L}\right)^{\times} \cong \mathbb{C}^{\times}$.)

Remark 3.12. Here is an informal explanation of the above construction and the meaning of formula (3.1). Naively, we would like to identify the Clifford modules $M_{L}$ and $M_{L^{\prime}}$, where $L^{\prime}=L \cap \mathbb{L}^{\prime}$. If $L / L^{\prime}$ were a finite-dimensional subspace of $\mathbb{L} / \mathbb{L}^{\prime}$, then we would have an isomorphism

$$
M_{L} \simeq M_{L^{\prime}} \otimes \operatorname{det}\left(L / L^{\prime}\right)^{-1},
$$

sending the generating vector $|0\rangle_{L}$ to the vector (1.9) in $M_{L^{\prime}}$, which depends on the choice of a non-zero vector in $\operatorname{det}\left(L / L^{\prime}\right)$, interpreted as the wedge product of basis vectors in $L / L^{\prime}$.

But in general $L / L^{\prime}$ is infinite-dimensional, and so the Clifford modules $M_{L}$ and $M_{L^{\prime}}$ are not isomorphic, according to Lemma 3.9. But we can generalize formula (1.9) by taking the infinite wedge product of a basis in $L / L^{\prime}$. To do this, we must pick a vector in the determinant line $\Delta_{\mathbb{L}, \mathbb{L}^{\prime}}\left(L / L^{\prime}\right)$. This leads us to formula (3.1), which we use to define a gerbal action of $G L_{\infty, \infty}$ on $\mathcal{C}_{\mathbb{L}}^{\text {ss }}$. But the determinant of $L / L^{\prime}$ is non-canonical; in order to define it, we have to choose a determinantal theory $\Delta_{\mathbb{L}, \mathbb{L}^{\prime}}$ on $\mathbb{L} / \mathbb{L}^{\prime}$.

3.3. The 2-group determinantal extension of $G L_{\infty, \infty}$. In the previous section we constructed a gerbal representation of $G L_{\infty, \infty}$ on $\mathcal{C}_{\mathbb{L}}^{\text {ss }}$. This is a 2-dimensional analogue of the projective representation of $G L_{\infty}$ on the Fock representation $\wedge$ described in 1.3.3. In that case the operator on $\bigwedge=M_{L_{0}}$ corresponding to $g \in G L_{\infty}$ was defined as the composition of the tautological identification of $M_{L_{0}}$ with $M_{g L_{0}}$ and a non-trivial isomorphism $M_{g L_{0}} \simeq M_{L_{0}}$, which depends on the choice of $c \in \operatorname{det}\left(g L_{0} \mid L_{0}\right)^{\times}$. Thus, the projective action of $G L_{\infty}$ gives rise to a genuine action of $\widehat{G L}_{\infty}$, which is the $\mathbb{C}^{\times}$central extension of $G L_{\infty}$ by determinant lines. (Note that we can replace $L_{0}$ here by an arbitrary lattice $L \in K$.)

We have defined a gerbal action of $G L_{\infty, \infty}$ on the category $\mathcal{C}_{\mathbb{L}}^{\text {ss }}$ in a similar way: the functor $F_{g}, g \in G L_{\infty, \infty}$, on the category $\mathcal{C}_{\mathbb{L}}^{\text {ss }}$ is the composition of the tautological equivalence $\mathcal{C}_{\mathbb{L}}^{\mathrm{ss}} \simeq \mathcal{C}_{g \mathbb{L}}^{\mathrm{ss}}$ and a non-trivial equivalence $\Xi_{g \mathbb{L}, \mathbb{L}}: \mathcal{C}_{g \mathbb{L}}^{\mathrm{ss}} \rightarrow \mathcal{C}_{\mathbb{L}}^{\mathrm{ss}}$, which depends on the choice of a determinantal theory $\Delta_{\mathbb{L}, \mathbb{L}^{\prime}}$ on $\mathbb{L} / \mathbb{L}^{\prime}$. Hence, in order to promote this gerbal representation to a genuine representation, we must include this additional choice as part of our data. Thus, the gerbal representation of $G L_{\infty, \infty}$ gives rise to a genuine representation of a 2 -group $\mathbb{G L}_{\infty, \infty}$, which is a $B \mathbb{C}^{\times}$-central extension of $G L_{\infty, \infty}$ by determinantal gerbes which is constructed in this section. This 2-group is essentially equivalent to the 2-group construct previously by Arkhipov and Kremnizer [AK]. We will show that this extension is non-trivial, which is equivalent to the fact 
that the third cohomology class of our gerbal representation of $G L_{\infty, \infty}$ on $\mathcal{C}_{\mathbb{L}}^{\text {ss }}$ is also non-trivial.

We recall from Definition 2.1 that a 2 -group is a monoidal groupoid $\mathcal{G}$ such that the set of isomorphism classes of $\mathcal{G}$, denoted by $\pi_{0}(\mathcal{G})$, is a group under the multiplication induced from the monoidal structure.

3.3.1. Determinantal gerbes. Recall from Lemma 3.4 that if $\mathbb{L} \subset \mathbb{L}^{\prime}$ are two lattices in $\mathbb{K}$, then $\mathbb{L}^{\prime} / \mathbb{L}$ with the quotient topology is a Tate vector space. We continue to use the notation $\mathcal{D}_{\mathbb{L}^{\prime} / \mathbb{L}}$ for the $\mathbb{C}^{\times}$-gerbe of determinantal theories on $\mathbb{L}^{\prime} / \mathbb{L}$ (see Definition 1.7).

We will fix, once and for all, for each pair $\mathbb{L} \subset \mathbb{L}^{\prime}$, a linearly compact open subspace $V_{\mathbb{L}, \mathbb{L}^{\prime}} \subset \mathbb{L}^{\prime} / \mathbb{L}$, such that for any $\mathbb{L} \subset \mathbb{L}^{\prime} \subset \mathbb{L}^{\prime \prime}$, we have the exact sequence

$$
0 \rightarrow V_{\mathbb{L}, \mathbb{L}^{\prime}} \rightarrow V_{\mathbb{L}, \mathbb{L}^{\prime \prime}} \rightarrow V_{\mathbb{L}^{\prime}, \mathbb{L}^{\prime \prime}} \rightarrow 0
$$

This is always possible. For example, one can choose

$$
V_{\mathbb{L}, \mathbb{L}^{\prime}}=\mathbb{L}^{\prime} \cap \mathbb{C}[[t]]((s)) / \mathbb{L} \cap \mathbb{C}[[t]]((s)) .
$$

Recall Lemma 1.2.4. If $\mathbb{L} \subset \mathbb{L}^{\prime} \subset \mathbb{L}^{\prime \prime}$ are three lattices, then there is a canonical equivalence

$$
\mathcal{D}_{\mathbb{L}^{\prime} / \mathbb{L}} \otimes \mathcal{D}_{\mathbb{L}^{\prime \prime} / \mathbb{L}^{\prime}} \stackrel{\cong}{\cong} \mathcal{D}_{\mathbb{L}^{\prime \prime} / \mathbb{L}}
$$

(where the tensor product of two gerbes is defined in 81.8) and for a four-step filtration, there is a canonical isomorphism between two equivalences, such that for a five-step filtration, the natural diagram of these canonical isomorphisms is commutative. Now our choice $V_{\mathbb{L}^{\prime}, \mathbb{L}^{\prime \prime}}$ gives a quasi-inverse

$$
\mathcal{D}_{\mathbb{L}^{\prime \prime} / \mathbb{L}} \stackrel{\cong}{\cong} \mathcal{D}_{\mathbb{L}^{\prime} / \mathbb{L}} \otimes \mathcal{D}_{\mathbb{L}^{\prime \prime} / \mathbb{L}^{\prime}}
$$

Namely, let $\Delta_{V_{\mathbb{L}^{\prime}, \mathbb{L}^{\prime \prime}}}$ be the determinantal theory in $\mathcal{D}_{\mathbb{L}^{\prime \prime} / \mathbb{L}^{\prime}}$ such that $\Delta_{V_{\mathbb{L}^{\prime}, \mathbb{L}^{\prime \prime}}}\left(V_{\mathbb{L}^{\prime}, \mathbb{L}^{\prime \prime}}\right)=\mathbb{C}$. Then for any $\Delta \in \mathcal{D}_{\mathbb{L}^{\prime \prime} / \mathbb{L}}$ we define $\Delta^{\prime} \in \mathcal{D}_{\mathbb{L}^{\prime} / \mathbb{L}}$ as follows: let $U \subset \mathbb{L}^{\prime} / \mathbb{L}$ be a linearly compact open subspace. Choose any linearly compact open subspace $U^{\prime} \subset \mathbb{L}^{\prime \prime} / \mathbb{L}$ that contains $U$, and define $\Delta^{\prime}(U)=\Delta\left(U^{\prime}\right) \otimes \Delta_{V_{\mathbb{L}^{\prime}, \mathbb{L}^{\prime \prime}}}\left(U^{\prime} / U\right)^{-1}$. It is clear this is independent of the choice of $U^{\prime}$ up to a canonical isomorphism. Now the functor sending $\Delta \mapsto$ $\Delta^{\prime} \otimes \Delta_{V_{\mathbb{L}^{\prime}, \mathbb{L}^{\prime \prime}}}$ is the desired quasi-inverse. The fact that our choice of $\left\{V_{\mathbb{L}, \mathbb{L}^{\prime}}\right\}$ satisfies (*) makes properties similar to those of Lemma 1.8 hold for these quasi-inverses.

Now let $\mathbb{L}$ and $\mathbb{L}^{\prime}$ be two lattices in $\mathbb{K}$. Then $\mathbb{L} \cap \mathbb{L}^{\prime}$ is also a lattice. We define a $\mathbb{C}^{\times}$-gerbe

$$
\mathcal{D}\left(\mathbb{L} \mid \mathbb{L}^{\prime}\right):=\mathcal{D}_{\mathbb{L}^{\prime} / \mathbb{L} \cap \mathbb{L}^{\prime}} \otimes \mathcal{D}_{\mathbb{L} / \mathbb{L} \cap \mathbb{L}^{\prime}}^{-1} .
$$

Recall that for any gerbe $\mathcal{D}$ we denote by $\mathcal{D}^{-1}$ the dual gerbe (the objects are the same as in $\mathcal{D}$ and the set of morphisms between two objects in $\mathcal{D}^{-1}$ is the torsor dual to the set of morphisms between these objects in $\mathcal{D}$ ).

The following lemma is a consequence of Lemma 1.8 and the above discussion. In it we assume that we have made a choice of a collection of subspaces $V_{\mathbb{L}, \mathbb{L}^{\prime}} \subset \mathbb{L}^{\prime} / \mathbb{L}$ satisfying the above properties, and we use the corresponding quasi-inverse equivalences (3.2).

Lemma 3.13. (i) Any $g \in G L_{\infty, \infty}$ gives rise to an equivalence of $\mathbb{C}^{\times}$-gerbes $\mathcal{D}\left(\mathbb{L} \mid \mathbb{L}^{\prime}\right) \cong$ $\mathcal{D}\left(g \mathbb{L} \mid g \mathbb{L}^{\prime}\right)$.

(ii) There exists a preferred equivalence of categories

$$
\mathcal{D}\left(\mathbb{L} \mid \mathbb{L}^{\prime}\right) \otimes \mathcal{D}\left(\mathbb{L}^{\prime} \mid \mathbb{L}^{\prime \prime}\right) \cong \mathcal{D}\left(\mathbb{L} \mid \mathbb{L}^{\prime \prime}\right)
$$


and natural transformations between the two equivalences

$$
\mathcal{D}\left(\mathbb{L}_{1} \mid \mathbb{L}_{2}\right) \otimes \mathcal{D}\left(\mathbb{L}_{2} \mid \mathbb{L}_{3}\right) \otimes \mathcal{D}\left(\mathbb{L}_{3} \mid \mathbb{L}_{4}\right) \cong \mathcal{D}\left(\mathbb{L}_{1} \mid \mathbb{L}_{4}\right)
$$

such that the diagram of natural transformations is commutative.

3.3.2. $B \mathbb{C}^{\times}$-central extension of $G L_{\infty, \infty}$. Let $\mathcal{G}$ be a 2-group. In Definition 2.5 we introduced the notion of central extension of $\pi_{0}(\mathcal{G})$ by $B \pi_{1}(\mathcal{G})$.

Now we define a $B \mathbb{C}^{\times}$-central extension of $G L_{\infty, \infty}$. We fix a lattice $\mathbb{L} \subset \mathbb{K}$. Let $\mathbb{G L}_{\infty, \infty}$ be the following category: objects are $(g, \Delta), g \in G L_{\infty, \infty}, \Delta \in \mathcal{D}(g \mathbb{L} \mid \mathbb{L})$; the set of morphisms $\operatorname{Hom}_{\mathbb{G L}_{\infty}, \infty}\left((g, \Delta),\left(g^{\prime}, \Delta^{\prime}\right)\right)$ is empty if $g \neq g^{\prime}$ and is the space $\operatorname{Hom}_{\mathcal{D}(g \mathbb{L} \mid \mathbb{L})}\left(\Delta, \Delta^{\prime}\right)$ if $g=g^{\prime}$. We define the monoidal structure

$$
m: \mathbb{G L}_{\infty, \infty} \times \mathbb{G L}_{\infty, \infty} \rightarrow \mathbb{G L}_{\infty, \infty}
$$

by $m\left((g, \Delta),\left(g^{\prime}, \Delta^{\prime}\right)\right)=\left(g g^{\prime}, \Delta \otimes g\left(\Delta^{\prime}\right)\right)$. Here we regard $\Delta \otimes g\left(\Delta^{\prime}\right)$ as an object of the category

$$
\mathcal{D}(g \mathbb{L} \mid \mathbb{L}) \otimes \mathcal{D}\left(g g^{\prime} \mathbb{L} \mid g \mathbb{L}\right) \cong \mathcal{D}\left(g g^{\prime} \mathbb{L} \mid \mathbb{L}\right),
$$

using Lemma 3.13. This Lemma also guarantees that $\mathbb{G L}_{\infty, \infty}$ is a 2-group which is a monoidal category. It is clear that $\pi_{1}\left(\mathbb{G} \mathbb{L}_{\infty, \infty}\right)=\mathbb{C}^{\times}$and the action of $\pi_{0}\left(\mathbb{G} \mathbb{L}_{\infty, \infty}\right)=$ $G L_{\infty, \infty}$ on $\pi_{1}\left(\mathbb{G L}_{\infty, \infty}\right)$ is trivial. Thus, $\mathbb{G L}_{\infty, \infty}$ is a $B \mathbb{C}^{\times}$-central extension of $G L_{\infty, \infty}$.

3.3.3. The genuine action of $\mathbb{G L}_{\infty, \infty}$ on $\mathcal{C}_{\mathbb{L}}^{\mathrm{ss}}$. Now we show that the gerbal representation of $G L_{\infty, \infty}$ gives rise to a genuine representation of the 2-group $\mathbb{G L}_{\infty, \infty}$.

Let $\mathbb{L}$ and $\mathbb{L}^{\prime}$ be two lattices of $\mathbb{K}$. We claim that there is a functor

$$
\Xi: \mathcal{D}\left(\mathbb{L} \mid \mathbb{L}^{\prime}\right) \rightarrow \mathbb{F} U \mathbb{N} \mathbb{C}\left(\mathcal{C}_{\mathbb{L}}^{\text {ss }}, \mathcal{C}_{\mathbb{L}^{\prime}}^{\text {ss }}\right)
$$

where $\mathbb{F} U \mathbb{N} \mathbb{C}\left(\mathcal{C}_{\mathbb{K} / \mathbb{L}}^{\text {ss }}, \mathcal{C}_{\mathbb{K} / \mathbb{L}^{\prime}}^{\text {ss }}\right)$ denotes the category of additive functors between these two abelian categories, such that the following diagram commutes up to a canonical isomorphism.

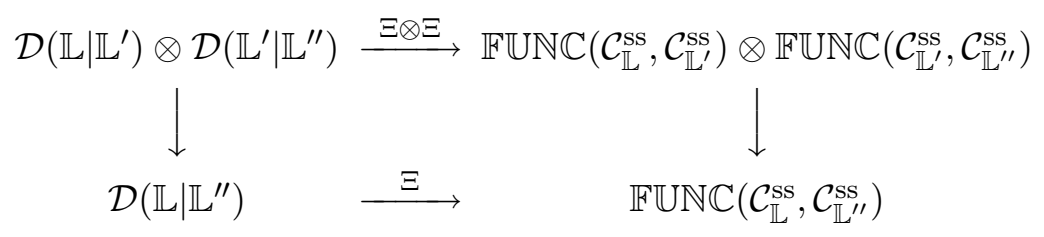

It is clear that we need only to consider the case $\mathbb{L} \subset \mathbb{L}^{\prime}$ and $\mathbb{L} \supset \mathbb{L}^{\prime}$. We first consider the case $\mathbb{L} \supset \mathbb{L}^{\prime}$. Then $\mathcal{D}\left(\mathbb{L} \mid \mathbb{L}^{\prime}\right)=\mathcal{D}_{\mathbb{L} / \mathbb{L}^{\prime}}^{-1}$. An object $\Delta$ is a determinantal theory of the Tate vector space $\mathbb{L} / \mathbb{L}^{\prime}$. If $L \subset \mathbb{L}$ is a secondary lattice, then $L \cap \mathbb{L}^{\prime}$ is a secondary lattice of $\mathbb{L}^{\prime}$ and $L /\left(L \cap \mathbb{L}^{\prime}\right)$ is a linearly compact open subspace of $\mathbb{L} / \mathbb{L}^{\prime}$. Now $\Xi$ is defined on objects by the formula

$$
\Xi(\Delta)\left(M_{L}\right)=M_{L \cap \mathbb{L}^{\prime}} \otimes \Delta\left(L /\left(L \cap \mathbb{L}^{\prime}\right)\right)^{-1},
$$

We define $\Xi(\Delta)$ on morphisms so as to make $\Xi(\Delta) \in \mathbb{F} \mathbb{U N} \mathbb{C}\left(\mathcal{C}_{\mathbb{K} / \mathbb{L}}^{\text {ss }}, \mathcal{C}_{\mathbb{K} / \mathbb{L}^{\prime}}^{\text {ss }}\right)$ in the same way as in the proof of Theorem 3.11. Therefore, $\Xi$ defines a functor from $\mathcal{D}\left(\mathbb{L} \mid \mathbb{L}^{\prime}\right)$ to $\mathbb{F} U \mathbb{N} \mathbb{C}\left(\mathcal{C}_{\mathbb{K} / \mathbb{L}}^{\mathrm{ss}}, \mathcal{C}_{\mathbb{K} / \mathbb{L}^{\prime}}^{\mathrm{ss}}\right)$.

Next, we consider the case $\mathbb{L} \subset \mathbb{L}^{\prime}$. Then $\mathcal{D}\left(\mathbb{L} \mid \mathbb{L}^{\prime}\right)=\mathcal{D}_{\mathbb{L}^{\prime} / \mathbb{L}}$. Let $\Delta \in \mathcal{D}_{\mathbb{L}^{\prime} / \mathbb{L}}$. For any secondary lattice $L \subset \mathbb{L}$ we define

$$
\Xi(\Delta)\left(M_{L}\right)=M_{L^{\prime}} \otimes \Delta\left(V_{\mathbb{L}, \mathbb{L}^{\prime}}\right),
$$

where $L^{\prime}$ is any secondary lattice in $\mathbb{L}^{\prime}$ fitting into the following exact sequence

$$
0 \rightarrow L \rightarrow L^{\prime} \rightarrow V_{\mathbb{L}, \mathbb{L}^{\prime}} \rightarrow 0
$$


Observe that $M_{L^{\prime}}$ is independent of the choice of $L^{\prime}$ up to a canonical isomorphism. So $\Xi$ is well-defined in this case.

Now we define the genuine action of the 2-group $\mathbb{G L}_{\infty, \infty}$ on $\mathcal{C}_{\mathbb{L}}^{\text {ss }}$ as follows: for $(g, \Delta) \in \mathbb{G L}_{\infty, \infty}, F_{(g, \Delta)}: \mathcal{C}_{\mathbb{L}}^{\mathrm{sS}} \rightarrow \mathcal{C}_{\mathbb{L}}^{\text {ss }}$ is defined as the composition

$$
\mathcal{C}_{\mathbb{L}}^{\mathrm{ss}} \stackrel{g}{\rightarrow} \mathcal{C}_{g \mathbb{L}}^{\mathrm{ss}} \stackrel{\Xi(\Delta)}{\rightarrow} \mathcal{C}_{\mathbb{L}}^{\mathrm{ss}}
$$

3.4. 2-infinite Grassmannian. This subsection is completely independent from the rest of the paper. Here we discuss informally a possible geometric realization of the gerbal representation constructed in the previous section.

First, let us recall the 1-dimensional story. Consider the set of lattices in the Tate vector space $K=\mathbb{C}((t))$. Let us pick a lattice, for instance, $L_{0}=\mathbb{C}[[t]]$. Then to any lattice $L \in K$ we associate a line $\operatorname{det}\left(L \mid L_{0}\right)$ and a $\mathbb{C}^{\times}$-torsor $\operatorname{det}\left(L \mid L_{0}\right)^{\times}$. The group $G L_{\infty}$ acts transitively on the set of lattices, but this action does not lift to the union of these $\mathbb{C}^{\times}$-torsors. In fact, what lifts is a non-trivial central extension $\widehat{G L}_{\infty}$ of $G L_{\infty}$. This is clear from the definition of this central extension given in $\$ 1.2 .3$ (as the group $\left.\widehat{G L}_{\infty}^{\prime}\right)$.

We can use these geometric objects to construct a projective representation of $G L_{\infty}$ on a vector space. For this we present the set of lattices as the set of $\mathbb{C}$-points of an ind-scheme $\mathrm{Gr}$ (see 1.2 .1 ) and the set of torsors as the set of $\mathbb{C}$-points of a principal $\mathbb{G}_{m}$-bundle $\mathcal{L}^{\times}$on Gr. Here $\mathcal{L}$ is the determinant line bundle introduced in $\$ 1.2 .2$, Now we can take the space of global sections of $\mathcal{L}^{*}$, and the corresponding dual space is the Fock representation $\bigwedge$ of $\widehat{G L}_{\infty}$ (see formula (1.8) $)$.

We would like to imitate this in the 2-dimensional case. The set-theoretic part of the story is straightforward (see also [AK] ). We have the set of lattices in $\mathbb{K}$, and the group $G L_{\infty, \infty}$ acts transitively on it. Let us pick a lattice, for instance, $\mathbb{L}_{0}=\mathcal{O}_{\mathbb{K}}$. Then to any lattice $\mathbb{L}$ we associate the $\mathbb{C}^{\times}$-gerbe $\mathcal{D}\left(\mathbb{L} \mid \mathbb{L}_{0}\right)$. The action of $G L_{\infty, \infty}$ on the set of lattices lifts to an action of the 2-group $\mathbb{G L}_{\infty, \infty}$ on these gerbes. If we could realize these objects algebro-geometrically, then we would be able to construct a representation of $\mathbb{G} \mathbb{L}_{\infty, \infty}$ (that is, a gerbal representation of $G L_{\infty, \infty}$ ) on the corresponding category of "global sections". At the moment, we do not know how to do this, but here are some indications of how this could be done.

For a Tate vector space $V$ with a countable basis of neighborhoods of 0 , we will denote by $\operatorname{Gr}(V)$ the moduli space of closed Tate vector subspaces of $V$. The corresponding functor from commutative $\mathbb{C}$-algebras to sets is defined as follows: for any commutative $\mathbb{C}$-algebra $R$,

$$
\operatorname{Gr}(V)(R)=\{\text { Tate } R \text {-modules that are direct summands of } R \widehat{\otimes} V\} \text {. }
$$

Therefore, a map $\operatorname{Spec} R \rightarrow \operatorname{Gr}(V)$ is equivalent to a Tate $R$-module (see [Dr], §3.2.1 for the definition of Tate $R$-modules), which is a direct summand of $R \widehat{\otimes} V$. One can show that $\operatorname{Gr}(V)$ is a $\mathbb{C}$-space. Observe that for $V=K$ the infinite Grassmannian Gr defined in $\$ 1.2 .1$ is a subspace of $\operatorname{Gr}(K)$. While the later consists of all closed Tate vector subspaces of $K$, the former only consists of those which are linearly compact.

If $M$ is a Tate $R$-module, then there is a $\mathbb{G}_{m}$-gerbe over $\operatorname{Spec} R$ in Nisnevich topology, namely, the gerbe $\mathcal{D}_{M}$ of determinantal theories of $M$ (see [Dr], §3.6). Therefore, there is a tautological $\mathbb{G}_{m}$-gerbe over $\operatorname{Gr}(V)$, which assigns to any $u: \operatorname{Spec} R \rightarrow \operatorname{Gr}(V)$ the gerbe of determinantal theories of the Tate $R$-module corresponding to $u$. Denote this $\mathbb{G}_{m}$-gerbe over $\operatorname{Gr}(V)$ by $\mathcal{D}_{\mathrm{Gr}(V)}$. 
Now denote by $2 \mathrm{Gr}$ the sought-after moduli of lattices of $\mathbb{K}=\mathbb{C}((t))((s))$. It is natural to define it as the direct limit

$$
2 \mathrm{Gr}=\lim _{\vec{N}} \operatorname{Gr}\left(s^{-N} \mathcal{O}_{\mathbb{K}} / s^{N} \mathcal{O}_{\mathbb{K}}\right) .
$$

The tautological gerbes over these spaces should be compatible with the pull-backs under the embeddings

$$
\operatorname{Gr}\left(s^{-N} \mathcal{O}_{\mathbb{K}} / s^{N} \mathcal{O}_{\mathbb{K}}\right) \rightarrow \operatorname{Gr}\left(s^{-N-1} \mathcal{O}_{\mathbb{K}} / s^{N+1} \mathcal{O}_{\mathbb{K}}\right),
$$

and therefore we should obtain a $\mathbb{G}_{m}$-gerbe $\mathcal{D}_{2 \mathrm{Gr}}$ over $2 \mathrm{Gr}$.

Alternatively, consider the Cartesian square $2 \mathrm{Gr} \times 2 \mathrm{Gr}$. Then there should be a tautological $\mathbb{G}_{m}$-gerbe over it, whose fiber over $\left(\mathbb{L}, \mathbb{L}^{\prime}\right)$ is the gerbe $\mathcal{D}\left(\mathbb{L} \mid \mathbb{L}^{\prime}\right)$ as defined in 3.3.1, The restriction of this gerbe to $2 \mathrm{Gr} \times\left\{\mathcal{O}_{\mathbb{K}}\right\}$ is the above gerbe $\mathcal{D}_{2 \mathrm{Gr}}$.

The group $G L_{\infty, \infty}$ acts transitively on the set of $\mathbb{C}$-points of $2 \mathrm{Gr}$ (see Lemma 3.5), and this action lifts to a tautological action of $\mathbb{G L}_{\infty, \infty}$ (see 33.3 .2 ) on $\mathcal{D}_{2 \mathrm{Gr}}$.

Recall that for a $\mathbb{G}_{m}$-gerbe $\mathcal{G}$ over a scheme $S$, there is a notion of a $\mathcal{G}$-twisted $\mathcal{O}$-module on $S$ (see, e.g., [Li]). We denote the category of $\mathcal{G}$-twisted $\mathcal{O}$-modules on $S$ by $\operatorname{QCoh}_{S}(\mathcal{G})$. The $\mathbb{G}_{m}$-gerbe $\mathcal{G}$ also gives rise to a sheaf of abelian categories over $S, \mathcal{C}:=\mathcal{G} \underset{B \mathbb{G}_{m}}{\times}$ Vect, and $\operatorname{QCoh}_{S}(\mathcal{G})$ may be viewed as the category of global sections of $\mathcal{C}$. Since there is a an action of $\mathbb{G L}_{\infty, \infty}$ on $\mathcal{D}_{2 \mathrm{Gr}}$, we should obtain a (genuine) representation of $\mathbb{G L}_{\infty, \infty}$ (and hence a gerbal representation of $G L_{\infty, \infty}$ ) on $\mathrm{QCoh}_{2 \mathrm{Gr}}\left(\mathcal{D}_{2 \mathrm{Gr}}\right)$. In addition, we expect that there is a $G L_{\infty, \infty}$-equivariant embedding of categories $\mathcal{C}_{\mathcal{O}_{\mathbb{K}}}^{\text {ss }} \rightarrow \mathrm{QCoh}_{2 \mathrm{Gr}}\left(\mathcal{D}_{2 \mathrm{Gr}}\right)$.

This is still a very rough scenario in which many details need to be worked out. But let us look at the following simplified version, which is its 1-dimensional analogue. Let $V$ be again a Tate vector space, with a countable basis of neighborhoods of 0 . Let $\operatorname{Gr}(V)$ be the moduli space of closed Tate vector subspaces of $V$ as defined in (3.3) and $\mathcal{D}_{\mathrm{Gr}(V)}$ the tautological $\mathbb{G}_{m}$-gerbe over it. Let $G L(V)$ be the group of continuous automorphisms of $V$. Note that $G L(V)=G L_{\infty}$ if $V=K$. Now $G L(V)$ acts (genuinely) on $\operatorname{Gr}(V)$, as well as on $\mathcal{D}_{\operatorname{Gr}(V)}$. Therefore, there is a genuine representation of $G L(V)$ on $\mathrm{QCoh}_{\mathrm{Gr}(V)}\left(\mathcal{D}_{\mathrm{Gr}(V)}\right)$. On the other hand, we recall that $\mathcal{C}_{V}$ is the category of discrete Clifford modules over $\mathrm{Cl}_{V}$ (see $\$ 1.3 .3$ ), and there is a genuine representation of $G L(V)$ on $\mathcal{C}_{V}$ ( since $G L(V)$ acts by automorphisms of $\mathrm{Cl}_{V}$ ). We claim that there is a $G L(V)$ equivariant embedding $\mathcal{C}_{V} \rightarrow \mathrm{QCoh}_{\mathrm{Gr}(V)}\left(\mathcal{D}_{\mathrm{Gr}(V)}\right)$.

To see that, we first need a strengthening of Lemma 1.10, which can be proven using the results in $\mathrm{BBE}, \S 2.14-2.15$. Let $R$ be a commutative ring and $M$ a Tate $R$-module. Consider the Clifford algebra $\mathrm{Cl}_{M}=\mathrm{Cl}_{R}\left(M \oplus M^{*}\right)$ and denote by $\mathcal{C}_{M}$ the abelian category of discrete modules of $\mathrm{Cl}_{M}$. Let $\mathcal{D}_{M}$ be the $\mathbb{G}_{m}$-gerbe of determinantal theories of $M$ in Nisnevich topology on $\operatorname{Spec} R$. We have the following

Proposition 3.14. The category $\mathcal{C}_{M}$ is naturally equivalent to the category of $\mathcal{D}_{M^{-}}$ twisted $\mathcal{O}$-modules over $\operatorname{Spec} R$.

Now we will associate to every lattice $L \subset V$ of $V$ an object $\mathcal{F}_{L} \in \mathrm{QCoh}_{\mathrm{Gr}(V)}\left(\mathcal{D}_{\mathrm{Gr}(V)}\right)$. Let $u: \operatorname{Spec} R \rightarrow \operatorname{Gr}(V)$ be a morphism given by $M \subset R \widehat{\otimes} V$. For any $L$, the tensor product $R \widehat{\otimes} L$ is a lattice in $R \widehat{\otimes} V$, and $L_{M}:=(R \widehat{\otimes} L) \cap M$ is a lattice in $M$. Let $M_{L_{M}}$ be the vacuum module over $\mathrm{Cl}_{M}$ induced from the trivial representation of $\bigwedge\left(L_{M} \oplus L_{M}^{\perp}\right)$. By the above proposition, we obtain an object in $\mathrm{QCoh}_{\mathrm{Spec} R}\left(\mathcal{D}_{M}\right)$, which we denote 
by $u^{*} \mathcal{F}_{L}$. Then the collection $\left\{u^{*} \mathcal{F}_{L}\right\}_{u: \operatorname{Spec} R \rightarrow \operatorname{Gr}(V)}$ define the desired object $\mathcal{F}_{L} \in$ $\mathrm{QCoh}_{\mathrm{Gr}(V)}(\operatorname{Gr}(V))$.

Finally, there is a $G L(V)$-equivariant functor $\mathcal{C}_{V} \rightarrow \mathrm{QCoh}_{\mathrm{Gr}(V)}\left(\mathcal{D}_{\mathrm{Gr}(V)}\right)$ that will send $M_{L}$ to $\mathcal{F}_{L}$, where $M_{L} \in \mathcal{C}_{V}$ is the vacuum module of $\mathrm{Cl}_{V}$ associated with $L$.

Thus, we see that a simplified version of our proposal does work.

\section{Cohomology of $G L_{\infty, \infty}$ And Related groups}

In the previous section we have constructed a gerbal representation of the group $G L_{\infty, \infty}$ on a certain category of modules over a Clifford algebra. It is important to identify the third cohomology class arising in this gerbal representation. In this section, we first discuss the relevant cohomology groups. In the next section we will show that there exists of a particular non-zero cohomology class $\left[E_{3}\right] \in H^{3}\left(G L_{\infty, \infty}, \mathbb{C}^{\times}\right)$and this class corresponds to the gerbal representation of $G L_{\infty, \infty}$ on $\mathcal{C}_{\mathcal{O}_{\mathbb{K}}}^{\mathrm{ss}}$ constructed in Theorem 3.11.

Analogous results on the cohomologies of the Lie algebra $\mathfrak{g l}_{\infty, \infty}$ and related Lie algebras will be discussed in [FZ].

\subsection{Lie algebras and Lie groups of matrices.}

4.1.1. Lie algebras of matrices. Let $R$ be an associative (not necessarily unital) $\mathbb{C}$ algebra. By definition, $R$ is a $\mathbb{C}$-vector space, with a ring structure such that the multiplication $R \times R \rightarrow R$ is $\mathbb{C}$-bilinear.

We will denote by $R((t))$ the $\mathbb{C}$-algebra of Laurent series with coefficients in $R$. We will just regard it as a topological right $R$-module, endowed with the $t$-adic topology. Let $R[[t]]$ denote the $\mathbb{C}$-algebra of power series with coefficients in $R$. Then it is an open submodule of $R((t))$. Observe that we have the following polarization:

$$
R((t))=R[[t]] \oplus t^{-1} R\left[t^{-1}\right] .
$$

Recall that we define $\mathfrak{g l}_{\infty}(-)$ as a functor from the category of associative (not necessarily unital) $\mathbb{C}$-algebras to itself by assigning to any $\mathbb{C}$-algebra $R$ the algebra of continuous right $R$-module endomorphisms of $R\left((t)\right.$ ) (so that $\mathfrak{g l}_{\infty}(R)$ acts on $R((t)$ ) from the left). Likewise, we define $\mathfrak{g l}_{\infty}^{+}(R)$ as the algebra of continuous right $R$-module endomorphisms of $R[[t]]$, and define $\mathfrak{g l}_{\mathfrak{f}}(R)$ to be the two-sided ideal of $\mathfrak{g l}_{\infty}^{+}(R)$ consisting of discrete $R$-module endomorphisms of $R[[t]]$, i.e., endomorphisms $f: R[[t]] \rightarrow R[[t]]$ such that $\exists N,\left.f\right|_{t^{N} R[[t]]}=0$.

Finally, there is a natural map $\pi: \mathfrak{g l}_{\infty}(R) \rightarrow \mathfrak{g l}_{\infty}^{+}(R)$ (not an algebra homomorphism) induced by the natural projection $\pi: R((t)) \rightarrow R[[t]]$ with respect to the decomposition $R((t))=R[[t]] \oplus t^{-1} R\left[t^{-1}\right]$. Namely, $\pi(A) \in \mathfrak{g l}_{\infty}^{+}(R)$ is defined by the formula

$$
\pi(A) x=\pi(A x) \quad \text { for } x \in R[[t]]
$$

Now we generalize the construction in $\$ 1$ as follows. Define

$$
\tilde{\mathfrak{g l}}_{\infty}(R)=\left\{(A, X) \in \mathfrak{g l}_{\infty}^{+}(R) \times \mathfrak{g l}_{\infty}(R), A-\pi(X) \in \mathfrak{g l}_{\mathfrak{f}}(R)\right\}
$$

One has the following exact sequence of associative algebras (and therefore Lie algebras)

$$
0 \rightarrow \mathfrak{g l}(R) \stackrel{i}{\rightarrow} \widetilde{\mathfrak{g l}}_{\infty}(R) \stackrel{p}{\rightarrow} \mathfrak{g l}_{\infty}(R) \rightarrow 0
$$

where $i(A)=(A, 0)$ and $p(A, X)=X$. One also has the section of $p$ given by $X \mapsto$ $(\pi(X), X)$. 
Therefore, $H_{*}(\mathfrak{g l}(R)), H_{*}\left(\mathfrak{g l}_{\infty}^{+}(R)\right), H_{*}\left(\mathfrak{g l}_{\infty}(R)\right)$ have the structures of graded Hopf algebras.

4.1.2. Groups of matrices. Likewise, we will define $G L_{\infty}(R)$ to be the group of continuous right $R$-module automorphisms of $R((t)), G L_{\infty}^{+}(R)$ the group of continuous right $R$-module automorphisms of $R[[t]]$, and $G L_{\mathfrak{f}}$ the normal subgroup of $G L_{\infty}^{+}(R)$ consisting of those $g$ such that $\exists N,\left.g\right|_{t^{N} R[[t]]}=$ id. Furthermore, let $\widetilde{G L}_{\infty}(R)$ be the group of invertible elements in $\widetilde{\mathfrak{g l}}_{\infty}(R)$, defined in the previous subsection. Unlike the case of Lie algebras where we have the short exact sequence (4.1), for groups we only have the following sequence

$$
1 \rightarrow G L_{\mathfrak{f}}(R) \stackrel{i}{\rightarrow} \widetilde{G L}_{\infty}(R) \stackrel{p}{\rightarrow} G L_{\infty}(R),
$$

where $i: G L_{\mathfrak{f}}(R) \rightarrow \widetilde{G L}_{\infty}(R)$ is given by $i(a)=(a, 1)$. (We recall that $p(a, g)=g$.) We will have a detailed discussion of this sequence in $\$ 4.2 .2$,

If $R$ has the unit, and if we use the standard topological basis $\left\{t^{i}\right\}$ of $R((t))$, these groups can be written in the following concrete terms:

$$
\begin{gathered}
G L_{\mathfrak{f}}(R)=\left\{\left(a_{i j}\right)_{i, j \geq 0}, a \text { invertible }, a_{i j} \in R, a_{i j}=\delta_{i j} \text { for } j \gg 0\right\}, \\
G L_{\infty}^{+}(R)=\left\{\begin{array}{l}
\left(a_{i j}\right)_{i, j \geq 0}, a \text { invertible }, a_{i j} \in R \mid \forall m \geq 0, \exists n \geq 0, \\
\text { such that whenever } i<m, j>n, a_{i j}=0
\end{array}\right\}, \\
G L_{\infty}(R)=\left\{\begin{array}{l}
\left(a_{i j}\right)_{i, j \in \mathbb{Z}}, a \text { invertible }, a_{i j} \in R \mid \forall m \in \mathbb{Z}, \exists n \in \mathbb{Z}, \\
\text { such that whenever } i<m, j>n, a_{i j}=0
\end{array}\right\} .
\end{gathered}
$$

The left actions of these group on $R((t))$ are given by $a t^{j}=\sum a_{i j} t^{i}$.

Remark 4.1. Even if $R$ does not have the unit, we can still present these groups in matrix forms. For example, $G L_{\mathfrak{f}}(R)$ consists of those $A=\left(A_{i j}\right)_{i, j \geq 0}, A_{i j}=0$ for $j \gg 0$, such that there exists some $B, A+B+A B=0$, where $A B$ is the usual matrix multiplication.

Observe that $G L(R), G L_{\infty}^{+}(R), G L_{\infty}(R)$ have inner sums, that is, group homomorphisms

$$
\begin{aligned}
& \oplus: G L(R) \times G L(R) \rightarrow G L(R) \\
& \oplus: G L_{\infty}^{+}(R) \times G L_{\infty}^{+}(R) \rightarrow G L_{\infty}^{+}(R) \\
& \oplus: G L_{\infty}(R) \times G L_{\infty}(R) \rightarrow G L_{\infty}(R)
\end{aligned}
$$

defined as follows: for $a=\left(a_{i j}\right), b=\left(b_{i j}\right), a \oplus b=c$, where

$$
c_{i j}= \begin{cases}a_{\frac{i}{2}, \frac{j}{2}} & \text { if } i, j \text { even }, \\ b_{\frac{i-1}{2}, \frac{j-1}{2}} & \text { if } i, j \text { odd }, \\ 0 & \text { otherwise }\end{cases}
$$

Let us remark that $\mathfrak{g l}_{\mathfrak{f}}(R), \mathfrak{g l}_{\infty}^{+}(R)$ and $\mathfrak{g l}_{\infty}(R)$ also have inner sums, given by the same formula as in the previous subsection.

4.1.3. Notation. As in $₫ \mathbb{1}$, for brevity, $\mathfrak{g l}_{\mathfrak{f}}(\mathbb{C}), \mathfrak{g l}_{\infty}^{+}(\mathbb{C}), \mathfrak{g l}_{\infty}(\mathbb{C})$ and $\tilde{\mathfrak{g l}}_{\infty}(\mathbb{C})$ will be denoted by $\mathfrak{g l}_{\mathfrak{f}}, \mathfrak{g l}_{\infty}^{+}, \mathfrak{g l}_{\infty}$, and $\widetilde{\mathfrak{g l}}_{\infty}$, respectively. Likewise, $G L_{\mathfrak{f}}(\mathbb{C}), G L_{\infty}^{+}(\mathbb{C}), G L_{\infty}(\mathbb{C})$ and $\widetilde{G L}(\mathbb{C})$ will be denoted by $G L_{\mathfrak{f}}, G L_{\infty}^{+}, G L_{\infty}$ and $\left.\widetilde{G L}{ }_{\infty}\right)$, respectively. As in $\oint_{3}$, we denote $G L_{\infty}\left(\mathfrak{g l}_{\infty}\right)$ by $G L_{\infty, \infty}$. Furthermore, we will denote $G L_{\mathfrak{f}}\left(\mathfrak{g l}_{\infty}\right)$ by $G L_{\mathfrak{f}, \infty}$. 
4.2. Computation of group cohomology. We do not have a complete description of the cohomology for the groups introduced above (unlike the Lie algebra case, which will be treated in [FZ). However, we still obtain some interesting cohomology classes that will be sufficient for our purposes.

4.2.1. Starting point. We do not know the full cohomology groups $H^{\bullet}\left(G L_{\mathfrak{f}}, \mathbb{C}^{\times}\right)$. However, the determinant det $: G L_{\mathfrak{f}} \rightarrow \mathbb{C}^{\times}$determines a class [det] $\in H^{1}\left(G L_{\mathfrak{f}}, \mathbb{C}^{\times}\right)$, which is the starting point of the following construction.

Next, we turn to $G L_{\infty}$ and $G L_{\infty, \infty}$. Recall the definition of $\widetilde{G L}_{\infty}(R)$ from $\$ 4.1 .2$. We have

Proposition 4.2. For any $\mathbb{C}$-algebra $R, H^{\bullet}\left(G L_{\infty}^{+}(R), \mathbb{C}^{\times}\right)=H^{\bullet}\left(\widetilde{G L}_{\infty}(R), \mathbb{C}^{\times}\right) \cong \mathbb{C}^{\times}$.

Proof. Let us in fact prove that $H_{*}\left(G L_{\infty}^{+}(R), k\right)=H_{*}\left(\widetilde{G L}_{\infty}(R), k\right) \cong k$ for any field $k$. The proposition then follows from the universal coefficient theorem.

We first prove that $H_{*}\left(G L_{\infty}^{+}(R), k\right) \cong k$. This is in fact proved in [W]. For the sake of completeness, we reproduce the proof here.

Recall that $H_{*}\left(G L_{\infty}^{+}(R), k\right)$ has the structure of a Hopf algebra since we have the inner sum " $\oplus$ ". We will show that there is a group homomorphism $\tau: G L_{\infty}^{+}(R) \rightarrow$ $G L_{\infty}^{+}(R)$ such that for any $a \in G L_{\infty}^{+}(R), a \oplus \tau(a)=\tau(a)$. Using this fact, we prove $H_{*}\left(G L_{\infty}^{+}(R), k\right) \cong k$ by induction. Assume that $H_{n-1}\left(G L_{\infty}^{+}(R), k\right)=0$. Then for $x \in H_{n}\left(\mathfrak{g l}_{\infty}^{+}(R), k\right), \Delta(x)=x \otimes 1+1 \otimes x+\sum u_{i} \otimes v_{i}$, where $u_{i}, v_{i} \in H_{k}\left(G L_{\infty}^{+}(R)\right), k=$ $1,2, \ldots, n-1$. By induction, $u_{i}, v_{i}$ vanish and therefore, $\Delta(x)=x \otimes 1+1 \otimes x$. Since

$$
\tau=\oplus \circ(\mathrm{id} \oplus \tau) \circ \Delta: G L_{\infty}^{+}(R) \rightarrow G L_{\infty}^{+}(R)
$$

one obtain that $\tau_{*}(x)=x+\tau_{*}(x)$ and therefore $x=0$.

Now we construct the morphism $\tau$ : for $a=\left(a_{i j}\right) \in G L_{\infty}^{+}(R)$, we set

$$
\tau(a)_{i j}= \begin{cases}a_{i / 2, j / 2} & \text { if } i, j \text { even; } \\ a_{m, n} & \text { if } i=2^{k} m+2^{k-1}-1, j=2^{k} n+2^{k-1}-1 \\ 0 & \text { otherwise }\end{cases}
$$

It is easy to check that $\tau$ has the required properties.

Next, we show that $H_{*}\left(\widetilde{G L}_{\infty}(R), k\right) \cong k$. We have the surjective group homomorphism

$$
1 \rightarrow J(R) \rightarrow \widetilde{G L}_{\infty}(R) \rightarrow G L_{\infty}^{+}(R) \rightarrow 1
$$

sending $(a, g) \rightarrow a$. The kernel is

$$
J(R)=\left\{g=\left(g_{i j}\right)_{i, j \in \mathbb{Z}} \in G L_{\infty}(R), g_{i j}=\delta_{i j} \text { for } j \gg 0\right\}
$$

Therefore, it is enough to prove that $H_{*}(J(R), k) \cong k$. We could present $J(R)$ as

$$
J(R)=\lim _{\vec{n}} J_{n}(R)
$$

where

$$
J_{n}(R)=\left\{g=\left(g_{i j}\right)_{i, j \in \mathbb{Z}} \in G L_{\infty}(R), g_{i j}=\delta_{i j} \text { for } j \gg n\right\}
$$

Observe that all $J_{n}(R)$ is indeed isomorphic to $J_{0}(R)$, and therefore it is enough to show that $H_{*}\left(J_{0}(R), k\right) \cong k$. This in fact follows from the same argument as for $G L_{\infty}^{+}(R)$. 
4.2.2. Two exact sequences of groups. Recall that from 4.1 .2 we have a left exact sequence of groups

$$
1 \rightarrow G L_{\mathfrak{f}}(R) \stackrel{i}{\rightarrow} \widetilde{G L}_{\infty}(R) \stackrel{p}{\rightarrow} G L_{\infty}(R) .
$$

In general, the map $p: \widetilde{G L}_{\infty}(R) \rightarrow G L_{\infty}(R)$ is not surjective. We have the following:

Proposition 4.3. There is a well-defined surjective group homomorphism

$$
\operatorname{deg}: G L_{\infty}(R) \rightarrow K_{0}\left(R^{\mathrm{op}}\right)
$$

where $K_{0}\left(R^{\mathrm{op}}\right)$ is the Grothendieck group of the category of finitely generated projective right $R$-modules.

Proof. The existence of deg : $G L_{\infty}(R) \rightarrow K_{0}\left(R^{\mathrm{op}}\right)$ and its surjectivity can be proved in a way similar to [FW] Proposition 1.3. We need the following

Lemma 4.4. For any $g \in G L_{\infty}(R)$, and any integer $M$, there exists an integer $N$ such that $t^{M} R[[t]] \cdot g \supset t^{N} R[[t]]$ and $\frac{t^{M} R[[t]] \cdot g}{\left.t^{N} R[t]\right]}$ is a finitely generated projective $R$-module. (Then any $N^{\prime} \geq N$ also satisfies this property.)

Proof. Since $g \in G L_{\infty}(R)$ is continuous, for any $m$ there exists $n$ such that $t^{m} R[[t]] \supset$ $g t^{n} R[[t]]$. Therefore, for a given $M$, we have

$$
g t^{M} R[[t]] \supset t^{n_{1}} R[[t]] \supset g t^{n_{2}} R[[t]] \supset t^{n_{3}} R[[t]]
$$

We claim that $N=n_{3}$ satisfies the desired property. Indeed, $\frac{g t^{M} R[[t]]}{\left.t^{n} R[t]\right]}$ has projective dimension one (i.e., $\operatorname{Ext}^{i}\left(\frac{g t^{M} R[[t]]}{\left.t^{n_{1}} R[t]\right]},-\right)=0$ for $i \geq 2$ ) since $\frac{g t^{M} R[[t]]}{\left.t^{n} R[t]\right]}$ has a free resolution of length two

$$
0 \rightarrow t^{n_{1}} R[[t]] \rightarrow g t^{M} R[[t]] \rightarrow \frac{g t^{M} R[[t]]}{t^{n_{1}} R[[t]]} \rightarrow 0
$$

Then $\frac{t^{n_{1}} R[[t]]}{\left.g t^{n_{2}} R[t]\right]}$ is projective since there is the following short exact sequence

$$
0 \rightarrow \frac{t^{n_{1}} R[[t]]}{g t^{n_{2}} R[[t]]} \rightarrow \frac{g t^{M} R[[t]]}{g t^{n_{2}} R[[t]]} \rightarrow \frac{g t^{M} R[[t]]}{t^{n_{1}} R[[t]]} \rightarrow 0
$$

and the middle term is free. Then $\frac{g t^{n_{2}} R[[t]]}{\left.t^{n_{3}} R[t]\right]}$ is projective and finitely generated because of the following short exact sequence

$$
0 \rightarrow \frac{g t^{n_{2}} R[[t]]}{t^{n_{3}} R[[t]]} \rightarrow \frac{t^{n_{1}} R[[t]]}{t^{n_{3}} R[[t]]} \rightarrow \frac{t^{n_{1}} R[[t]]}{g t^{n_{2}} R[[t]]} \rightarrow 0
$$

and the middle term is a free right $R$-module of finite rank.

Therefore

$$
\frac{g t^{M} R[[t]]}{t^{n_{3}} R[[t]]} \cong \frac{g t^{M} R[[t]]}{g t^{n_{2}} R[[t]]} \oplus \frac{g t^{n_{2}} R[[t]]}{t^{n_{3}} R[[t]]}
$$

is a finitely generated projective right $R$-module.

Now we define deg $: G L_{\infty}(R) \rightarrow K_{0}\left(R^{\text {op }}\right)$ as $\left[\frac{R[[t]]}{t^{N} R[[t]]}\right]-\left[\frac{g R[[t]]}{\left.t^{N} R[t]\right]}\right]$, where $N$ is chosen so that $t^{N} R[[t]] \subset g R[[t]]$ and $\frac{g R[[t]]}{\left.t^{N} R[t]\right]}$ is a finitely generated projective right $R$-module. Apparently, this element in $K_{0}\left(R^{\mathrm{op}}\right)$ does not depend on the choice of $N$, and therefore gives a well-defined map deg. It is also easy to check that this is indeed a group homomorphism. 
Next, we prove that deg is surjective. First, observe that any element in $K_{0}\left(R^{\mathrm{op}}\right)$ can be represented as $\left[R^{n}\right]-[P]$ where $R^{n}$ is the free right $R$-module of rank $n$, and $P$ is a finitely generated projective right $R$-module. Now let $Q$ be a right $R$-module such that $P \oplus Q \cong R^{m}$ for some $m$. Now we define $g \in G L_{\infty}(R)$ so that $\operatorname{deg}(g)=\left[R^{n}\right]-[P]$. For $s \geq r$, denote by $R_{r}^{s}$ the free right $R$-module of rank $s-r R t^{r} \oplus R t^{r+1} \oplus \cdots \oplus R t^{s-1}$. Write $R_{n+k m}^{n+(k+1) m}=P_{k} \oplus Q_{k}$, where $P_{k} \cong P$ and $Q_{k} \cong Q$. Then we define an isomorphism $g: R((t)) \rightarrow R((t))$ by sending $R_{k m}^{(k+1) m}$ isomorphically to $P_{k-1} \oplus Q_{k}$, so that $A[[t]] \cong$ $\prod_{k \geq 0} R_{k m}^{(k+1) m}$ is mapped isomorphically to $P_{-1} \oplus \prod_{k \geq 0}\left(Q_{k} \oplus P_{k}\right) \cong P \oplus t^{n} R[[t]]$. It is clear that such an element $g$ is indeed in $G L_{\infty}(R)$, and $\left[\frac{R[t]]}{\left.t^{n} R[t]\right]}\right]-\left[\frac{g R[[t]]}{\left.t^{n} R[t]\right]}\right]=\left[R^{n}\right]-$ $[P]$.

Proposition 4.5. Let $\operatorname{deg}: G L_{\infty}(R) \rightarrow K_{0}\left(R^{\mathrm{op}}\right)$ be the homomorphism constructed in Proposition 4.3. Then we have the following exact sequence of groups:

$$
1 \rightarrow G L_{\mathfrak{f}}(R) \stackrel{i}{\rightarrow} \widetilde{G L}_{\infty}(R) \stackrel{p}{\rightarrow} G L_{\infty}(R) \stackrel{\operatorname{deg}}{\rightarrow} K_{0}\left(R^{\mathrm{op}}\right) \rightarrow 0 .
$$

Proof. It is enough to check the exactness at $G L_{\infty}(R)$. So we have to prove that for $g \in G L_{\infty}(R)$, we have $\operatorname{deg}(g)=0$ if and only if $\pi g=a-f$ for some $a \in G L_{\infty}^{+}(R)$ and $f \in \mathfrak{g l}_{\mathfrak{f}}(R)$.

First, assume that $\pi g=a-f$ with $a \in G L_{\infty}^{+}(R)$ and $f \in \mathfrak{g l}_{\mathfrak{f}}(R)$. Then there exists some $N$, large enough so that $\left.f\right|_{t^{N}} R[[t]]=0$. For $N$ large enough, $g t^{N} R[[t]] \subset R[[t]]$ and therefore $g t^{N} R[[t]]=\pi\left(g t^{N} R[[t]]\right)=(a-f) t^{N} R[[t]]=a t^{N} R[[t]]$. We have

$$
\begin{aligned}
\operatorname{deg}\left(g^{-1}\right) & =\left[\frac{R[[t]]}{\left.t^{N} R[t]\right]}\right]-\left[\frac{g^{-1} R[[t]]}{t^{N} R[[t]]}\right] \\
& =\left[\frac{R[[t]]}{t^{N} R[[t]]}\right]-\left[\frac{R[t]]}{\left.g t^{N} R[t]\right]}\right] \\
& =\left[\frac{R[[t]]}{t^{N} R[[t]]}\right]-\left[\frac{R[[t]]}{\left.a t^{N} R[t]\right]}\right] \\
& =\left[\frac{R[[t]]}{\left.t^{N} R[t]\right]}\right]-\left[\frac{a R[[t]]}{\left.a t^{N} R[t]\right]}\right]=0
\end{aligned}
$$

Conversely, assume that $\operatorname{deg}\left(g^{-1}\right)=\operatorname{deg}(g)=0$, and therefore $\left[\frac{R[[t]]}{\left.t^{N} R[t]\right]}\right]=\left[\frac{g^{-1} R[[t]]}{t^{N} R[[t]]}\right]$ for $N$ large enough. It is well-known that if $[P]=[Q]$ in $K_{0}\left(R^{\mathrm{op}}\right)$, then there exists some $n$ such that $P \oplus R^{n} \cong Q \oplus R^{n}$ as right $R$-modules. Therefore, we could choose $N$ large enough so that

$$
\frac{R[[t]]}{t^{N} R[[t]]} \cong \frac{g^{-1} R[[t]]}{t^{N} R[[t]]} \cong \frac{R[[t]]}{g t^{N} R[[t]]}
$$

We pick such an isomorphism. Observe that since $\frac{R[[t]]}{\left.g t^{N} R[t]\right]}$ is projective, there exists an $R$-submodule $M \subset R[[t]]$, isomorphic to $\frac{R[[t]]}{\left.\left.g t^{N} R[t]\right]\right]}$, such that $R[[t]]=g t^{N} R[[t]] \oplus M$. Then we have an isomorphism $f: \frac{R[[t]]}{\left.t^{N} R[t]\right]} \cong \frac{R[[t]]}{g t^{N} R[[t]]} \cong M$.

Now we define an isomorphism $a \in R[[t]] \rightarrow R[[t]]$ as follows:

$$
R[[t]] \cong t^{N} R[[t]] \oplus R[[t]] / t^{N} R[[t]] \stackrel{g \oplus f}{\rightarrow} g t^{N} R[[t]] \oplus M=R[[t]] .
$$

It is clear from the definition that $a \in G L_{\infty}^{+}(R)$ and $\pi g-\left.a\right|_{t^{N}} R[[t]]=0$, i.e. $\pi g-a \in$ $\mathfrak{g l}_{\mathfrak{f}}(R)$.

Denote $G L_{\infty}(R)^{0}:=\operatorname{ker}\left(G L_{\infty}(R) \rightarrow K_{0}\left(R^{\mathrm{op}}\right)\right)$. Therefore, we have a short exact sequence 


$$
0 \rightarrow G L(R) \rightarrow \widetilde{G L}_{\infty}(R) \rightarrow G L_{\infty}(R)^{0} \rightarrow 1
$$

$G L_{\infty}(\mathbb{C})^{0}$ will be denoted by $G L_{\infty}^{0}$ for simplicity.

We can construct another exact sequence of groups. Apply the functor $G L_{\mathfrak{f}}$ to the short exact sequence of algebras (4.1), we obtain

$$
1 \rightarrow G L_{\mathfrak{f}}\left(\mathfrak{g l}_{\mathfrak{f}}(R)\right) \rightarrow G L_{\mathfrak{f}}\left(\tilde{\mathfrak{g l}}_{\infty}(R)\right) \rightarrow G L_{\mathfrak{f}}\left(\mathfrak{g l}_{\infty}(R)\right)
$$

We have

Proposition 4.6. The above exact sequence extends to

$$
1 \rightarrow G L_{\mathfrak{f}}\left(\mathfrak{g l}_{\mathfrak{f}}(R)\right) \rightarrow G L_{\mathfrak{f}}\left(\tilde{g l}_{\infty}(R)\right) \rightarrow G L_{\mathfrak{f}}\left(\mathfrak{g l}_{\infty}(R)\right) \rightarrow K_{0}\left(R^{\text {op }}\right) \rightarrow 0
$$

Proof. For any $n$, let $G L_{\mathfrak{f}, n}(R)$ be the subgroup of $G L_{\mathfrak{f}}(R)$ consisting of those $a$ for which $\left.a\right|_{t^{n} R[[t]]}=\mathrm{id}$. Then it maps surjective to $G L_{n}(R)$, the group of invertible $n \times n$ matrices with coefficients in $R$, by $p_{n}$, where

$$
p_{n}\left(a=\left(a_{i j}\right)_{i, j \geq 0}\right)=\left(a_{i j}\right)_{0 \leq i, j \leq n-1} .
$$

Denote by $i_{n, n+1}$ the inclusion of $G L_{\mathfrak{f}, n}(R)$ to $G L_{\mathfrak{f}, n+1}(R)$.

We have isomorphisms $\varphi_{n}: G L_{n}\left(\mathfrak{g l}_{\mathfrak{f}}(R)\right) \cong G L_{\mathfrak{f}}(R), G L_{n}\left(\widetilde{\mathfrak{g l}}_{\infty}(R)\right) \cong \widetilde{G L}_{\infty}(R)$, and $G L_{n}\left(\mathfrak{g l}_{\infty}(R)\right) \cong G L_{\infty}(R)$ making the following diagram commutative

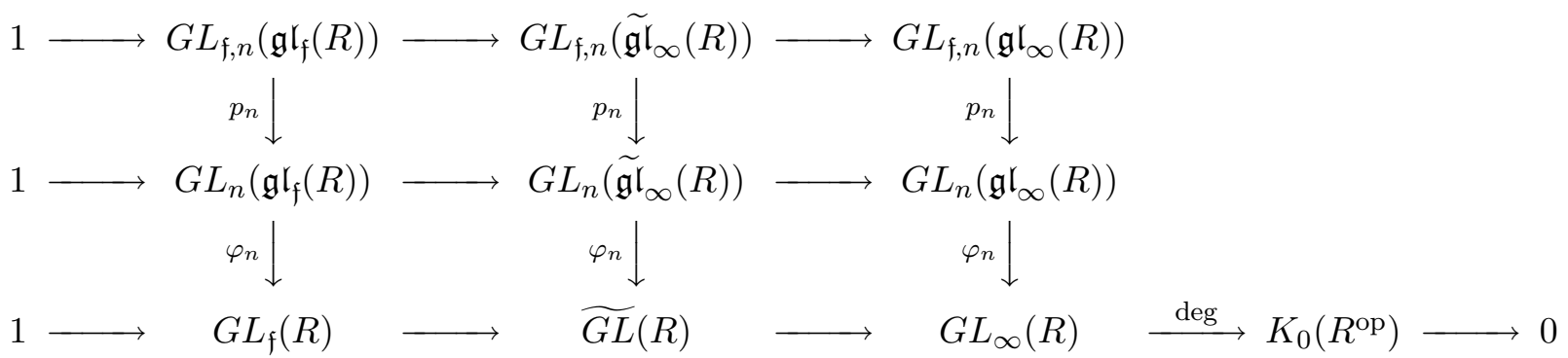

Using this diagram, it is easy to see that the sequence

$$
G L_{\mathfrak{f}, n}\left(\tilde{\mathfrak{g l}}_{\infty}(R)\right) \rightarrow G L_{\mathfrak{f}, n}\left(\mathfrak{g l}_{\infty}(R)\right) \stackrel{\varphi_{n} \circ p_{n}}{\longrightarrow} K_{0}\left(R^{\mathrm{op}}\right)
$$

is exact at $G L_{\mathfrak{f}, n}\left(\mathfrak{g l}_{\infty}(R)\right)$. Furthermore, it is easy to check that, although $\varphi_{n} \circ p_{n} \neq$ $\varphi_{n+1} \circ p_{n+1} \circ i_{n, n+1}$, we have $\operatorname{deg} \circ \varphi_{n} \circ p_{n}=\operatorname{deg} \circ\left(\varphi_{n+1} \circ p_{n+1} \circ i_{n, n+1}\right)$. This proves the proposition.

Remark 4.7. Observe that we have an isomorphism

$$
G L_{\mathfrak{f}}(R) \cong G L\left(\mathfrak{g l}_{\mathfrak{f}}(R)\right)=\underset{\vec{n}}{\lim _{n}} G L_{n}\left(\mathfrak{g l}_{\mathfrak{f}}(R)\right)
$$

(not compatible with any $\varphi_{n}$ above).

Let $G L_{\mathfrak{f}}\left(\mathfrak{g l}_{\infty}(R)\right)^{0}$ be the kernel of $G L_{\mathfrak{f}}\left(\mathfrak{g l}_{\infty}(R)\right) \rightarrow K_{0}\left(R^{\mathrm{op}}\right)$. Thus, we have another exact sequence,

$$
1 \rightarrow G L_{\mathfrak{f}}\left(\mathfrak{g l}_{\mathfrak{f}}(R)\right) \rightarrow G L_{\mathfrak{f}}\left(\tilde{g l}_{\infty}(R)\right) \rightarrow G L_{\mathfrak{f}}\left(\mathfrak{g l}_{\infty}(R)\right)^{0} \rightarrow 1
$$


4.2.3. $H^{2}$ and central extensions. Let $R=\mathbb{C}$. Then we have the exact sequence (1.3),

$$
1 \rightarrow G L_{\mathfrak{f}} \rightarrow \widetilde{G L}_{\infty} \rightarrow G L_{\infty}^{0} \rightarrow 1 .
$$

Pushing out this sequence by det, we obtain a central extension of $G L_{\infty}^{0}$.

$$
1 \rightarrow \mathbb{C}^{\times} \rightarrow \widehat{G L}_{\infty}^{0} \rightarrow G L_{\infty}^{0} \rightarrow 1
$$

which we constructed in 91 , The cohomology class $\left[C_{2}^{0}\right] \in H^{2}\left(G L_{\infty}^{0}, \mathbb{C}^{\times}\right)$is obtained by the transgression of $\left[\right.$ det] $\in H^{1}\left(G L_{\mathfrak{f}}, \mathbb{C}^{\times}\right)$. We claim that it is non-trivial. This is because in the Lyndon-Hochschild-Serre spectral sequence associated to (1.3), $\left[C_{2}^{0}\right] \in$ $E_{2}^{2,0}$ is obtained by transgression of [det] $\in E_{2}^{0,1}$. If $\left[C_{2}^{0}\right]=0$, then [det] would survive to the $E_{\infty}$ term, so that $H^{1}\left(\widetilde{G L}_{\infty}, \mathbb{C}^{\times}\right) \neq 0$, which contradicts Proposition 4.2 .

It was proved in Proposition 1.3 that such central extension can be extended to a unique central extension $\widehat{G L}_{\infty}$ of $G L_{\infty}$.

We have a similar story for $G L_{\mathfrak{f}, \infty}$. We only sketch it, since it is almost a word-forword repetition of the story of $G L_{\infty}$. Let $R=\mathbb{C}$ in (4.3), we obtain

$$
1 \rightarrow G L_{\mathfrak{f}}\left(\mathfrak{g l}_{\mathfrak{f}}\right) \rightarrow G L_{\mathfrak{f}}\left(\tilde{\mathfrak{g l}}_{\infty}\right) \rightarrow G L_{\mathfrak{f}, \infty}^{0} \rightarrow 1 \text {. }
$$

We claim that there is a well-defined group homomorphism det $: G L_{\mathfrak{f}}\left(\mathfrak{g l}_{\mathfrak{f}}\right) \rightarrow \mathbb{C}^{\times}$. Indeed, for any $n$, we have the homomorphisms

$$
G L_{\mathfrak{f}, n}\left(\mathfrak{g l}_{\mathfrak{f}}\right) \rightarrow G L_{n}\left(\mathfrak{g l}_{\mathfrak{f}}\right) \cong G L_{\mathfrak{f}} \stackrel{\operatorname{det}}{\rightarrow} \mathbb{C}^{\times},
$$

which are compatible with the embeddings $i_{n, n+1}$. This gives det $: G L_{\mathfrak{f}}\left(\mathfrak{g l}_{\mathfrak{f}}\right) \rightarrow \mathbb{C}^{\times}$. Now pushing-out the above sequence by det, we obtain

$$
1 \rightarrow \mathbb{C}^{\times} \rightarrow \widehat{G L}_{\mathfrak{f}, \infty}^{0} \rightarrow G L_{\mathfrak{f}, \infty}^{0} \rightarrow 1
$$

One can similarly prove the following:

Proposition 4.8. (i) The extension (4.4) is non-trivial. The cohomology class $\left[D_{2}^{0}\right] \in$ $H^{2}\left(G L_{\mathfrak{f}, \infty}^{0}, \mathbb{C}^{\times}\right)$is the transgression of $[\operatorname{det}] \in H^{1}\left(G L_{\mathfrak{f}}\left(\mathfrak{g l}_{\mathfrak{f}}\right), \mathbb{C}^{\times}\right)$.

(ii) There is a unique (up to an isomorphism) central extension

$$
1 \rightarrow \mathbb{C}^{\times} \rightarrow \widehat{G L}_{\mathfrak{f}, \infty} \rightarrow G L_{\mathfrak{f}, \infty} \rightarrow 1
$$

whose restriction to $G L_{\mathfrak{f}, \infty}^{0}$ is (4.4). The cohomology class corresponding to this central extension is denoted by $\left[D_{2}\right]$.

Proof. The only fact we use is that $H^{1}\left(G L_{\mathfrak{f}}\left(\widetilde{\mathfrak{g l}}_{\infty}\right), \mathbb{C}^{\times}\right)=0$. This is because $\widetilde{G L}_{\infty} \subset$ $G L_{\mathfrak{f}}\left(\widetilde{\mathfrak{g l}}_{\infty}\right)$ and $H^{1}\left(\widetilde{G L}_{\infty}, \mathbb{C}^{\times}\right)=0$. Then (i) follows from the same argument as the proof of the non-triviality of $\left[C_{2}^{0}\right]$, and (ii) follows from the proof of Proposition 1.3.

As before, the automorphism of (4.5) is $H^{1}\left(G L_{\mathfrak{f}, \infty}, \mathbb{C}^{\times}\right)=\mathbb{C}^{\times}$.

4.2.4. $\widehat{G L}_{\mathfrak{f}, \infty}$ as the central extension of $G L_{\mathfrak{f}, \infty}$ by determinant lines. We can also regard $\widehat{G L}_{\mathfrak{f}, \infty}$ as the central extension of $G L_{\mathfrak{f}, \infty}$ by determinant lines. Recall that $\mathcal{O}_{\mathbb{K}}=\mathbb{C}((t))[[s]]$. This is a lattice of $\mathbb{K}$ (see Definition [3.3). Let $L, L^{\prime}$ be two secondary lattices of $\mathcal{O}_{\mathbb{K}}$ (see Definition 3.6) We assume that there exists some $n$ such that $L \cap s^{n} \mathcal{O}_{\mathbb{K}}=L^{\prime} \cap s^{n} \mathcal{O}_{\mathbb{K}}$. Observe that although $L$ and $L^{\prime}$ may not be commensurable 
with each other (an example being given below), we can still define the determinant line $\operatorname{det}\left(L \mid L^{\prime}\right)$. This is because for any $m \geq n$, there is a canonical isomorphism

$$
\operatorname{det}\left(\frac{L}{L \cap s^{m} \mathcal{O}_{\mathbb{K}}} \mid \frac{L^{\prime}}{L^{\prime} \cap s^{m} \mathcal{O}_{\mathbb{K}}}\right) \stackrel{\cong}{\operatorname{det}}\left(\frac{L}{L \cap s^{n} \mathcal{O}_{\mathbb{K}}} \mid \frac{L^{\prime}}{L^{\prime} \cap s^{n} \mathcal{O}_{\mathbb{K}}}\right) .
$$

Then we can define

$$
\operatorname{det}\left(L \mid L^{\prime}\right):=\lim _{\overleftarrow{m}} \operatorname{det}\left(\frac{L}{L \cap s^{m} \mathcal{O}_{\mathbb{K}}} \mid \frac{L^{\prime}}{L^{\prime} \cap s^{m} \mathcal{O}_{\mathbb{K}}}\right) .
$$

It is clear that if $L$ and $L^{\prime}$ are commensurable, the new definition coincides with the old one. This motivates us to define

Definition 4.9. Let $\mathbb{L}$ be a lattice in $\mathbb{K}$ and $L, L^{\prime} \subset \mathbb{L}$ are two secondary lattices. We will call $L, L^{\prime}$ pseudo commensurable with each other if there exists some lattice $\mathbb{L}^{\prime} \subset \mathbb{L}$ such that $L \cap \mathbb{L}^{\prime}=L^{\prime} \cap \mathbb{L}^{\prime}$. In this case, we $\operatorname{define} \operatorname{det}\left(L \mid L^{\prime}\right)$ by formula (4.7).

Let us emphasize that the important properties of $\operatorname{det}\left(L \mid L^{\prime}\right)$ are those stated in Remark 1.5. That is, for $L, L^{\prime}, L^{\prime \prime}$ pseudo commensurable with each other, there is a canonical isomorphism

$$
\gamma_{L, L^{\prime}, L^{\prime \prime}}: \operatorname{det}\left(L \mid L^{\prime}\right) \otimes \operatorname{det}\left(L^{\prime} \mid L^{\prime \prime}\right) \cong \operatorname{det}\left(L \mid L^{\prime \prime}\right)
$$

such that for any $L, L^{\prime}, L^{\prime \prime}, L^{\prime \prime \prime}, \gamma_{L, L^{\prime}, L^{\prime \prime \prime}} \gamma_{L^{\prime}, L^{\prime \prime}, L^{\prime \prime \prime}}=\gamma_{L, L^{\prime \prime}, L^{\prime \prime \prime}} \gamma_{L, L^{\prime}, L^{\prime \prime}}$.

Now let $G L_{\infty}^{+}\left(\mathfrak{g l}_{\infty}\right)$ acts on $\mathcal{O}_{\mathbb{K}}$ via the following formula. If we represent an element in $G L_{\infty}^{+}\left(\mathfrak{g l}_{\infty}\right)$ as $a=\left(a_{i j, m n}\right)_{i, j \geq 0, m, n \in \mathbb{Z}}$, then

$$
a\left(t^{n} s^{j}\right)=\sum_{m \in \mathbb{Z}, n \geq 0} a_{i j, m n}\left(t^{m} s^{i}\right) .
$$

The following lemmas are easy to check.

Lemma 4.10. For any $a \in G L_{\infty}^{+}\left(\mathfrak{g l}_{\infty}\right)$, and a secondary lattice $L \subset \mathcal{O}_{\mathbb{K}}$, aL is a secondary lattice. In fact, $G L_{\infty}^{+}\left(\mathfrak{g l}_{\infty}\right)$ acts transitively on the set of secondary lattices in $\mathcal{O}_{\mathbb{K}}$. Furthermore, if $a \in G L_{\mathfrak{f}, \infty} \subset G L_{\infty}^{+}\left(\mathfrak{g l}_{\infty}\right)$, then aL is pseudo commensurable with $L$, i.e., $\exists n$ such that $L \cap s^{n} \mathcal{O}_{\mathbb{K}}=a L \cap s^{n} \mathcal{O}_{\mathbb{K}}$.

Lemma 4.11. If $L, L^{\prime}$ are two secondary lattices in $\mathcal{O}_{\mathbb{K}}$, pseudo commensurable with each other, then for $a \in G L_{\infty}^{+}\left(\mathfrak{g l}_{\infty}\right), a L, a L^{\prime}$ are pseudo commensurable, and a induces an isomorphism $\operatorname{det}\left(L \mid L^{\prime}\right) \stackrel{\cong}{\cong} \operatorname{det}\left(a L \mid a L^{\prime}\right)$.

Example 4.12. Let $L_{0}=\mathbb{C}[[t]][[s]]$. Observe that if we let $a \in G L_{\mathfrak{f}, \infty}$ which is defined by

$$
a t^{i}=\sum_{j \geq 0} s^{j} t^{i-j}, \quad a\left(s^{i} t^{j}\right)=s^{i} t^{j}
$$

then $a L_{0} \cap L_{0}=s \mathbb{C}[[t]][[s]]$. Therefore, $a L_{0}$ is not commensurable with $L_{0}$.

Recall the definition of $G L_{\mathfrak{f}, n}\left(\mathfrak{g l}_{\infty}\right)$, which will be denoted by $G L_{n, \infty}$ in what follows for simplicity. This is a subgroup of $G L_{\mathfrak{f}, \infty}$ consisting of $a: \mathfrak{g l}_{\infty}[[s]] \rightarrow \mathfrak{g l}_{\infty}[[s]]$ such that $\left.a\right|_{s^{n} \mathfrak{g l}_{\infty}[[s]]}=\mathrm{id}$. We denote the restriction of the central extensions $\widehat{G L}_{\mathfrak{f}, \infty}$ of $G L_{\mathfrak{f}, \infty}$ to $G L_{n, \infty}$ by $\widehat{G L}_{n, \infty}$. Let $L$ be a secondary lattice of $\mathcal{O}_{\mathbb{K}}$. As shown in Proposition 1.6, 
the central extension $\widehat{G L}_{n, \infty}$ can be interpreted as the group consisting of $(a, e)$, where $a \in G L_{n, \infty}$ and

$$
e \in \operatorname{det}\left(\frac{a L}{a L \cap s^{n} \mathcal{O}_{\mathbb{K}}} \mid \frac{L}{L \cap s^{n} \mathcal{O}_{\mathbb{K}}}\right)^{\times}=\operatorname{det}\left(\frac{a L}{L \cap s^{n} \mathcal{O}_{\mathbb{K}}} \mid \frac{L}{L \cap s^{n} \mathcal{O}_{\mathbb{K}}}\right)^{\times} .
$$

We thus obtain

Proposition 4.13. The central extensions $\widehat{G L}_{\mathfrak{f}, \infty}$ of $G L_{\mathfrak{f}, \infty}$ can be interpreted as the group consisting of $(a, e)$, where $a \in G L_{\mathfrak{f}, \infty}$ and $e \in \operatorname{det}(a L \mid L)^{\times}$for any secondary lattice $L \subset \mathcal{O}_{\mathbb{K}}$.

Observe that $G L_{\infty}^{+}\left(\mathfrak{g l}_{\infty}\right)$ acts on $G L_{\mathfrak{f}, \infty}$ by conjugation $\rho$, i.e., $\rho_{g}(h)=g h g^{-1}$ for $g \in G L_{\infty}^{+}\left(\mathfrak{g l}_{\infty}\right), h \in G L_{\mathfrak{f}, \infty}$.

Corollary 4.14. Let $g \in G L_{\infty}^{+}\left(\mathfrak{g l}_{\infty}\right)$. Then the central extension $\mathbb{C}^{\times} \rightarrow \widehat{G L}_{\mathfrak{f}, \infty}^{g} \rightarrow$ $G L_{\mathfrak{f}, \infty}$ obtained by pullback of $\mathbb{C}^{\times} \rightarrow \widehat{G L}_{\mathfrak{f}, \infty} \rightarrow G L_{\mathfrak{f}, \infty}$ along $\rho_{g}: G L_{\mathfrak{f}, \infty} \rightarrow G L_{\mathfrak{f}, \infty}$ is isomorphic to $\widehat{G L}_{\mathfrak{f}, \infty}$.

Proof. If we present $\widehat{G L}_{\mathfrak{f}, \infty}$ as the group consisting of elements $(a, e)$, where $a \in G L_{\mathfrak{f}, \infty}$ and $e \in \operatorname{det}(a L \mid L)^{\times}$for any secondary lattice $L \subset \mathcal{O}_{\mathbb{K}}$, then $\widehat{G L}_{\mathfrak{f}, \infty}^{g}$ will have similar presentation where $L$ is replaced by $g^{-1} L$.

4.2.5. An extension of $G L_{\infty, \infty}$ by $G L_{\mathfrak{f}, \infty}$. We first claim the following:

Lemma 4.15. $K_{0}\left(\mathfrak{g l}_{\infty}^{\mathrm{op}}\right)=0$ so that $G L_{\infty, \infty}^{0}=G L_{\infty, \infty}$.

Proof. We define a functor from the category of finitely generated projective $\mathfrak{g l}_{\infty}$ modules to the category of Tate vector spaces by $P \mapsto P \otimes_{\mathfrak{g l}_{\infty}} K$. (We recall hat $K=\mathbb{C}((t))$ and $\left.\mathfrak{g l}_{\infty}=\operatorname{End} K.\right)$ Fixing a finite set $\left\{p_{i}\right\}$ of generators of $P$ as a right $\mathfrak{g l}_{\infty}$-module, a basis of open neighborhoods of $0 \in P \otimes_{\mathfrak{g l}_{\infty}} K$ is given by $\sum_{i} p_{i} \otimes U_{i}$, where $U_{i}$ vary in the set of open subspaces of $K$. It is easy to see that the topology on $P \otimes_{\mathfrak{g l}_{\infty}} K$ is independent of the choice of $\left\{p_{i}\right\}$. Observe that $\operatorname{Hom}\left(K, P \otimes_{\mathfrak{g l}_{\infty}} K\right)$ is a right $\operatorname{Hom}(K, K)=\mathfrak{g l}_{\infty}$-module. We first show that $\operatorname{Hom}\left(K, P \otimes_{\mathfrak{g l}_{\infty}} K\right) \cong P$.

Indeed, using an isomorphism $K \oplus K \cong K$ of Tate vector spaces, we obtain $\mathfrak{g l}_{\infty} \oplus$ $\mathfrak{g l}_{\infty}=\operatorname{Hom}(K, K \oplus K) \cong \operatorname{Hom}(K, K)=\mathfrak{g l}_{\infty}$. Therefore, any finitely generated projective right $\mathfrak{g l}_{\infty}$ is a direct summand of $\mathfrak{g l}_{\infty}$, in particular a cyclic right $\mathfrak{g l}_{\infty}$-module. Let $p \in \mathfrak{g l}_{\infty}$ be a generator of $P$ (so $P=p \mathfrak{g l}_{\infty} \subset \mathfrak{g l}_{\infty}$ ). We thus obtain that $\operatorname{Hom}\left(K, P \otimes_{\mathfrak{g l}_{\infty}} K\right)$ is a direct summand of $\operatorname{Hom}\left(K, \mathfrak{g l}_{\infty} \otimes_{\mathfrak{g l}_{\infty}} K\right) \cong \mathfrak{g l}_{\infty}$. Observe that under the last isomorphism, the map $\varphi \in \operatorname{Hom}\left(K, \mathfrak{g l}_{\infty} \otimes_{\mathfrak{g l}_{\infty}} K\right)$ defined by $\varphi(v)=p \otimes v$ goes to $p$. This proves that $\operatorname{Hom}\left(K, P \otimes_{\mathfrak{g l}_{\infty}} K\right) \cong P$.

Now, using the fact that for any Tate subspace $W \subset K$, we have $W \oplus K \cong K$ as Tate vector spaces, we obtain that

$$
P \oplus \mathfrak{g l}_{\infty} \cong \operatorname{Hom}\left(K, P \otimes_{\mathfrak{g l}_{\infty}} K \oplus K\right) \cong \operatorname{Hom}(K, K)=\mathfrak{g l}_{\infty} .
$$

This implies that in $K_{0}\left(\mathfrak{g} \mathfrak{l}_{\infty}^{\mathrm{op}}\right),[P]=0$.

Remark 4.16. (i) For any $R$, we thus obtain a functor from the category $\mathcal{P}_{R}$ of finitely generated projective $\mathfrak{g l}_{\infty}(R)$-modules to the category $\mathcal{T}_{R}$ of Tate $R$-modules (see [Dr], Definition 3.2.1, for the definition of Tate $R$-modules). At the level of Grothendieck groups, this gives an isomorphism $K_{0}\left(\mathfrak{g l}_{\infty}(R)\right)=K_{0}\left(\mathcal{P}_{R}\right) \cong K_{0}\left(\mathcal{T}_{R}\right) \cong K_{-1}(R)$. (See [Dr], Theorem 3.6.) 
(ii) A variation of the main result in [W] generalizes the result in Remark (i). Namely, the functor $\mathfrak{g l}_{\infty}$ shifts the algebraic $K$-theory of a ring by degree one, i.e., $K_{*}\left(\mathfrak{g l}_{\infty}(R)\right)=$ $K_{*-1}(R)$.

(iii) There is also an additive analogue of Remark (ii), see [FT2], Proposition 4.1.5.

Now let $R=\mathfrak{g l}_{\infty}$ in (4.2). By the above lemma, we have an exact sequence of groups

$$
1 \rightarrow G L_{\mathfrak{f}, \infty} \rightarrow \widetilde{G L}_{\infty}\left(\mathfrak{g l}_{\infty}\right) \rightarrow G L_{\infty, \infty} \rightarrow 1 .
$$

Since $H^{\bullet}\left(\widetilde{G L}_{\infty}\left(\mathfrak{g l}_{\infty}\right)\right) \cong \mathbb{Z}$ and $H^{1}\left(G L_{\mathfrak{f}, \infty}, \mathbb{Z}\right) \cong \mathbb{Z}$, by the spectral sequence associated to (4.9), we obtain that $H^{2}\left(G L_{\infty, \infty}, \mathbb{Z}\right) \cong \mathbb{Z}$. The $\mathbb{Z}$-central extension

$$
1 \rightarrow \mathbb{Z} \rightarrow \widehat{G L}_{\infty, \infty} \rightarrow G L_{\infty, \infty} \rightarrow 1
$$

of $G L_{\infty, \infty}$ corresponding to the generator of $H^{2}\left(G L_{\infty, \infty}, \mathbb{Z}\right)$ can be obtained as the push-out of (4.5) by $\operatorname{deg}: G L_{\mathfrak{f}, \infty} \rightarrow \mathbb{Z}$. This is the universal $\mathbb{Z}$-central extension of $G L_{\infty, \infty}$. Observe that $\widehat{G L}_{\infty, \infty}$ fits into the following exact sequence of groups

$$
1 \rightarrow G L_{\mathfrak{f}, \infty}^{0} \rightarrow \widetilde{G L}_{\infty}\left(\mathfrak{g l}_{\infty}\right) \rightarrow \widehat{G L}_{\infty, \infty} \rightarrow 1
$$

Remark 4.17. We remark that the group $\widehat{G L}_{\infty, \infty}$ plays a central role in Osipov's work of reciprocity laws on algebraic surfaces (see Osi1]).

In the next section, we will obtain a nontrivial cohomology class $\left[E_{3}\right] \in H^{3}\left(G L_{\infty, \infty}\right)$ (see Theorem 5.7) from the sequence (4.9).

\section{Cohomology Classes of gerbal representations of $G L_{\infty, \infty}$}

In this section we compute the third cohomology class realized in the gerbal representation of $G L_{\infty, \infty}$ on the category $\mathcal{C}_{\mathcal{O}_{\mathbb{K}}}^{\text {ss }}$ constructed in Theorem 3.11, The main result is Theorem 5.9. In order to prove this result we realize this class as the cohomology class arising from another gerbal representation of $G L_{\infty, \infty}$. We then use some general results presented below on what we call "gerbal pairs of groups".

5.1. Gerbal pairs of groups. In this section we develop a formalism which allows us to calculate the cohomology classes corresponding to gerbal representations in a certain situation. This is based on what we call gerbal pairs of groups. In this section, we will first recall one construction of the Lyndon-Hochschild-Serre spectral sequences. Then we will develop the general formalism of gerbal pairs of groups. Finally, we will show that there is an action of $\widetilde{G L}_{\infty}\left(\mathfrak{g l}_{\infty}\right)$ on $\widehat{G L}_{\mathfrak{f}, \infty}$, such that the groups $\left(\widetilde{G L}_{\infty}\left(\mathfrak{g l}_{\infty}\right), \widehat{G L}_{\mathfrak{f}, \infty}\right)$ equipped with the short exact sequences (4.5) and (4.9) form a gerbal pair in the sense of Definition 5.1.

\subsubsection{The spectral sequence of a group extension. Recall that if}

$$
1 \rightarrow H \rightarrow G \rightarrow K \rightarrow 1
$$

is an extension of groups, then for a $G$-module $M$ the Lyndon-Hochschild-Serre spectral sequence has the following second term:

$$
E_{2}^{p, q}=H^{p}\left(K, H^{q}(H, M)\right) \Rightarrow H^{p+q}(G, M) .
$$


We will recall one construction of this spectral sequence given in Chapter II of [HS]. It uses an appropriate filtration on the complex of normalized cochains $C^{\bullet}(G, M)$. Recall that for $M$ a $G$-module, the cohomology $H^{\bullet}(G, M)$ can be calculated by the complex of "normalized" cochains $C^{\bullet}(G, M)$, where $C^{n}(G, M)$ is the group of maps $f: G^{n} \rightarrow M$ such that $f\left(g_{1}, \ldots, g_{n}\right)=0$ whenever one of the $g_{i}$ is the identity. The coboundary map $\delta_{G}: C^{n}(G, M) \rightarrow C^{n+1}(G, M)$ is given by

$$
\begin{aligned}
\left(\delta_{G} f\right)\left(g_{1}, \ldots, g_{n+1}\right)= & g_{1} f\left(g_{2}, \ldots, g_{n+1}\right) \\
& +\sum(-1)^{i} f\left(g_{1}, \ldots, g_{i} g_{i+1}, \ldots, g_{n+1}\right) . \\
& +(-1)^{n+1} f\left(g_{1}, \ldots, g_{n}\right)
\end{aligned}
$$

Now let $H$ be a normal subgroup of $G$ with $K=G / H$ the quotient. Then we have a filtration on $F^{\bullet} C^{\bullet}(G, M)$ defined by the formula

$$
F^{p} C^{n}(G, M)= \begin{cases}\operatorname{Maps}\left(G^{n-p} \times K^{p}, M\right) \cap C^{n}(G, M) & p \leq n \\ 0 & p>n\end{cases}
$$

The filtration gives rise to a spectral sequence which converges to $H^{\bullet}(G, M)$. Let us recall the $E_{1}$ and $E_{2}$ terms. There is a natural map

$$
\text { Res : } F^{p} C^{n}(G, M) \rightarrow C^{p}\left(K, C^{n-p}(H, M)\right)
$$

obtained by restriction. A key observation of Hochschild and Serre is that this map induces an isomorphism $\left(E_{1}^{*, q}, d_{1}\right) \cong\left(C^{\bullet}\left(K, H^{q}(H, M)\right), \delta_{K}\right)$. This gives the desired spectral sequence with $E_{2}^{p, q} \cong H^{p}\left(K, H^{q}(H, M)\right)$.

In particular, we have the following commutative diagram:

$$
\begin{aligned}
& \begin{array}{ccc}
E_{1}^{0,2}=\frac{\left\{a \in C^{2}, \delta_{G} a \in F^{1} C^{3}\right\}}{\delta_{G}\left(C^{1}\right)+F^{1} C^{2}} & \stackrel{\cong}{\text { Res }} & H^{2}(H, M) \\
\bigcup & & \bigcup
\end{array} \\
& E_{2}^{0,2}=\frac{\left\{a \in C^{2}, \delta_{G} a \in F^{2} C^{3}\right\}}{\delta_{G}\left(C^{1}\right)+\left\{a \in F^{1} C^{2}, \delta_{G} a \in F^{2} C^{3}\right\}} \stackrel{\text { Res }}{\cong} \quad H^{2}(H, M)^{K} \\
& \bigcup
\end{aligned}
$$



5.1.2. The definition and a criterion for gerbal pairs of groups. Let

$$
1 \rightarrow \mathbb{C}^{\times} \rightarrow \widehat{H} \stackrel{\pi}{\rightarrow} H \rightarrow 1
$$

be a central extension of group $H$ by $\mathbb{C}^{\times}$, which corresponds the cohomology class $a \in H^{2}\left(H, \mathbb{C}^{\times}\right)$. Then the conjugation of $\widehat{H}$ on itself descends to an action of $H$ on $\widehat{H}$, which is denoted by $c$. That is, for any $h \in H, \widehat{h} \in \widehat{H}$, choose any lifting $\tilde{h} \in \widehat{H}$ of $h$, then

$$
c_{h}(\widehat{h})=\tilde{h} \widehat{h} \tilde{h}^{-1}
$$

Now assume that we have an extension of groups

$$
1 \rightarrow H \stackrel{i}{\rightarrow} G \rightarrow K \rightarrow 1
$$

Since $H$ is a normal subgroup of $G, G$ acts on $H$ by conjugation, which is denoted by $\rho, \rho_{g}(h)=g h g^{-1}$. 
Definition 5.1. Suppose that we can lift $\rho$ to an action $\tilde{\rho}$ of $G$ on $\widehat{H}$, i.e. $\pi\left(\tilde{\rho}_{g}(\widehat{h})\right)=$ $\rho_{g}(\pi(\widehat{h}))$, so that $\tilde{\rho}_{h}=c_{h}$ for $h \in H$ and $\left.\tilde{\rho}_{g}\right|_{\mathbb{C}^{\times}}=$id. Then we will call the pair $(G, \widehat{H})$ of groups equipped with the short exact sequences (5.1) and (5.2), and the action $\tilde{\rho}$, a gerbal pair.

Note that if $(G, \widehat{H})$ is a gerbal pair of groups, then $\widehat{H} \rightarrow G$ form what is called a "crossed module of groups" (see [Br] $)$.

The following proposition gives the necessary and sufficient conditions for $(G, \widehat{H})$ to be a gerbal pair.

Proposition 5.2. The lifting of Definition 5.1 exists if and only if the class a $\in$ $H^{2}\left(H, \mathbb{C}^{\times}\right)$is transgressive, that is, $a \in H^{2}\left(H, \mathbb{C}^{\times}\right)^{K}$ and $d_{2}(a)=0$, where $d_{2}$ : $H^{2}\left(H, \mathbb{C}^{\times}\right) \rightarrow H^{2}\left(K, H^{1}\left(H, \mathbb{C}^{\times}\right)\right)$is the differential in the $E_{2}$ term of the LyndonHochschild-Serre spectral sequence. If such a lifting exists, then all liftings form a torsor under $Z^{1}\left(K, H^{1}\left(H, \mathbb{C}^{\times}\right)\right)$.

Proof. For any $g \in G$, it acts on $H$ by conjugation and therefore, acts on the cohomology $H^{\bullet}\left(H, \mathbb{C}^{\times}\right)$. Denote this (right) action also by $\rho_{g}$. More explicitly, the class $\rho_{g}(a)$ is obtained by pullback $\mathbb{C}^{\times} \rightarrow \widehat{H} \rightarrow H$ by $\rho_{g}: H \rightarrow H$. Denote the new central extension by $\mathbb{C}^{\times} \rightarrow \widehat{H}_{g} \rightarrow H$. Then $\rho_{g}$ could lift to $\widetilde{\rho_{g}}$, which is the identity map on the central $\mathbb{C}^{\times} \rightarrow \widehat{H}$ if and only if there is an isomorphism $\widehat{H} \rightarrow \widehat{H}_{g}$, which induces the identity maps on the central $\mathbb{C}^{\times}$and the quotient $H$, i.e. $\rho_{g}(a)=a$. Furthermore, all such liftings are bijective to the isomorphisms from $\widehat{H} \rightarrow \widehat{H}_{g}$, and therefore is a torsor under $H^{1}\left(H, \mathbb{C}^{\times}\right)$. Therefore, a lifting $\tilde{\rho}$ exists only if $a$ is a class invariant under $G$. It is a well-known fact that $H$ acts on its cohomology trivially. Therefore, such a lifting exists only if $a \in H^{2}\left(H, \mathbb{C}^{\times}\right)^{K}$.

We assume that $a \in H^{2}\left(H, \mathbb{C}^{\times}\right)^{K}$. We will identify a lifting $\widetilde{\rho_{g}}: \widehat{H} \rightarrow \widehat{H}$ with an isomorphism $\widetilde{\rho_{g}}: \widehat{H} \rightarrow \widehat{H}_{g}$. For every $g \in G$, we choose a lifting of $\widetilde{\rho_{g}}$ of $\rho_{g}$ such that

$$
\widetilde{\rho_{1}}=1, \quad \widetilde{\rho_{g h}}=\widetilde{\rho_{g}} c_{h} \text { for } g \in G, h \in H
$$

For any two $g, g^{\prime} \in G$, both $\widetilde{\rho_{g g^{\prime}}}$ and $\widetilde{\rho_{g}} \widetilde{\rho_{g^{\prime}}}$ are liftings of $\rho_{g g^{\prime}}$. Therefore, there is a unique $\bar{b}\left(g, g^{\prime}\right) \in H^{1}\left(H, \mathbb{C}^{\times}\right)$sending the second lifting to the first lifting. That is, the unique automorphism $\bar{b}\left(g, g^{\prime}\right): \widehat{H} \rightarrow \widehat{H}$ making the following diagram commute.



This way one defines $\bar{b} \in C^{2}\left(G, H^{1}\left(H, \mathbb{C}^{\times}\right)\right)$. We will check that

(i) $\bar{b}\left(h g, g^{\prime}\right)=\bar{b}\left(g, g^{\prime} h\right)=\bar{b}\left(g, g^{\prime}\right)$. Therefore, $\bar{b}$ is in fact in $C^{2}\left(K, H^{1}\left(H, \mathbb{C}^{\times}\right)\right)$.

(ii) Define $b\left(g, g^{\prime}\right)=\bar{b}\left(g^{\prime-1}, g^{-1}\right)$. Then $b$ is a cocycle. Choosing different liftings $\widetilde{\rho_{g}}$ satisfying $(\dagger), b$ differs by a coboundary. Therefore, $[b]$ is a well defined cohomology class in $H^{2}\left(K, H^{1}\left(H, \mathbb{C}^{\times}\right)\right)$.

(iii) If $[b]=0$ in $H^{2}\left(K, H^{1}\left(H, \mathbb{C}^{\times}\right)\right)$, we can choose a compatible family of liftings $\widetilde{\rho_{g}}$ such that $\tilde{\rho}_{g}=\tilde{\rho}_{g}$ is a lifting of $\rho$.

(iv) All the liftings form a torsor under $Z^{1}\left(K, H^{1}\left(H, \mathbb{C}^{\times}\right)\right)$.

(v) $[b]=d_{2}(a)$. 
We begin with the proof of (i). $\bar{b}\left(h g, g^{\prime}\right)=\bar{b}\left(g, g^{\prime}\right)$ comes from the following commutative diagram:



Similarly, we obtain the other identity.

Next, we prove (ii). Observe that we have the following commutative diagram



Therefore, $\bar{b}\left(g^{\prime}, g^{\prime \prime}\right) \bar{b}\left(g g^{\prime}, g^{\prime \prime}\right)^{-1} \bar{b}\left(g, g^{\prime} g^{\prime \prime}\right)\left(\rho_{g^{\prime \prime}} \bar{b}\left(g, g^{\prime}\right)\right)^{-1}=1$. It is readily to check that $b\left(g, g^{\prime}\right)=\bar{b}\left(g^{\prime-1}, g^{-1}\right)$ is a cocycle. If we choose different liftings ${\widetilde{\rho_{g}}}^{\prime}$ satisfying $(\dagger)$, then ${\widetilde{\rho_{g}}}^{\prime}=\widetilde{\rho_{g}} \bar{u}(g)$ for a unique $\bar{u}: C^{1}\left(G, H^{1}\left(H, \mathbb{C}^{\times}\right)\right)$. Furthermore, the condition $(\dagger)$ gives $\bar{u}(g h)=c_{h}^{-1} \bar{u}(g) c_{h}=\bar{u}(g)$, i.e., $\bar{u} \in C^{1}\left(K, H^{1}\left(H, \mathbb{C}^{\times}\right)\right)$. Easy calculation shows that $\bar{b}^{\prime}\left(g, g^{\prime}\right)=\bar{b}\left(g, g^{\prime}\right) \bar{u}\left(g^{\prime}\right)^{-1}\left(\rho_{g^{\prime}} \bar{u}(g)\right)^{-1} \bar{u}\left(g g^{\prime}\right)$. Then $b=b^{\prime} \delta u$ where $u(g)=\bar{u}\left(g^{-1}\right)$.

Next, we prove (iii). Assuming that $[b]=0$ in $H^{2}\left(K, H^{1}\left(H, \mathbb{C}^{\times}\right)\right)$. Then there exists an $u \in C^{1}\left(G, H^{1}\left(H, \mathbb{C}^{\times}\right)\right)$, such that $u(g h)=u(g)$ for $h \in H$ and $b\left(g, g^{\prime}\right)=$ $\rho_{g^{-1}}\left(u\left(g^{\prime}\right)\right) u(g) u\left(g g^{\prime}\right)^{-1}$. Define a new family of liftings by ${\tilde{\rho_{g}}}^{\prime}=\tilde{\rho}_{g} u\left(g^{-1}\right)$. By the calculation made in (ii), $\widetilde{\rho_{g h}}{ }^{\prime}={\widetilde{\rho_{g}}}^{\prime} c_{h}$ and $\widetilde{\rho_{g g^{\prime}}}{ }^{\prime}={\widetilde{\rho_{g}}}^{\prime} \widetilde{\rho_{g^{\prime}}}{ }^{\prime}$.

Next, we prove (iv). Assuming that there exists at least one lifting. Let $\tilde{\rho}, \tilde{\rho}^{\prime}$ be two liftings. Then we can write $\tilde{\rho}_{g}^{\prime}=\tilde{\rho}_{g} \bar{u}(g)$ with $\bar{u}(g) \in H^{1}\left(H, \mathbb{C}^{\times}\right), \bar{u}(g h)=\bar{g}$. It is clear that $\bar{u}\left(g g^{\prime}\right)=\rho_{g^{\prime}}(\bar{u}(g)) \bar{u}\left(g^{\prime}\right)$. Therefore, if we define $u(g)=\bar{u}\left(g^{-1}\right)$, then $u \in Z^{1}\left(G, H^{1}\left(H, \mathbb{C}^{\times}\right)\right)$. Using the fact that $u(h g)=u(g), u \in Z^{1}\left(K, H^{1}\left(H, \mathbb{C}^{\times}\right)\right)$.

It remains to prove $(\mathrm{v})$. We choose for any $g \in G$, a lifting $\widetilde{\rho_{g}}: \widehat{H} \rightarrow \widehat{H}$ satisfying $(\dagger)$. We choose a section $s: K \rightarrow G$. Then any element $g \in G$ could be uniquely written as $\mathfrak{s}(k) h$ for $h \in H$ and $k \in K$. For $k, k^{\prime} \in K$, denote

$$
t\left(k, k^{\prime}\right)=s\left(k k^{\prime}\right)^{-1} s(k) s\left(k^{\prime}\right) \in H
$$

We also choose a section $H \rightarrow \widehat{H}$. That is, for every $h \in H$, we choose a particular lifting in $\widehat{H}$, which is denoted by $\tilde{h}$ or $(h, 1)$. Now we define $\tilde{a} \in C^{2}\left(G, \mathbb{C}^{\times}\right)$by

$$
\tilde{a}\left(s(k) h, s\left(k^{\prime}\right) h^{\prime}\right)=\frac{\widetilde{t\left(k, k^{\prime}\right)} \widetilde{\rho_{s\left(k^{\prime}\right)^{-1}}}(\tilde{h}) \tilde{h}^{\prime}}{\left(t\left(k, k^{\prime}\right) \rho_{s\left(k^{\prime}\right)^{-1}}(h) h^{\prime}, 1\right)}
$$

It is clear that the restriction of $\tilde{a}$ to $H$ is a cocycle representing the cohomology class $a$.

We claim $\delta_{G}(\tilde{a}) \in F^{2} C^{3}\left(G, \mathbb{C}^{\times}\right)$. First, according to the proof of [HS], Theorem I (or direct calculation), $\delta_{G}(\tilde{a}) \in F^{1} C^{3}\left(G, \mathbb{C}^{\times}\right)$. Therefore, it is enough to show that

$$
\frac{\tilde{a}\left(s\left(k_{2}\right) h_{2}, s\left(k_{3}\right)\right) \tilde{a}\left(s\left(k_{1}\right) h_{1}, s\left(k_{2}\right) h_{2} s\left(k_{3}\right)\right)}{\tilde{a}\left(s\left(k_{1}\right) h_{1} s\left(k_{2}\right) h_{2}, s\left(k_{3}\right)\right) \tilde{a}\left(s\left(k_{1}\right) h_{1}, s\left(k_{2}\right) h_{2}\right)}=\frac{\tilde{a}\left(s\left(k_{2}\right), s\left(k_{3}\right)\right) \tilde{a}\left(s\left(k_{1}\right) h_{1}, s\left(k_{2}\right) s\left(k_{3}\right)\right)}{\tilde{a}\left(s\left(k_{1}\right) h_{1} s\left(k_{2}\right), s\left(k_{3}\right)\right) \tilde{a}\left(s\left(k_{1}\right) h_{1}, s\left(k_{2}\right)\right)}
$$


Direct calculation shows the above identity is equivalent to

$$
\widetilde{\rho_{s\left(k_{3}\right)^{-1}}}\left(\frac{\left(h_{2}, 1\right)\left(t\left(k_{1}, k_{2}\right) \rho_{s\left(k_{2}\right)^{-1}}\left(h_{1}\right), 1\right)}{\left(t\left(k_{1}, k_{2}\right) \rho_{s\left(k_{2}\right)^{-1}}\left(h_{1}\right) h_{2}, 1\right)}\right)=\frac{\left(h_{2}, 1\right)\left(t\left(k_{1}, k_{2}\right) \rho_{s\left(k_{2}\right)^{-1}}\left(h_{1}\right), 1\right)}{\left(t\left(k_{1}, k_{2}\right) \rho_{s\left(k_{2}\right)^{-1}}\left(h_{1}\right) h_{2}, 1\right)}
$$

But this follows from $\frac{\left(h_{2}, 1\right)\left(t\left(k_{1}, k_{2}\right) \rho_{s\left(k_{2}\right)-1}\left(h_{1}\right), 1\right)}{\left(t\left(k_{1}, k_{2}\right) \rho_{\left.s\left(k_{2}\right)^{-1}\left(h_{1}\right) h_{2}, 1\right)}\right.}$ is in the central $\mathbb{C}^{\times} \subset \widehat{H}$ and $\widetilde{\rho_{s\left(k_{3}\right)^{-1}}}$ leaves the central $\mathbb{C}^{\times}$invariant.

Now, by the construction of the spectral sequence, a cocycle representing $d_{2}(a) \in$ $H^{2}\left(K, H^{1}\left(H, \mathbb{C}^{\times}\right)\right)$can be chosen as $\delta_{G}(\tilde{a})\left(h, s(k), s\left(k^{\prime}\right)\right)$ where $h \in H, k, k^{\prime} \in K$. Let us calculate this cocycle. We have

$$
\begin{aligned}
& \delta_{G}(\tilde{a})\left(h, s(k), s\left(k^{\prime}\right)\right)=\frac{\tilde{a}\left(s(k), s\left(k^{\prime}\right)\right) \tilde{a}\left(h, s(k) s\left(k^{\prime}\right)\right)}{\tilde{a}\left(h s(k), s\left(k^{\prime}\right)\right) \tilde{a}(h, s(k))} \\
& =\frac{\widetilde{\rho_{s\left(k k^{\prime}\right)^{-1}}}(\tilde{h}) \widehat{t\left(k, k^{\prime}\right)}}{\widehat{t\left(k, k^{\prime}\right)} \widetilde{\rho_{s\left(k^{\prime}\right)^{-1}}}\left(\left(\rho_{s(k)^{-1}}(h), 1\right)\right)} \frac{\left(\rho_{s(k)^{-1}}(h), 1\right)}{\widetilde{\rho_{s(k)^{-1}}}(\tilde{h})} \\
& =\frac{\widetilde{\rho_{s\left(k k^{\prime}\right)^{-1}}}(\tilde{h}) t \widetilde{t\left(k, k^{\prime}\right)}}{\widetilde{t\left(k, k^{\prime}\right)} \widetilde{\rho_{s\left(k^{\prime}\right)^{-1}}}\left(\left(\rho_{s(k)^{-1}}(h), 1\right)\right)} \widetilde{\rho_{s\left(k^{\prime}\right)^{-1}}}\left(\frac{\left(\rho_{s(k)^{-1}}(h), 1\right)}{\widetilde{\rho_{s(k)^{-1}}(\tilde{h})}}\right) \\
& =\frac{\widetilde{\rho_{s\left(k k^{\prime}\right)^{-1}}}(\tilde{h}) \widehat{t\left(k, k^{\prime}\right)}}{\widehat{t\left(k, k^{\prime}\right)} \widetilde{\rho_{s\left(k^{\prime}\right)^{-1}}}\left(\widetilde{\rho_{s(k)^{-1}}}(\tilde{h})\right)} \\
& =\widetilde{\rho_{s\left(k^{\prime}\right)^{-1}}}\left(\widetilde{\rho_{s(k)^{-1}}}\left(b\left(k, k^{\prime}\right)(h)\right)\right)=b\left(k, k^{\prime}\right)(h)
\end{aligned}
$$

Therefore, $[b]=d_{2}(a)$. This completes the proof.

5.1.3. Gerbal pairs and gerbal representations. We still assume that we have the short exact sequences of groups (5.1) and (5.2). For a character $\lambda: \mathbb{C}^{\times} \rightarrow \mathbb{C}^{\times}$, denote by $\operatorname{Rep}_{\lambda}(\widehat{H})$ the category of complex representations of $\widehat{H}$, on which the central $\mathbb{C}^{\times}$acts by $\lambda$.

We assume that the lifting $\tilde{\rho}$ of Definition 5.1 exists. Then, by Proposition 5.2 , $a \in H^{2}\left(H, \mathbb{C}^{\times}\right)^{K}$ and $d_{2}(a)=0$. Let us choose such a lifting, so that $(G, \widehat{H})$ is a gerbal pair of groups in the sense of Definition [5.1. Then we have an action of $G$ on $\widehat{H}$ by automorphisms, which leaves the central $\mathbb{C}^{\times} \subset \widehat{H}$ invariant. Therefore we obtain a representation of $G$ on $\operatorname{Rep}_{\lambda}(\widehat{H})$ (see Example 2.7). We claim that we obtain a gerbal representation of $K$ on $\operatorname{Rep}_{\lambda}(\widehat{H})$, in the sense of Definition 2.8.

Indeed, for any $g \in G$, we have an auto-equivalence $F_{g}: \operatorname{Rep}_{\lambda}(\widehat{H}) \rightarrow \operatorname{Rep}_{\lambda}(\widehat{H})$. For $h \in H$, since the action $\tilde{\rho}_{h}$ on $\widehat{H}$ is just the conjugation $c_{h}$, we have $F_{h} \cong$ id. Now we choose a set-theoretic section $s: K \rightarrow G$, and for any $k \in K$, define $F_{k}=$ $F_{s(k)}: \operatorname{Rep}_{\lambda}(\widehat{H}) \rightarrow \operatorname{Rep}_{\lambda}(\widehat{H})$. Since for $k, k^{\prime} \in K$, there exists some $h \in H$ such that $s\left(k k^{\prime}\right)=s(k) s\left(k^{\prime}\right) h$, we obtain that

$$
F_{k k^{\prime}}=F_{s\left(k k^{\prime}\right)}=F_{s(k) s\left(k^{\prime}\right) h} \cong F_{s(k)} F_{s\left(k^{\prime}\right)} F_{h} \cong F_{k} F_{k^{\prime}} .
$$

By Theorem 2.10, there is a cohomology class $e_{\tilde{\rho}} \in H^{3}\left(K, \mathcal{Z}\left(\operatorname{Rep}_{\lambda}(\widehat{H})\right)^{\times}\right)$. Observe that since $\operatorname{Rep}_{\lambda}(\widehat{H})$ is $\mathbb{C}$-linear, $\mathbb{C}^{\times} \subset \mathcal{Z}\left(\operatorname{Rep}_{\lambda}(\widehat{H})\right)^{\times}$naturally. We therefore obtain a $\operatorname{map} \mathbb{C}^{\times} \stackrel{\lambda}{\rightarrow} \mathbb{C}^{\times} \subset \mathcal{Z}\left(\operatorname{Rep}_{\lambda}(\widehat{H})\right)^{\times}$. 
Proposition 5.3. (i) $e_{\tilde{\rho}} \in \operatorname{Im}\left(H^{3}\left(K, \mathbb{C}^{\times}\right) \stackrel{\lambda_{*}}{\rightarrow} H^{3}\left(K, \mathcal{Z}\left(\operatorname{Rep}_{\lambda}(\widehat{H})\right)^{\times}\right)\right.$.

(ii) The projection of $e_{\tilde{\rho}}$ along

$$
H^{3}\left(K, \mathbb{C}^{\times}\right) \cong E_{2}^{3,0} \rightarrow H^{3}\left(K, \mathbb{C}^{\times}\right) / d_{2}\left(H^{1}\left(K, H^{1}\left(H, \mathbb{C}^{\times}\right)\right)\right) \cong E_{3}^{3,0}
$$

is independent of the choice of the lifting $\tilde{\rho}$ and is equal to $d_{3}(a)$, where $d_{3}$ is the differential $E_{3}^{0,2} \rightarrow E_{3}^{3,0}$ in the $E_{3}$ term of the Lyndon-Hochschild-Serre spectral sequence (this is well-defined since $d_{2}(a)=0$ ).

Proof. Without loss of generality, we may assume that $\lambda$ is the identity.

Fix a lifting $\tilde{\rho}$. We choose a set-theoretic section $s: K \rightarrow G$. For $k, k^{\prime} \in K$, denote

$$
t\left(k, k^{\prime}\right):=s\left(k k^{\prime}\right)^{-1} s(k) s\left(k^{\prime}\right) \in H .
$$

Recall that the functor $F_{k}: \operatorname{Rep}_{\lambda}(\widehat{H}) \rightarrow \operatorname{Rep}_{\lambda}(\widehat{H})$ is defined as follows. Let $(M, m)$ be a representation of $\widehat{H}$. Then $F_{k}(M, m)$ has $M$ as the underlying vector space with multiplication given by

$$
F_{k} m(\widehat{h}, x)=m\left(\tilde{\rho}_{s(k)^{-1}} \widehat{h}, x\right) .
$$

It is easy to show that the natural transform $c\left(k, k^{\prime}\right): F_{k} F_{k^{\prime}} M \cong F_{k k^{\prime}} M$ will satisfy the following formula



for any $x \in M, \widehat{h} \in \widehat{H}$. Therefore, we can choose

$$
\left.c\left(k, k^{\prime}\right)=m\left(\widetilde{t\left(k, k^{\prime}\right.}\right), \cdot\right),
$$

where $\widetilde{t\left(k, k^{\prime}\right)}$ is any lifting of $t\left(k, k^{\prime}\right)$ to $\widehat{H}$. The natural transformation

$$
F_{k_{1}} F_{k_{2}} F_{k_{3}} M \rightarrow F_{k_{1} k_{2}} F_{k_{3}} M \rightarrow F_{k_{1} k_{2} k_{3}} M
$$

is given by

$$
\left.x \mapsto m\left(t \widetilde{\left(k_{1} k_{2}, k_{3}\right.}\right)\left(\tilde{\rho}_{s\left(k_{3}\right)^{-1}}\left(\widetilde{t\left(\widetilde{k_{1}, k_{2}}\right)}\right)\right), x\right) .
$$

On the other hand, the natural transformation

$$
F_{k_{1}} F_{k_{2}} F_{k_{3}} M \rightarrow F_{k_{1}} F_{k_{2} k_{3}} M \rightarrow F_{k_{1} k_{2} k_{3}} M
$$

is given by

$$
\left.\left.x \mapsto m\left(t \widetilde{\left(k_{1}, k_{2} k_{3}\right.}\right) t \widetilde{\left(k_{2}, k_{3}\right.}\right), x\right) .
$$

Observe that

$$
\left.\left.i \circ \pi\left(t\left(\widetilde{k_{1}, k_{2} k_{3}}\right) t \widetilde{\left(k_{2}, k_{3}\right.}\right)\left(t\left(\widetilde{k_{1} k_{2}, k_{3}}\right) \tilde{\rho}_{s\left(k_{3}\right)^{-1}}\left(\widetilde{t\left(k_{1}, k_{2}\right.}\right)\right)\right)^{-1}\right)=1 .
$$

Therefore

$$
\left.t\left(\widetilde{k_{1}, k_{2} k_{3}}\right) t \widetilde{\left(k_{2}, k_{3}\right.}\right)\left(t\left(\widetilde{k_{1} k_{2}, k_{3}}\right) \tilde{\rho}_{s\left(k_{3}\right)^{-1}}\left(\widetilde{\left.t\left(\widetilde{k_{1}, k_{2}}\right)\right)}\right)^{-1}\right.
$$

is in the central $\mathbb{C}^{\times}$of $\widehat{H}$.

We find that

$$
\left.\left.\left.e_{\tilde{\rho}}\left(k_{1}, k_{2}, k_{3}\right)=t\left(\widetilde{k_{1}, k_{2} k_{3}}\right) t \widetilde{\left(k_{2}, k_{3}\right.}\right)\left(t \widetilde{k_{1} k_{2}, k_{3}}\right) \tilde{\rho}_{s\left(k_{3}\right)^{-1}}\left(\widetilde{t\left(k_{1}, k_{2}\right.}\right)\right)\right)^{-1}
$$

is a 3 -cocycle. Different choices of liftings $\widetilde{t\left(k, k^{\prime}\right)}$ make $e_{\tilde{\rho}}$ differ by a coboundary. Therefore, $e_{\tilde{\rho}}$ is a well-defined cohomology class in $H^{3}\left(K, \mathbb{C}^{\times}\right)$. Furthermore, if $\tilde{\rho}^{\prime}$ is 
another lifting of $\rho$, then we know from the proof of Lemma 5.2 that $\tilde{\rho}_{g}^{\prime}=\tilde{\rho}_{g} u\left(g^{-1}\right)$, where $u \in Z^{1}\left(K, H^{1}\left(H, \mathbb{C}^{\times}\right)\right)$. Therefore, we have

$$
\frac{e_{\tilde{\rho}^{\prime}}\left(k_{1}, k_{2}, k_{3}\right)}{e_{\tilde{\rho}}\left(k_{1}, k_{2}, k_{3}\right)}=\frac{1}{u\left(s\left(k_{3}\right)\right)\left(t\left(k_{2}, k_{3}\right)\right)} .
$$

Applying the same method as in the proof of Lemma 5.2, one can show the right hand side is an expression for $d_{2}(u)$. Therefore, the image of $e_{\tilde{\rho}}$ in

$$
H^{3}\left(K, \mathbb{C}^{\times}\right) / d_{2}\left(H^{1}\left(K, H^{1}\left(H, \mathbb{C}^{\times}\right)\right)\right)
$$

is independent of the lifting $\tilde{\rho}$.

It remains to prove that this image is exactly the class $d_{3}(a)$. As in the proof of Lemma 5.2, we choose a section $H \rightarrow \widehat{H}$, and the corresponding lifting of $h$ is denoted by $\tilde{h}$ or $(h, 1)$. Then we define

$$
\tilde{a}\left(s(k) h, s\left(k^{\prime}\right) h^{\prime}\right)=\frac{\widetilde{t\left(k, k^{\prime}\right)} \tilde{\rho}_{s\left(k^{\prime}\right)^{-1}}(\tilde{h}) \tilde{h}^{\prime}}{\left(t\left(k, k^{\prime}\right) \rho_{s\left(k^{\prime}\right)^{-1}}(h) h^{\prime}, 1\right)}
$$

We have already shown that $\delta_{G}(a) \in F^{2} C^{3}\left(G, \mathbb{C}^{\times}\right)$. Using the fact that $\tilde{\rho}_{g g^{\prime}}=\tilde{\rho}_{g} \tilde{\rho}_{g^{\prime}}$, one can even show that $\delta_{G}(a) \in F^{3} C^{3}\left(G, \mathbb{C}^{\times}\right)$. Furthermore, a direct calculation shows that

$$
\delta_{G}(\tilde{a})\left(s\left(k_{1}\right), s\left(k_{2}\right), s\left(k_{3}\right)\right)=e_{\tilde{\rho}}\left(k_{1}, k_{2}, k_{3}\right) .
$$

This completes the proof.

Observe that to prove the above proposition, we do not really use the fact that each object in $\operatorname{Rep}_{\lambda}(\widehat{H})$ is realized as a representation of $\widehat{H}$. All we need is that $\operatorname{Rep}_{\lambda}(\widehat{H})$ is a $\mathbb{C}$-linear abelian category, with a genuine action of $G$ satisfying certain properties. This allows us to generalize the proposition in the following way.

We still assume that $(G, \widehat{H})$ is a gerbal pair. Therefore,there is an action $\tilde{\rho}$ of $G$ on $\widehat{H}$, which lifts the action $\rho$ of $G$ on $H$. Recall that $\pi: \widehat{H} \rightarrow H$ so that for any $h \in H$, $\pi^{-1}(h)$ is a $\mathbb{C}^{\times}$-torsor. Let $\mathcal{C}$ be a $\mathbb{C}$-linear abelian category. Recall the definition of the 2-group $\mathbb{G} \mathbb{L}(\mathcal{C})$ (see 2.3 .2 ). Assume that there is a genuine representation of $G$ on $\mathcal{C}$, i.e. a 2-group homomorphism $F: G \rightarrow \mathbb{G} \mathbb{L}(\mathcal{C})$. For simplicity, we will assume that $F_{g} F_{g^{\prime}}=F_{g g^{\prime}}$. (This is not essential, but simplifies the discussion.) Since $\mathcal{C}$ is $\mathbb{C}$-linear, there is a natural $\mathbb{C}^{\times}$-action on $\operatorname{Hom}_{\mathbb{G L}(\mathcal{C})}\left(F_{g}, \mathbf{1}_{\mathcal{C}}\right)$, for any $g \in G$. Assume that for any $h \in H$, there is a $\mathbb{C}^{\times}$-equivariant embedding

$$
\alpha_{h}: \pi^{-1}(h) \rightarrow \operatorname{Hom}_{\mathbb{G} \mathbb{L}(\mathcal{C})}\left(F_{h}, \mathbf{1}_{\mathcal{C}}\right)
$$

such that:

(i) for any $g \in G$, the following diagram is commutative:

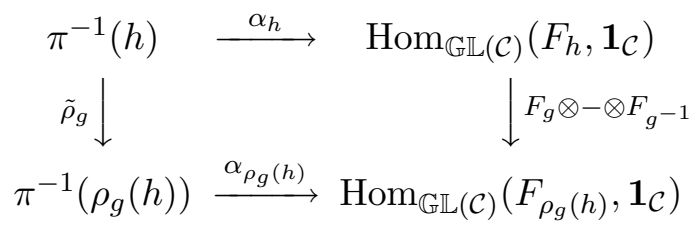


(ii) for any $h, h^{\prime} \in H$, the following diagram is commutative:

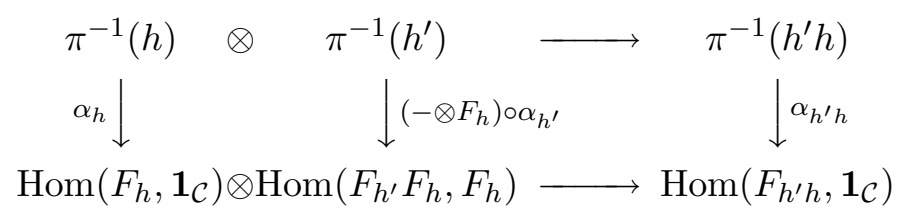

Proposition 5.4. Assumptions are as above. Then there is a gerbal representation of $K$ on $\mathcal{C}$. The corresponding cohomology class $e \in \operatorname{Im}\left(H^{3}\left(K, \mathbb{C}^{\times}\right) \rightarrow H^{3}\left(K, \mathcal{Z}(\mathcal{C})^{\times}\right)\right.$. The projection of e along

$$
H^{3}\left(K, \mathbb{C}^{\times}\right) \cong E_{2}^{3,0} \rightarrow H^{3}\left(K, \mathbb{C}^{\times}\right) / d_{2}\left(H^{1}\left(K, H^{1}\left(H, \mathbb{C}^{\times}\right)\right)\right) \cong E_{3}^{3,0}
$$

is equal to $d_{3}(a)$.

The proof is identical to the proof of Proposition 5.3 ,

5.2. Gerbal pairs associated with $G L_{\infty, \infty}$. Having developed the general formalism of gerbal pairs, we now apply it to a special case. Namely, we show that there is an action of $\widetilde{G L}_{\infty}\left(\mathfrak{g l}_{\infty}\right)$ on $\widehat{G L}_{\mathfrak{f}, \infty}$, so that the groups $\left(\widetilde{G L}_{\infty}\left(\mathfrak{g l}_{\infty}\right), \widehat{G L}_{\mathfrak{f}, \infty}\right)$, equipped with the short exact sequences (4.5) and (4.9), form a gerbal pair in the sense of Definition [5.1. Similarly, $\left(\widetilde{G L}_{\infty}\left(\mathfrak{g l}_{\infty}\right), \widehat{G L}_{\mathfrak{f}, \infty}^{0}\right)$ equipped with (4.4) and (4.11) also form a gerbal pair. Applying Proposition 5.2 , we will obtain a particular class $\left[E_{3}\right] \in H^{3}\left(G L_{\infty, \infty}, \mathbb{C}^{\times}\right)$, and similarly $\left[\widehat{E}_{3}\right] \in H^{3}\left(\widehat{G L}_{\infty, \infty}, \mathbb{C}^{\times}\right)$.

Recall that (4.5) gives us a map $\widehat{G L}_{\mathfrak{f}, \infty} \rightarrow G L_{\mathfrak{f}, \infty}$. Combined with (4.5), we obtain a group homomorphism $\delta: \widehat{G L}_{\mathfrak{f}, \infty} \rightarrow \widetilde{G L}_{\infty}\left(\mathfrak{g l}_{\infty}\right)$ as the composition of $\widehat{G L}_{\mathfrak{f}, \infty} \rightarrow$ $G L_{\mathfrak{f}, \infty} \rightarrow \widetilde{G L}_{\infty}\left(\mathfrak{g l}_{\infty}\right)$.

Theorem 5.5. There is a unique action of $\widetilde{G L}_{\infty}\left(\mathfrak{g l}_{\infty}\right)$ on $\widehat{G L}_{\mathfrak{f}, \infty}$, making the pair $\left(\widetilde{G L}_{\infty}\left(\mathfrak{g l}_{\infty}\right), \widehat{G L}_{\mathfrak{f}, \infty}\right)$, equipped with the short exact sequences 4.5) and 4.9), into a gerbal pair in the sense of Definition 5.1.

Remark 5.6. This action will also make $\left(\widetilde{G L}_{\infty}\left(\mathfrak{g l}_{\infty}\right), \widehat{G L}_{\mathfrak{f}, \infty}^{0}\right)$ a gerbal pair.

Proof. Once such an action exists, the uniqueness is clear. This is because according to Proposition 5.2, all such actions form a torsor under $Z^{1}\left(G L_{\infty, \infty}, H^{1}\left(G L_{\mathfrak{f}, \infty}, \mathbb{C}^{\times}\right)\right)=$ $H^{1}\left(G L_{\infty, \infty}, \mathbb{C}^{\times}\right)=0$. Therefore, we will be focusing on the existence of such an action.

Observe that $G L_{\mathfrak{f}, \infty}$ is a normal subgroup of $G L_{\infty}^{+}\left(\mathfrak{g l}_{\infty}\right)$, and the action of $\widetilde{G L}{ }_{\infty}\left(\mathfrak{g l}_{\infty}\right)$ on $G L_{\mathfrak{f}, \infty}$ factors through the adjoint action of $G L_{\infty}^{+}\left(\mathfrak{g l}_{\infty}\right)$ on $G L_{\mathfrak{f}, \infty}$ via the natural projection $\widetilde{G L}_{\infty}\left(\mathfrak{g l}_{\infty}\right) \rightarrow G L_{\infty}^{+}\left(\mathfrak{g l}_{\infty}\right)$. Therefore, it is enough to prove that there is an action of $G L_{\infty}^{+}\left(\mathfrak{g l}_{\infty}\right)$ on $\widehat{G L}_{\mathfrak{f}, \infty}$ such that $\left(G L_{\infty}^{+}\left(\mathfrak{g l}_{\infty}\right), \widehat{G L}_{\mathfrak{f}, \infty}\right)$ is a gerbal pair.

Denote the conjugation of $G L_{\infty}^{+}\left(\mathfrak{g l}_{\infty}\right)$ on $G L_{\mathfrak{f}, \infty}$ by $\rho$, i.e., $\rho_{g}(h)=g h g^{-1}$ for $g \in$ $G L_{\infty}^{+}\left(\mathfrak{g l}_{\infty}\right), h \in G L_{\mathfrak{f}, \infty}$. Denote the conjugation of $G L_{\mathfrak{f}, \infty}$ on $\widehat{G L}_{\mathfrak{f}, \infty}$ by $c$, i.e., $c_{h}(a)=$ $\tilde{h} a \tilde{h}^{-1}$ for $h \in G L_{\mathfrak{f}, \infty}, a \in \widehat{G L}_{\mathfrak{f}, \infty}$, and $\tilde{h} \in \widehat{G L}_{\mathfrak{f}, \infty}$ is any lifting of $h$. We want to show that $\rho$ lifts to an action $\tilde{\rho}$ of $G L_{\infty}^{+}\left(\mathfrak{g l}_{\infty}\right)$ on $\widehat{G L}_{\mathfrak{f}, \infty}$, such that: (i) $\left.\tilde{\rho}\right|_{G L_{\mathfrak{f}, \infty}}=c$; (ii) for any $g \in G L_{\infty}^{+}\left(\mathfrak{g l}_{\infty}\right), \tilde{\rho}_{g}$ is the identity map on the central $\mathbb{C}^{\times} \subset G L_{\mathfrak{f}, \infty}$.

First, by Corollary 4.14, for any $g \in G L_{\infty}^{+}\left(\mathfrak{g l}_{\infty}\right), \rho_{g}: G L_{\mathfrak{f}, \infty} \rightarrow G L_{\mathfrak{f}, \infty}$ lifts to a group automorphism $\widetilde{\rho_{g}}: \widehat{G L}_{\mathfrak{f}, \infty} \rightarrow \widehat{G L}_{\mathfrak{f}, \infty}$, which leaves the central $\mathbb{C}^{\times}$invariant. We denote the restriction of $\widetilde{\rho_{g}}$ to $\widehat{G L}_{\mathfrak{f}, \infty}^{0}$ by ${\widetilde{\rho_{g}}}^{0}$. Since $H^{1}\left(G L_{\mathfrak{f}, \infty}^{0}, \mathbb{C}^{\times}\right)=0$, such ${\widetilde{\rho_{g}}}^{0}$ is unique. 
Therefore, we obtain an action $\tilde{\rho}^{0}$ of $G L_{\infty}^{+}\left(\mathfrak{g l}_{\infty}\right)$ on $G L_{\mathfrak{f}, \infty}^{0}$ that lifts $\rho$. It is clear that this action makes $\widehat{G L}_{\mathfrak{f}, \infty}^{0} \rightarrow G L_{\infty}^{+}\left(\mathfrak{g l}_{\infty}\right)$ a gerbal pair.

Next, we show we can extend $\tilde{\rho}^{0}$ to an action $\tilde{\rho}$ of $G L_{\infty}^{+}\left(\mathfrak{g l}_{\infty}\right)$ on $\widehat{G L}_{\mathfrak{f}, \infty}$ such that $\widehat{G L}_{\mathfrak{f}, \infty} \rightarrow G L_{\infty}^{+}\left(\mathfrak{g l}_{\infty}\right)$ is a gerbal pair. Recall that by choosing an element $\sigma \in G L_{\mathfrak{f}, \infty}$ such that $\operatorname{deg}(\sigma)=1$, we obtain a splitting $G L_{\mathfrak{f}, \infty}=G L_{\mathfrak{f}, \infty}^{0} \rtimes \mathbb{Z}$. For example, we will choose $\sigma$ such that $\sigma\left(t^{i}\right)=t^{i+1}$ and $\sigma\left(s^{i} t^{j}\right)=s^{i} t^{j}$ for $i \geq 1$. Choosing a lifting $\widehat{\sigma}$ of $\sigma$ in $\widehat{G L}_{\mathfrak{f}, \infty}$, we obtain a splitting $\widehat{G L}_{\mathfrak{f}, \infty}=\widehat{G L}_{\mathfrak{f}, \infty}^{0} \rtimes \mathbb{Z}$. Therefore, we denote elements in $\widehat{G L}_{\mathfrak{f}, \infty}$ by $\left(a, \widehat{\sigma}^{m}\right)$ for $a \in \widehat{G L}_{\mathfrak{f}, \infty}^{0}$ and the multiplication by $\left(a, \widehat{\sigma}^{m}\right)\left(a^{\prime}, \widehat{\sigma}^{m^{\prime}}\right)=$ $\left(a \widehat{\sigma}^{m} a^{\prime} \widehat{\sigma}^{-m}, \widehat{\sigma}^{m+m^{\prime}}\right)$.

For any $g \in G L_{\infty}^{+}\left(\mathfrak{g l}_{\infty}\right)$, we choose a lifting $\widetilde{\rho_{g}(\sigma)} \in \widehat{G L}_{\mathfrak{f}, \infty}$ of $\rho_{g}(\sigma) \in G L_{\mathfrak{f}, \infty}$. We check that ${\widetilde{\rho_{g}}}_{:}: \widehat{G L}_{\mathfrak{f}, \infty} \rightarrow \widehat{G L}_{\mathfrak{f}, \infty}$ defined by

$$
\widetilde{\rho}_{g}\left(a, \widehat{\sigma}^{m}\right)=\left(\tilde{\rho}_{g}^{0}(a){\widetilde{\rho_{g}(\sigma)}}^{m} \widehat{\sigma}^{-m}, \widehat{\sigma}^{m}\right)
$$

is a group homomorphism and therefore is a lifting of $\rho_{g}$. Thus, we must check that

$$
\widetilde{\rho_{g}}\left(\left(a, \widehat{\sigma}^{m}\right)\left(a^{\prime}, \widehat{\sigma}^{m^{\prime}}\right)\right)=\widetilde{\rho_{g}}\left(\left(a, \widehat{\sigma}^{m}\right)\right) \widetilde{\rho_{g}}\left(\left(a^{\prime}, \widehat{\sigma}^{m^{\prime}}\right)\right) .
$$

By definition,

$$
\begin{aligned}
& \text { l.h.s. }=\widetilde{\rho}_{g}\left(\left(a \widehat{\sigma}^{m} a^{\prime} \widehat{\sigma}^{-m}, \widehat{\sigma}^{m+m^{\prime}}\right)\right) \\
& =\left(\tilde{\rho}_{g}^{0}\left(a \widehat{\sigma}^{m} a^{\prime} \widehat{\sigma}^{-m}{\widehat{\rho_{g}(\sigma)}}^{m+m^{\prime}} \widehat{\sigma}^{-m-m^{\prime}}, \widehat{\sigma}^{m+m^{\prime}}\right)\right. \\
& =\left(\tilde{\rho}_{g}^{0}(a) \tilde{\rho}_{g}^{0}\left(\widehat{\sigma}^{m} a^{\prime} \widehat{\sigma}^{-m}\right){\widehat{\rho_{g}(\sigma)}}^{m+m^{\prime}} \widehat{\sigma}^{-m-m^{\prime}}, \widehat{\sigma}^{m+m^{\prime}}\right) \text {. } \\
& \text { r.h.s. }=\left(\tilde{\rho}_{g}^{0}(a){\widetilde{\rho_{g}(\sigma)}}^{m} \widehat{\sigma}^{-m}, \widehat{\sigma}^{m}\right)\left(\tilde{\rho}_{g}^{0}\left(a^{\prime}\right){\widetilde{\rho_{g}(\sigma)}}^{m^{\prime}} \widehat{\sigma}^{-m^{\prime}}, \widehat{\sigma}^{m^{\prime}}\right) \\
& =\left(\tilde{\rho}_{g}^{0}(a){\widetilde{\rho_{g}(\sigma)}}^{m} \widehat{\sigma}^{-m} \widehat{\sigma}^{m} \tilde{\rho}_{g}^{0}\left(a^{\prime}\right){\widetilde{\rho_{g}(\sigma)}}^{m^{\prime}} \widehat{\sigma}^{-m^{\prime}} \widehat{\sigma}^{-m}, \widehat{\sigma}^{m+m^{\prime}}\right) \\
& =\left(\tilde{\rho}_{g}^{0}(a){\widetilde{\rho_{g}(\sigma)}}^{m} \tilde{\rho}_{g}^{0}\left(a^{\prime}\right){\widetilde{\rho_{g}(\sigma)}}^{-m}{\widetilde{\rho_{g}(\sigma)}}^{m+m^{\prime}} \widehat{\sigma}^{-m-m^{\prime}}, \widehat{\sigma}^{m+m^{\prime}}\right) \text {. }
\end{aligned}
$$

Therefore, we must check that

$$
\tilde{\rho}_{g}^{0}\left(\widehat{\sigma}^{m} a^{\prime} \widehat{\sigma}^{-m}\right)={\widetilde{\rho_{g}(\sigma)}}^{m} \tilde{\rho}_{g}^{0}\left(a^{\prime}\right){\widetilde{\rho_{g}(\sigma)}}^{-m} .
$$

However, observe the two group homomorphisms $\widehat{G L}_{\mathfrak{f}, \infty}^{0} \rightarrow \widehat{G L}_{\mathfrak{f}, \infty}^{0}$ given by $a^{\prime} \mapsto$ $\tilde{\rho}_{g}^{0}\left(\widehat{\sigma}^{m} a^{\prime} \widehat{\sigma}^{-m}\right)$ and $a^{\prime} \mapsto{\widetilde{\rho_{g}(\sigma)}}^{m} \tilde{\rho}_{g}^{0}\left(a^{\prime}\right){\widetilde{\rho_{g}(\sigma)}}^{-m}$ are liftings of the group homomorphism $G L_{\mathfrak{f}, \infty}^{0} \rightarrow G L_{\mathfrak{f}, \infty}^{0}$ given by $a \mapsto \rho_{g}\left(\sigma^{m} a \sigma^{-m}\right)$. Since $H^{1}\left(G L_{\mathfrak{f}, \infty}^{0}, \mathbb{C}^{\times}\right)=0$, the two homomorphisms must be the same. Therefore, (5.4) holds and $\widetilde{\rho_{g}}$ defined as above is a group homomorphism.

To finish the proof, we will show that for each $g \in G L_{\infty}^{+}\left(\mathfrak{g l}_{\infty}\right)$, we can choose a particular lifting $\widetilde{\rho_{g}(\sigma)}$ so that $\widetilde{\rho_{g}}=c_{g}$ is just conjugation by $g$ if $g \in G L_{\mathfrak{f}, \infty}$ and $\widetilde{\rho_{g g^{\prime}}}=$ $\widetilde{\rho_{g}} \widetilde{\rho_{g^{\prime}}}$. To this end, we make use the following two claims. Recall that $g \in G L_{\infty}^{+}\left(\mathfrak{g l}_{\infty}\right)$ acts on $\mathcal{O}_{\mathbb{K}}$ by formula (4.8). We will write $\mathcal{O}_{\mathbb{K}}=\mathbb{C}((t)) \oplus s \mathcal{O}_{\mathbb{K}}$.

(i) For any $g \in G L_{\infty}^{+}\left(\mathfrak{g l}_{\infty}\right)$, there exists some $h \in G L_{\mathfrak{f}, \infty}^{0}$ such that $\left.g\right|_{\mathbb{C}((t))}=\left.h\right|_{\mathbb{C}((t))}$ and $\rho_{g}(\sigma)=\rho_{h}(\sigma)$.

(ii)For $h, h^{\prime} \in G L_{\mathfrak{f}, \infty}^{0}$, if $\left.h\right|_{\mathbb{C}((t))}=\left.h^{\prime}\right|_{\mathbb{C}((t))}$ and $\rho_{h}(\sigma)=\rho_{h^{\prime}}(\sigma)$, then $c_{h}(\widehat{\sigma})=c_{h^{\prime}}(\widehat{\sigma})$. 
If these two claims hold, then we let $\widetilde{\rho_{g}(\sigma)}=c_{h}(\widehat{\sigma})$ for some $h \in G L_{\mathfrak{f}, \infty}^{0}$ satisfying properties in Claim (i). By Claim (ii), this is well-defined. We check that

$$
\widetilde{\rho_{g g^{\prime}}}\left(\left(a, \widehat{\sigma}^{m}\right)\right)=\widetilde{\rho_{g}}\left(\widetilde{\rho_{g^{\prime}}}\left(\left(a, \widehat{\sigma}^{m}\right)\right)\right) \text {. }
$$

Assume that $h, h^{\prime} \in G L_{\mathfrak{f}, \infty}^{0}$ such that $\rho_{g}(\sigma)=c_{h}(\sigma),\left.g\right|_{\mathbb{C}((t))}=\left.h\right|_{\mathbb{C}((t))}$ and $\rho_{g^{\prime}}(\sigma)=$ $c_{h^{\prime}}(\sigma),\left.g^{\prime}\right|_{\mathbb{C}((t))}=\left.h^{\prime}\right|_{\mathbb{C}((t))}$. Then $\left.g g^{\prime}\right|_{\mathbb{C}((t))}=\left.\rho_{g}\left(h^{\prime}\right) h\right|_{\mathbb{C}((t))}$ and $\rho_{g g^{\prime}}(\sigma)=c_{\rho_{g}\left(h^{\prime}\right) h}(\sigma)$. Now,

$$
\begin{aligned}
& \text { l.h.s. }=\left(\tilde{\rho}_{g g^{\prime}}^{0}(a)\left(c_{\rho_{g}\left(h^{\prime}\right) h}(\widehat{\sigma})\right)^{m} \widehat{\sigma}^{-m}, \widehat{\sigma}^{m}\right), \\
\text { r.h.s. }= & \widetilde{\rho}_{g}\left(\left(\tilde{\rho}_{g^{\prime}}^{0}(a)\left(c_{h^{\prime}}(\widehat{\sigma})\right)^{m} \widehat{\sigma}^{-m}, \widehat{\sigma}^{m}\right)\right) \\
= & \left(\tilde{\rho}_{g}^{0}\left(\tilde{\rho}_{g^{\prime}}^{0}(a)\right) \tilde{\rho}_{g}^{0}\left(\left(c_{h^{\prime}}(\widehat{\sigma})\right)^{m} \widehat{\sigma}^{-m}\right)\left(c_{h}(\widehat{\sigma})\right)^{m} \widehat{\sigma}^{-m}, \widehat{\sigma}^{m}\right) \\
= & \left(\tilde{\rho}_{g g^{\prime}}^{0}(a) \tilde{\rho}_{g}^{0}\left(\tilde{h}^{\prime} \widehat{\sigma}^{m} \tilde{h}^{\prime}-1 \widehat{\sigma}^{-m}\right)\left(c_{h}(\widehat{\sigma})\right)^{m} \widehat{\sigma}^{-m}, \widehat{\sigma}^{m}\right) \\
= & \left(\tilde{\rho}_{g g^{\prime}}^{0}(a) \tilde{\rho}_{g}^{0}\left(\tilde{h}^{\prime}\right) \tilde{\rho}_{g}^{0}\left(\widehat{\sigma}^{m} \tilde{h}^{\prime}-1 \widehat{\sigma}^{-m}\right)\left(c_{h}(\widehat{\sigma})\right)^{m} \widehat{\sigma}^{-m}, \widehat{\sigma}^{m}\right) \\
= & \left(\tilde{\rho}_{g g^{\prime}}^{0}(a) \tilde{\rho}_{g}^{0}\left(\tilde{h}^{\prime}\right)\left(c_{h}(\widehat{\sigma})\right)^{m} \tilde{\rho}_{g}^{0}\left(\tilde{h}^{\prime}-1\right) \widehat{\sigma}^{-m}, \widehat{\sigma}^{m}\right) \\
= & \text { l.r.s, since } \tilde{\rho}_{g}^{0}\left(\tilde{h}^{\prime}\right) \text { is a lifting of } \rho_{g}\left(h^{\prime}\right) .
\end{aligned}
$$

We also check that $\widetilde{\rho_{h}}=c_{h}$ for $h \in G L_{\mathfrak{f}, \infty}$. But this follows from $\tilde{\rho}_{h}^{0}(a)=c_{h}(a)$ for $h \in G L_{\mathfrak{f}, \infty}, a \in \widehat{G L}_{\mathfrak{f}, \infty}^{0}$ and our choice $\widetilde{\rho_{h}(\sigma)}$.

It remains to prove the two claims we made. We begin with the proof of Claim (i). Recall that we choose $\sigma$ such that $\sigma\left(t^{i}\right)=t^{i+1}$ and $\sigma\left(s^{i} t^{j}\right)=s^{i} t^{j}$ for $i \geq 1$. For $g \in G L_{\infty}^{+}\left(\mathfrak{g l}_{\infty}\right)$, there exists $N \in \mathbb{Z}$ such that $g^{-1} s^{N} \mathcal{O}_{\mathbb{K}} \subset s \mathcal{O}_{\mathbb{K}}$. Then one can find some Tate vector space $V \subset g s \mathcal{O}_{\mathbb{K}}$ such that $g s \mathcal{O}_{\mathbb{K}}=V \oplus s^{N} \mathcal{O}_{\mathbb{K}}$. Therefore $\mathcal{O}_{\mathbb{K}}=g \mathbb{C}((t)) \oplus g s \mathcal{O}_{\mathbb{K}}=g \mathbb{C}((t)) \oplus V \oplus s^{N} \mathcal{O}_{\mathbb{K}}$. We can define an $h: \mathcal{O}_{\mathbb{K}} \rightarrow \mathcal{O}_{\mathbb{K}}$ by $\left.h\right|_{s^{N} \mathcal{O}_{\mathbb{K}}}=\mathrm{id},\left.h\right|_{\mathbb{C}((t))}=\left.g\right|_{\mathbb{C}((t))}$ and $h\left(\sum_{i=1}^{N-1} s^{i} \mathbb{C}((t))\right)=V$. It is clear that $h \in G L_{\mathfrak{f}, \infty}$ and $g \sigma g^{-1}=h \sigma h^{-1}$. Now we choose some $h_{1} \in G L_{\mathfrak{f}, \infty}$, such that $\operatorname{deg}(h)=\operatorname{deg}\left(h_{1}\right)$, $\left.h_{1}\right|_{\mathbb{C}((t))}=\mathrm{id}$ and $h_{1}\left(s \mathcal{O}_{\mathbb{K}}\right)=s \mathcal{O}_{\mathbb{K}}$. Since $h_{1} \sigma h_{1}^{-1}=\sigma$, we see that $h h_{1}^{-1}$ satisfies all the required properties.

Next, we prove the Claim (ii). It is enough to prove that if $h \in G L_{\mathfrak{f}, \infty}^{0},\left.h\right|_{\mathbb{C}((t))}=$ id and $\sigma h \sigma^{-1}=h$, then $\widehat{\sigma} \tilde{h} \widehat{\sigma}^{-1}=\tilde{h}$ for $\tilde{h}$ any lifting of $h$ in $\widehat{G L}_{\mathfrak{f}, \infty}^{0}$. It is easy to prove that in this case $h\left(s \mathcal{O}_{\mathbb{K}}\right)=s \mathcal{O}_{\mathbb{K}}$. Recall the definition of $G L_{\mathfrak{f}, n}\left(\mathfrak{g l}_{\infty}\right)$, which is a subgroup of $G L_{\mathfrak{f}, \infty}$ consisting of whose $a: \mathfrak{g l}_{\infty}[[s]] \rightarrow \mathfrak{g l}_{\infty}[[s]]$ such that $\left.a\right|_{s^{n}} \mathfrak{g l}_{\infty}[[s]]=$ id. Let $L_{0}=\mathbb{C}[[t]][[s]] d t d s$. Assume that $h \in G L_{\mathfrak{f}, n}^{0}\left(\mathfrak{g l}_{\infty}\right)$. Then $\tilde{h}=(h, e)$ where

$e \in \operatorname{det}\left(\frac{h L_{0}}{L_{0} \cap s^{N} \mathcal{O}_{\mathbb{K}}} \mid \frac{L_{0}}{L_{0} \cap s^{N} \mathcal{O}_{\mathbb{K}}}\right)^{\times}=\operatorname{det}\left(\frac{h L_{0}}{L_{0} \cap\left(s^{N} \mathcal{O}_{\mathbb{K}}+\mathbb{C}((t))\right)} \mid \frac{L_{0}}{L_{0} \cap\left(s^{N} \mathcal{O}_{\mathbb{K}}+\mathbb{C}((t))\right)}\right)^{\times}$

It is clear that $\sigma$ induces identity on this line. Likewise, $\widehat{\sigma}=(\sigma, o)$ where

$$
o \in \operatorname{det}\left(\frac{\sigma L_{0}}{L_{0} \cap s^{N} \mathcal{O}_{\mathbb{K}}} \mid \frac{L_{0}}{L_{0} \cap s^{N} \mathcal{O}_{\mathbb{K}}}\right)^{\times}=\operatorname{det}\left(\frac{\sigma L_{0}}{L_{0} \cap s \mathcal{O}_{\mathbb{K}}} \mid \frac{L_{0}}{L_{0} \cap s \mathcal{O}_{\mathbb{K}}}\right)^{\times}
$$

and $h$ induces identity on this line. Therefore, $\tilde{h} \widehat{\sigma}=\widehat{\sigma} \tilde{h}$.

Theorem 5.7. (i) $H^{2}\left(G L_{\infty, \infty}, \mathbb{C}^{\times}\right)=\mathbb{C}^{\times}$.

(ii) The cohomology class $\left[D_{2}\right] \in H^{2}\left(G L_{\mathfrak{f}, \infty}, \mathbb{C}^{\times}\right)$is transgressive, that is, it belongs to $H^{2}\left(G L_{\mathfrak{f}, \infty}, \mathbb{C}^{\times}\right)^{G L_{\infty, \infty}}=E_{2}^{0,2}$, and $d_{2}\left[D_{2}\right]=0$, so that $\left[D_{2}\right] \in E_{3}^{0,2}$.

(iii) $E_{3}^{3,0} \cong H^{3}\left(G L_{\infty, \infty}, \mathbb{C}^{\times}\right)$and $d_{3}\left[D_{2}\right]$ is non-zero in $H^{3}\left(G L_{\infty, \infty}, \mathbb{C}^{\times}\right)$. We denote this class by $\left[E_{3}\right]$. 
Proof. Since $H^{\bullet}\left(\widetilde{G L}_{\infty}\left(\mathfrak{g l}_{\infty}\right), \mathbb{C}^{\times}\right) \cong \mathbb{C}^{\times}$and $H^{1}\left(G L_{\mathfrak{f}, \infty}, \mathbb{C}^{\times}\right) \cong \mathbb{C}^{\times}$, (i) follows from the spectral sequence associated to (4.9). (ii) follows from Theorem 5.5] and Proposition 5.2. We shall prove (iii). Indeed, $E_{3}^{3,0} \cong H^{3}\left(G L_{\infty, \infty}, \mathbb{C}^{\times}\right)$follows from

$$
E_{2}^{1,1}=H^{1}\left(G L_{\infty, \infty}, H^{1}\left(G L_{\mathfrak{f}, \infty}, \mathbb{C}^{\times}\right)\right)=0
$$

which in turn follows from

$$
H^{1}\left(G L_{\infty, \infty}, \mathbb{C}^{\times}\right) \hookrightarrow H^{1}\left(\widetilde{G L}_{\infty}\left(\mathfrak{g l}_{\infty}\right), \mathbb{C}^{\times}\right)=0, \quad H^{1}\left(G L_{\mathfrak{f}, \infty}, \mathbb{C}^{\times}\right)=\mathbb{C}^{\times}
$$

The reason for $d_{3}\left[D_{2}\right] \neq 0$ is the same as that for $\left[C_{2}^{0}\right] \neq 0$ and $\left[D_{2}^{0}\right] \neq 0$.

We have a similar theorem for $\widehat{G L}_{\infty, \infty}$, whose proof is even simpler.

Theorem 5.8. (i) $H^{2}\left(\widehat{G L}_{\infty, \infty}, \mathbb{C}^{\times}\right)=0$.

(ii) The cohomology class $\left[D_{2}^{0}\right] \in H^{2}\left(G L_{\mathfrak{f}, \infty}^{0}, \mathbb{C}^{\times}\right)$is transgressive, that is, it belongs to $H^{2}\left(G L_{\mathrm{f}, \infty}^{0}, \mathbb{C}^{\times}\right)^{\widehat{G L}}{ }_{\infty, \infty}=E_{2}^{0,2}$, and $d_{2}\left[D_{2}^{0}\right]=0$, so that $\left[D_{2}^{0}\right] \in E_{3}^{0,2}$.

(iii) $E_{3}^{3,0} \cong H^{3}\left(\widehat{G L}_{\infty, \infty}, \mathbb{C}^{\times}\right)$and $d_{3}\left[D_{2}^{0}\right]$ is non-zero in $H^{3}\left(G L_{\infty, \infty}, \mathbb{C}^{\times}\right)$. We denote this class by $\left[\widehat{E}_{3}\right]$. Then $\left[\widehat{E}_{3}\right]$ is the pullback of $\left[E_{3}\right]$ along $\widehat{G L}_{\infty, \infty} \rightarrow G L_{\infty, \infty}$.

5.3. Computation of the cohomology classes of gerbal representations. In this section we will calculate the cohomology class corresponding to the gerbal representation of $G L_{\infty, \infty}$ on the category $\mathcal{C}_{\mathcal{O}_{\mathbb{K}}}^{\mathrm{ss}}$ of Clifford modules constructed in Theorem 3.11. We will also construct a gerbal representation of $G L_{\infty, \infty}$ on another abelian category which also gives this cohomology class.

5.3.1. The cohomology class of the gerbal representation constructed in Theorem 3.11. Let us recall that the category $\mathcal{C}_{\mathcal{O}_{\mathbb{K}}}^{\mathrm{ss}}$ defined in 3.2 .2 is a semisimple abelian category. The irreducible objects are vacuum modules $M_{L}$ over the Clifford algebra $\mathrm{Cl}_{\mathcal{O}_{\mathbb{K}}}$. They are labeled by secondary lattices $L \subset \mathcal{O}_{\mathbb{K}}$. Let us also recall that $\operatorname{Hom}_{\mathcal{C}_{\mathcal{O}_{\mathbb{K}}}^{\text {ss }}}\left(M_{L}, M_{L^{\prime}}\right)=\operatorname{det}\left(L \mid L^{\prime}\right)$, if $L$ and $L^{\prime}$ are commensurable, and 0 otherwise. In Theorem 3.11, we constructed a gerbal representation $F$ of $G L_{\infty, \infty}$ on $\mathcal{C}_{\mathcal{O}_{\mathbb{K}}}^{\text {ss }}$. The obstruction to upgrade this gerbal representation to a genuine representation is a cohomology class in $H^{3}\left(G L_{\infty, \infty}, \mathcal{Z}\left(\mathcal{C}_{\mathcal{O}_{\mathbb{K}}}^{\text {ss }}\right)^{\times}\right)$(see Theorem 2.10). Since $\mathcal{C}_{\mathcal{O}_{\mathbb{K}}}^{\text {ss }}$ is $\mathbb{C}$-linear, there is a natural embedding $\mathbb{C}^{\times} \subset \mathcal{Z}\left(\mathcal{C}_{\mathcal{O}_{\mathbb{K}}}^{\text {ss }}\right)^{\times}$which admits a splitting. The second main theorem of this paper is

Theorem 5.9. The cohomology class corresponding to the gerbal representation of $G L_{\infty, \infty}$ on $\mathcal{C}_{\mathcal{O}_{\mathbb{K}}}^{\mathrm{ss}}$, as constructed in Theorem 3.11, is

$$
\left[E_{3}\right] \in H^{3}\left(G L_{\infty, \infty}, \mathbb{C}^{\times}\right) \subset H^{3}\left(G L_{\infty, \infty}, \mathcal{Z}\left(\mathcal{C}_{\mathcal{O}_{\mathbb{K}}}^{\text {ss }}\right)^{\times}\right),
$$

whose existence is proved in Theorem 5.7.

The strategy of proving this theorem is to realize this gerbal representation in another way, using gerbal pairs, so that we could apply results from 5.1 .3 to calculate the cohomology class.

Let us recall that we have the $\mathbb{C}^{\times}$-central extension of $\widehat{G L}_{\mathfrak{f}, \infty}$ (4.5),

$$
1 \rightarrow \mathbb{C}^{\times} \rightarrow \widehat{G L}_{\mathfrak{f}, \infty} \rightarrow G L_{\mathfrak{f}, \infty} \rightarrow 1
$$

On the other hand, we have the group extension (4.9),

$$
1 \rightarrow G L_{\mathfrak{f}, \infty} \rightarrow \widetilde{G L}_{\infty}\left(\mathfrak{g l}_{\infty}\right) \rightarrow G L_{\infty, \infty} \rightarrow 1
$$


Furthermore, there is an action of $\widetilde{G L}_{\infty}\left(\mathfrak{g l}_{\infty}\right)$ on $\widehat{G L}_{\mathfrak{f}, \infty}$ which leaves the central subgroup $\mathbb{C}^{\times} \subset \widehat{G L}_{\mathfrak{f}, \infty}$ invariant. Therefore, $\left(\widehat{G L}_{\infty}\left(\mathfrak{g l}_{\infty}\right), \widehat{G L}_{\mathfrak{f}, \infty}\right)$ is a gerbal pair, in the sense of Definition 5.1 (see Theorem 5.5 ).

Since $G L_{\infty}^{+}\left(\mathfrak{g l}_{\infty}\right)$ acts on $\mathcal{O}_{\mathbb{K}}$ (see formula (4.8)), it acts on the category of $\mathrm{Cl}_{\mathcal{O}_{\mathbb{K}}}$, modules. It is clear that $\mathcal{C}_{\mathcal{O}_{\mathbb{K}}}^{\mathrm{ss}}$ is invariant under this action. Indeed, $g \in G L_{\infty}^{+}\left(\mathfrak{g l}_{\infty}\right)$ will send $M_{L}$ to $M_{g L}$. Therefore, $\widetilde{G L}_{\infty}\left(\mathfrak{g l}_{\infty}\right)$ acts on $\mathcal{C}_{\mathcal{O}_{\mathbb{K}}}^{\text {ss }}$ via the surjection $\widetilde{G L}_{\infty}\left(\mathfrak{g l}_{\infty}\right) \rightarrow$ $G L_{\infty}^{+}\left(\mathfrak{g l}_{\infty}\right)$. We will denote this action by $G$ in what follows. If for any $a \in G L_{\mathfrak{f}, \infty} \subset$ $\widetilde{G L}_{\infty}\left(\mathfrak{g l}_{\infty}\right)$ we had an isomorphism $G_{a} \cong \mathbf{1}_{\mathcal{C}_{\mathcal{O}_{\mathbb{K}}}^{\text {ss }}}$, then we would have the sought-after second construction of gerbal representation of $G L_{\infty, \infty}$ on $\mathcal{C}_{\mathcal{O}_{\mathbb{K}}}^{\text {ss }}$, which would allow us to calculate its third cohomology class. However, this is not true, since $G_{a}$ is not always isomorphic to $\mathbf{1}_{\mathcal{C}_{\mathcal{O}_{\mathbb{K}}}^{\text {ss }}}$. Namely, from Example 4.12 in 44.2 .4 , we know that $a L$ and $L$ are not necessarily commensurable with each other, and therefore $G_{a}\left(M_{L}\right)=M_{a L}$ are not necessarily isomorphic to $M_{L}$. To remedy this problem, we will define a new semisimple abelian category $\widehat{\mathcal{C}}_{\mathcal{O}_{\mathbb{K}}^{\mathrm{ss}}}$, whose irreducible objects are still labeled by the secondary lattices $L \subset \mathcal{O}_{\mathbb{K}}$, but whose morphism sets are enlarged a little bit.

Let $\mathbb{L}$ be a lattice in $\mathbb{K}$ and $L, L^{\prime}$ two secondary lattices of $\mathbb{L}$ (see Definitions 3.3 and 3.6). Let us recall that we can $\operatorname{define} \operatorname{det}\left(L \mid L^{\prime}\right)$ if $L, L^{\prime}$ are pseudo commensurable with each other (see Definition 4.9). We will denote by $\widehat{\mathcal{C}_{\mathbb{L}}^{\text {ss }}}$ the semisimple abelian category, whose irreducible objects, denoted by $\widehat{M}_{L}$, are labeled by the secondary lattices $L \subset \mathbb{L}$ and whose morphism sets are

$$
\operatorname{Hom}_{\widehat{\mathbb{C}_{\mathbb{S}}}}\left(\widehat{M}_{L}, \widehat{M}_{L^{\prime}}\right)= \begin{cases}\operatorname{det}\left(L \mid L^{\prime}\right) & L, L^{\prime} \text { are pseudo commensurable } \\ 0 & \text { otherwise }\end{cases}
$$

Clearly, there is a full embedding $\Upsilon_{\mathbb{L}}: \mathcal{C}_{\mathbb{L}}^{\text {ss }} \rightarrow \widehat{\mathcal{C}}_{\mathbb{L}}^{\text {ss }}$, which sends $M_{L}$ to $\widehat{M}_{L}$.

Let us observe that the same construction as in Theorem 3.11 yields a gerbal representation of $G L_{\infty, \infty}$ on $\widehat{\mathcal{C}_{\mathbb{L}}^{\text {ss }}}$, which we will denote by $\widehat{F}$. The embedding $\Upsilon_{\mathbb{L}}$ commutes with the gerbal representations of $G L_{\infty, \infty}$, i.e. $\widehat{F}_{g} \circ \Upsilon_{\mathbb{L}} \cong \Upsilon_{\mathbb{L}} \circ F_{g}$. Therefore, by Proposition 2.12, it is enough to calculate the cohomology class corresponding to the gerbal representation $\widehat{F}$ of $G L_{\infty, \infty}$ on $\widehat{\mathcal{C}}_{\mathcal{O}_{\mathbb{K}}^{\text {s }}}^{\text {. }}$.

Now we give another realization of this gerbal representation. Observe that there is a genuine representation of $G L_{\infty}^{+}\left(\mathfrak{g l}_{\infty}\right)$ on $\widehat{\mathcal{C}}_{\mathcal{O}_{\mathbb{K}}}^{\mathrm{ss}}$. Namely, for $g \in G L_{\infty}^{+}\left(\mathfrak{g l}_{\infty}\right)$, it will send $\widehat{M}_{L}$ to $\widehat{M}_{g L}$ and $\operatorname{det}\left(L \mid L^{\prime}\right)$ to $\operatorname{det}\left(g L \mid g L^{\prime}\right)$. Therefore, we obtain a genuine representation of $\widetilde{G L}_{\infty}\left(\mathfrak{g l}_{\infty}\right)$ on $\widehat{\mathcal{C}}_{\mathcal{O}_{\mathbb{K}}}^{\text {ss }}$ via the surjection $\widetilde{G L}_{\infty}\left(\mathfrak{g l}_{\infty}\right) \rightarrow G L_{\infty}^{+}\left(\mathfrak{g l}_{\infty}\right)$, which is denoted by $\widehat{G}$ in what follows. Furthermore, our definition of $\widehat{\mathcal{C}}_{\mathcal{O}_{\mathbb{K}}}^{\mathrm{ss}}$ makes, for any $a \in G L_{\mathfrak{f}, \infty}$, $G_{a} \cong \mathbf{1}_{\widehat{\mathcal{C}}_{\mathcal{O}_{\mathbb{K}}}}$. Indeed, recall the $\mathbb{C}^{\times}$-central extension $\widehat{G L}_{\mathfrak{f}, \infty} \stackrel{\pi}{\rightarrow} G L_{\mathfrak{f}, \infty}$. Thus, $\pi^{-1}(a)$ is a $\mathbb{C}^{\times}$-torsor for any $a \in G L_{\mathfrak{f}, \infty}$.

Lemma 5.10. There is a $\mathbb{C}^{\times}$-equivariant embedding

$$
\pi^{-1}(a) \rightarrow \operatorname{Hom}_{\mathbb{G L}\left(\widehat{\mathcal{C}}_{\mathcal{O}_{\mathbb{K}}}^{\mathrm{ss}}\right)}\left(G_{a}, \mathbf{1}_{\widehat{\mathcal{C}_{\mathcal{O}_{\mathbb{K}}}^{\mathrm{ss}}}}\right)
$$

such that the assumptions of Proposition 5.4 hold.

Proof. Let us fix a secondary lattice $L \subset \mathcal{O}_{\mathbb{K}}$. For simplicity, we will use $L_{0}=\mathbb{C}[[t]][[s]]$. By Proposition 4.13, $\pi^{-1}(a)$ may be identified with $\operatorname{det}\left(a L_{0} \mid L_{0}\right)^{\times}$. We have constructed in Theorem 5.5 an action $\tilde{\rho}$ of $G L_{\infty}^{+}\left(\mathfrak{g l}_{\infty}\right)$ on $\widehat{G L}_{\mathfrak{f}, \infty}$. This action gives for each $a$ an 
isomorphism

$$
\gamma_{a, g}: \operatorname{det}\left(a L_{0} \mid L_{0}\right) \stackrel{\tilde{\rho}_{g^{-1}}}{\rightarrow} \operatorname{det}\left(\rho_{g^{-1}}(a) L_{0} \mid L_{0}\right) \stackrel{g}{\rightarrow} \operatorname{det}\left(a g L_{0} \mid g L_{0}\right)
$$

If $g$ fixes $L_{0}$, then $\gamma_{a, g}$ is just multiplication of a non-zero number. We claim that in this case $\gamma_{a, g}=\mathrm{id}$. To prove this, let $R$ be the subalgebra of $\mathfrak{g l}_{\infty}$ consisting of $A: K \rightarrow K$ such that $A\left(\mathcal{O}_{K}\right) \subset \mathcal{O}_{K}$ (recall that $\left.K=\mathbb{C}((t)), \mathcal{O}_{K}=\mathbb{C}[[t]]\right)$. Then $G L_{\infty}^{+}(R)$ is the subgroup of $G L_{\infty}^{+}\left(\mathfrak{g l}_{\infty}\right)$ that fixes $L_{0}$. Now $\gamma_{a, g} \gamma_{a, g^{\prime}}=\gamma_{a, g g^{\prime}}$ for $g, g^{\prime} \in G L_{\infty}^{+}(R)$. But from Proposition 4.2, $\gamma_{a, g}=$ id for $a \in G L_{\infty}^{+}(R)$.

Let $L$ be any secondary lattice of $\mathcal{O}_{\mathbb{K}}$. By Lemma 4.10, we can choose $g \in G L_{\infty}^{+}\left(\mathfrak{g l}_{\infty}\right)$ such that $g L_{0}=L$, and therefore obtain an isomorphism $\gamma_{a, g}: \operatorname{det}\left(a L_{0} \mid L_{0}\right) \cong \operatorname{det}(a L \mid L)$, which is independent of the choice of $g$ by previous discussion. Therefore, we obtain for any $a \in G L_{\mathfrak{f}, \infty}, L \in \widehat{\mathcal{C}}_{\mathcal{O}_{\mathbb{K}}^{\mathrm{ss}}}^{\mathrm{s}}$, an isomorphism

$$
\pi^{-1}(a) \rightarrow \operatorname{det}\left(a L_{0} \mid L_{0}\right) \rightarrow \operatorname{det}(a L \mid L)=\operatorname{Hom}_{\widehat{\mathcal{C}}_{\mathcal{O}_{\mathbb{K}}}}\left(\widehat{M}_{a L}, \widehat{M}_{L}\right) .
$$

It is easy to check that these isomorphisms give the desired map (5.5) such that the assumptions of Proposition 5.4 hold.

Applying Proposition 5.4, we obtain a gerbal representation $\widehat{G}$ of $G L_{\infty, \infty}$ on $\widehat{\mathcal{C}}_{\mathcal{O}_{\mathbb{K}}}$. The cohomology class corresponding to this gerbal representation is $\left[E_{3}\right] \in H^{3}\left(G L_{\infty, \infty}, \mathbb{C}^{\times}\right)$ as claimed in Theorem 5.7 .

To complete the proof of Theorem 5.9, we need the following:

Lemma 5.11. The two gerbal representations, $\widehat{F}$ and $\widehat{G}$, of $G L_{\infty, \infty}$ on $\widehat{\mathcal{C}}_{\mathcal{O}_{\mathbb{K}}^{\mathrm{ss}}}$ are equivalent, that is, for any $g \in G L_{\infty, \infty}, \widehat{F}_{g} \cong \widehat{G}_{g}$.

Proof. Let $g \in G L_{\infty, \infty}$, and $L \subset \mathcal{O}_{\mathbb{K}}$ be a secondary lattice. It is clear that we only need to show that $\widehat{F}_{g}\left(\widehat{M}_{L}\right) \cong \widehat{G}_{g}\left(\widehat{M}_{L}\right)$.

First, $\widehat{F}_{g}\left(\widehat{M}_{L}\right) \cong \widehat{M}_{L^{\prime}}$, where $L^{\prime}$ is a secondary lattice of $\mathcal{O}_{\mathbb{K}}$ fitting into the following exact sequence

$$
0 \rightarrow g L \cap \mathcal{O}_{\mathbb{K}} \rightarrow L^{\prime} \rightarrow V_{\mathcal{O}_{\mathbb{K}} \cap g \mathcal{O}_{\mathbb{K}}, \mathcal{O}_{\mathbb{K}}} \rightarrow 0 .
$$

(We recall that $V_{\mathcal{O}_{\mathbb{K}} \cap g \mathcal{O}_{\mathbb{K}}, \mathcal{O}_{\mathbb{K}}}$ was introduced in 33.3.1.) On the other hand, $\widehat{G}_{g}\left(\widehat{M}_{L}\right) \cong$ $\widehat{M}_{a L}$, where $a \in G L_{\infty}^{+}\left(\mathfrak{g l}_{\infty}\right)$ such that $(a, g) \in \widetilde{G L}_{\infty}\left(\mathfrak{g l}_{\infty}\right)$. Let us recall that the definition of $\widetilde{G L}{ }_{\infty}\left(\mathfrak{g l}_{\infty}\right)$ (see 4 .1.2) implies that $(a, g) \in G L_{\infty}^{+}\left(\mathfrak{g l}_{\infty}\right) \times G L_{\infty, \infty}$ is in $\widetilde{G L}_{\infty}\left(\mathfrak{g l}_{\infty}\right)$ if and only if $\exists n$ such that $g\left(s^{n} \mathcal{O}_{\mathbb{K}}\right) \subset \mathcal{O}_{\mathbb{K}}$ and $\left.a\right|_{s^{n} \mathcal{O}_{\mathbb{K}}}=\left.g\right|_{s^{n} \mathcal{O}_{\mathbb{K}}}$. Therefore, $a L \cap a s^{n} \mathcal{O}_{\mathbb{K}}=g L \cap g s^{n} \mathcal{O}_{\mathbb{K}}=L^{\prime} \cap g s^{n} \mathcal{O}_{\mathbb{K}}=L^{\prime} \cap a s^{n} \mathcal{O}_{\mathbb{K}}$. That is, $a L$ and $L^{\prime}$ are pseudo commensurable with each other. Therefore, $\widehat{F}_{g}\left(\widehat{M}_{L}\right) \cong \widehat{G}_{g}\left(\widehat{M}_{L}\right)$.

Theorem 5.9 is now proved. As a corollary, we obtain that the third cohomology class defined by the determinantal $B \mathbb{C}^{\times}$-extension $\mathbb{G} \mathbb{L}_{\infty, \infty}$ of $G L_{\infty, \infty}$ is also equal to $\left[E_{3}\right]$.

5.3.2. The gerbal representation of $G L_{\infty, \infty}$ on the category of representations of $\widehat{G L}_{\mathfrak{f}, \infty}$. We can also realize the cohomology class $\left[E_{3}\right] \in H^{3}\left(G L_{\infty, \infty}, \mathbb{C}^{\times}\right)$as the one corresponding to the gerbal representation of $G L_{\infty, \infty}$ on a category of representations of $\widehat{G L}_{\mathfrak{f}, \infty}$.

Denote by $\operatorname{Rep}_{1}\left(\widehat{G L}_{\mathfrak{f}, \infty}\right)$ the category of level one complex representations of $\widehat{G L}_{\mathfrak{f}, \infty}$, i.e, those representations on which the central $\mathbb{C}^{\times}$acts via the standard character. As shown in $\$ 5.1 .3$, we have a gerbal representation of $G L_{\infty, \infty}$ on the category of $\operatorname{Rep}_{1}\left(\widehat{G L}_{\mathfrak{f}, \infty}\right)$. The natural embedding $\mathbb{C}^{\times} \subset \mathcal{Z}\left(\operatorname{Rep}_{1}\left(\widehat{G L}_{\mathfrak{f}, \infty}\right)\right)^{\times}$admits a splitting, 
and therefore the induced map $H^{3}\left(G L_{\infty, \infty}, \mathbb{C}^{\times}\right) \rightarrow H^{3}\left(G L_{\infty, \infty}, \mathcal{Z}\left(\operatorname{Rep}_{1}\left(\widehat{G L}_{\mathfrak{f}, \infty}\right)\right)^{\times}\right)$is injective. Combining this with Theorem [5.7, we obtain that the cohomology class corresponding to this gerbal representation is just $\left[E_{3}\right] \in H^{3}\left(G L_{\infty, \infty}, \mathbb{C}^{\times}\right)$.

5.3.3. Level one representations of $\widehat{G L}_{\mathfrak{f}, \infty}$. In this subsection we sketch a construction of some level one representations of $\widehat{G L}_{\mathfrak{f}, \infty}$, using modules over a Clifford algebra. They form a subcategory of $\operatorname{Rep}_{1}\left(\widehat{G L}_{\mathfrak{f}, \infty}\right)$, which is invariant under the gerbal action of $G L_{\infty, \infty}$. Therefore, the gerbal representation of $G L_{\infty, \infty}$ on this subcategory also realizes the class $\left[E_{3}\right]$. This subsection is independent from the rest of the paper, and its main goal is to describe a conjectural relation between the categories $\operatorname{Rep}_{1}\left(\widehat{G L}_{\mathfrak{f}, \infty}\right)$ and $\widehat{\mathcal{C}}_{\mathcal{O}_{\mathbb{K}}}^{\text {ss }}$.

Recall that in $\oiint 1$ we constructed level one representations of $\widehat{G L}_{\infty}$ by using discrete Clifford modules over $\mathrm{Cl}_{K}$. We would like to make similar constructions here to obtain some interesting level one representations of $\widehat{G L}_{\mathfrak{f}, \infty}$. Note however that these representations are no longer discrete.

Recall the notation $\mathbb{K}=\mathbb{C}((t))((s))$ and $\mathbb{K}^{*}=\mathbb{C}((t))((s)) d t d s$. The natural pairing on $\mathbb{K} \oplus \mathbb{K}^{*}$ is given by

$$
(f, \omega)=\operatorname{Res}_{t=0} \operatorname{Res}_{s=0} f \omega \quad f \in \mathbb{K}, \omega \in \mathbb{K}^{*} .
$$

Recall that $\mathcal{O}_{\mathbb{K}}=\mathbb{C}((t))[[s]]$ and $\mathrm{Cl}_{\mathcal{O}_{\mathbb{K}}}=\mathrm{Cl}\left(\mathcal{O}_{\mathbb{K}} \oplus \mathbb{K}^{*} / \mathcal{O}_{\mathbb{K}}^{\perp}\right)$ is the corresponding Clifford algebra. We define the action of $G L_{\mathfrak{f}, \infty}$ on $\mathcal{O}_{\mathbb{K}}$ via formula (4.8) and on $\mathbb{K}^{*} / \mathcal{O}_{\mathbb{K}}^{\perp}$ so that the bilinear form on $\mathcal{O}_{\mathbb{K}} \oplus \mathbb{K}^{*} / \mathcal{O}_{\mathbb{K}}^{\perp}$ is $G L_{\mathfrak{f}, \infty}$-invariant.

Let $L$ be a secondary lattice of $\mathcal{O}_{\mathbb{K}}$ (see Definition 3.6). We have constructed in 3.2.2 the vacuum module $M_{L}$ over $\mathrm{Cl}_{\mathcal{O}_{\mathbb{K}}}$. However, since for $a \in G L_{\mathfrak{f}, \infty}, M_{a L}$ and $M_{L}$ are not necessarily isomorphic as $\mathrm{Cl}_{\mathcal{O}_{\mathbb{K}}}$-modules, the construction we used in $\$ 1.3 .3$ for $G L_{\infty}$ cannot not be applied here. Instead, it turns out that a certain completion of $M_{L}$ carries the action of $\widehat{G L}_{\mathfrak{f}, \infty}$.

Let $L_{0}=\mathbb{C}[[t]][[s]]$ and $L_{0}^{\prime}=t^{-1} \mathbb{C}\left[t^{-1}\right][[s]]$, so that $\mathcal{O}_{\mathbb{K}}=L_{0} \oplus L_{0}^{\prime}$. Then

$$
M_{L_{0}}=\operatorname{Ind}_{\bigwedge\left(L_{0} \oplus L_{0}^{\perp}\right)}^{\mathrm{Cl}_{\mathcal{O}_{\mathbb{K}}}} \mathbb{C}|0\rangle \cong \bigwedge\left(L_{0}^{\prime} \oplus L_{0}^{\prime \perp}\right) \text {. }
$$

There is a natural topology on $\bigwedge\left(L_{0}^{\prime} \oplus L_{0}^{\prime \perp}\right)$. Namely, a basis of open neighborhoods of 0 can be chosen as $\bigwedge\left(L_{0}^{\prime} \oplus L_{0}^{\prime \perp}\right)\left(s^{n} \mathcal{O}_{\mathbb{K}} \cap L_{0}^{\prime}\right)$. The completion of $M_{L_{0}}$ with respect to this topology is denoted by $\widehat{M}_{L_{0}, L_{0}^{\prime}}$. This is a completed Clifford module over a certain completed Clifford algebra. Namely, we denote by $\overline{\mathrm{Cl}}_{\mathcal{O}_{\mathbb{K}}, L_{0}^{\prime}}$ the completion of $\mathrm{Cl}_{\mathcal{O}_{\mathbb{K}}}$ with respect to the topology in which a basis of open neighborhoods of 0 is given by the subspaces $U+V$, where $U$ is the left ideal generated by open subspaces of $L_{0} \oplus L_{0}^{\perp}$ and $V$ is the right ideal generated by $s^{n} \mathcal{O}_{\mathbb{K}} \cap L_{0}^{\prime}$. It is clear that the multiplication of $\mathrm{Cl}_{\mathcal{O}_{\mathbb{K}}}$ extends to a multiplication of $\overline{\mathrm{Cl}}_{\mathcal{O}_{\mathbb{K}}, L_{0}^{\prime}}$. Let $\bigwedge\left(\widehat{L_{0} \oplus L_{0}^{\perp}}\right)$ be the subalgebra of $\overline{\mathrm{Cl}}_{\mathcal{O}_{\mathbb{K}}, L_{0}^{\prime}}$ topologically generated by $L_{0} \oplus L_{0}^{\perp}$. Then

$$
\widehat{M}_{L_{0}, L_{0}^{\prime}}=\operatorname{Ind} \underset{\wedge\left(\overline{\mathrm{Cl}}_{\mathcal{O}_{\mathbb{K}}, L_{0}^{\prime}}\right.}{ } \mathbb{C}|0\rangle .
$$

The reason that $\widehat{M}_{L_{0}, L_{0}^{\prime}}$ is a representation of $\widehat{G L}_{\mathfrak{f}, \infty}$ is based on the following

Lemma 5.12. Let $L_{1}$ be a secondary lattice of $\mathcal{O}_{\mathbb{K}}$ that is pseudo commensurable with $L_{0}$ (i.e., $\exists n$ such that $\left.s^{n} \mathcal{O}_{\mathbb{K}} \cap L_{1}=s^{n} \mathcal{O}_{\mathbb{K}} \cap L_{0}\right)$. Let $\bigwedge\left(\widehat{L_{1} \oplus L_{1}^{\perp}}\right)$ be the subalgebra of 
$\overline{\mathrm{Cl}}_{\mathcal{O}_{\mathbb{K}}, L_{0}^{\prime}}$ that is topologically generated by $L_{1} \oplus L_{1}^{\perp}$, and let

$$
\widehat{M}_{L_{1}, L_{0}^{\prime}}=\operatorname{Ind} \underset{\bigwedge\left(\bar{L}_{L_{1} \oplus L_{1}^{\perp}}\right)}{\overline{\mathrm{O}}_{\mathbb{K}, L_{0}^{\prime}}} \mathbb{C}|0\rangle .
$$

Then $\widehat{M}_{L_{1}, L_{0}^{\prime}} \cong \widehat{M}_{L_{0}, L_{0}^{\prime}}$ and there is a canonical isomorphism

$$
\operatorname{Hom}_{\overline{\mathrm{Cl}}_{\mathcal{O}_{\mathbb{K}}, L_{0}^{\prime}}}\left(\widehat{M}_{L_{1}, L_{0}^{\prime}}, \widehat{M}_{L_{0}, L_{0}^{\prime}}\right)=\operatorname{det}\left(L_{1} \mid L_{0}\right)
$$

such that if $L_{2}$ is another secondary lattice that is pseudo commensurable with $L_{0}, L_{1}$, then similar diagram as in Lemma 1.11 holds.

Let us sketch the proof of this lemma. First, we identify $\operatorname{Hom}_{\overline{\mathrm{Cl}}_{\mathcal{O}_{\mathbb{K}}, L_{0}^{\prime}}}\left(\widehat{M}_{L_{1}, L_{0}^{\prime}}, \widehat{M}_{L_{0}, L_{0}^{\prime}}\right)$ with $\left(\widehat{M}_{L_{0}, L_{0}^{\prime}}\right)^{\wedge\left(\widehat{L_{1} \oplus L} \frac{\perp}{1}\right)}$, the $\bigwedge\left(\widehat{L_{1} \oplus L_{1}^{\perp}}\right)$-invariant subspace of $\widehat{M}_{L_{0}, L_{0}^{\prime}}$. It is easy to see that $\left(\widehat{M}_{L_{0}, L_{0}^{\prime}}\right)^{\wedge\left({\widehat{L_{0} \oplus L}}_{0}^{\perp}\right)}=\mathbb{C}|0\rangle$. Next, one can construct an injective map

$$
\operatorname{det}\left(L_{1} \mid L_{0}\right) \rightarrow\left(\widehat{M}_{L_{0}, L_{0}^{\prime}}\right) \wedge\left(\widehat{L_{1} \oplus L} \frac{1}{1}\right)
$$

Indeed, one can apply Lemma 1.11 to construct for any $m \geq n$, an embedding

$$
\operatorname{det}\left(\frac{L_{1}}{L_{1} \cap s^{m} \mathcal{O}_{\mathbb{K}}} \mid \frac{L_{0}}{L_{0} \cap s^{m} \mathcal{O}_{\mathbb{K}}}\right) \rightarrow \widehat{M}_{L_{0}, L_{0}^{\prime}} /\left(s^{m} \mathcal{O}_{\mathbb{K}} \cap L_{0}^{\prime}\right) \widehat{M}_{L_{0}, L_{0}^{\prime}}
$$

in a compatible way. The projective limit of this system of maps is the desired one. Therefore, one obtains an embedding

$$
\varphi: \operatorname{det}\left(L_{1} \mid L_{0}\right) \rightarrow \operatorname{Hom}_{\overline{\mathrm{Cl}}_{\mathcal{O}_{\mathbb{K}}, L_{0}^{\prime}}}\left(\widehat{M}_{L_{1}, L_{0}^{\prime}}, \widehat{M}_{L_{0}, L_{0}^{\prime}}\right)
$$

Finally, one can check for $c \in \operatorname{det}\left(L_{1} \mid L_{0}\right)^{\times}, \varphi(c)$ is an isomorphism. Therefore, $\left(\widehat{M}_{L_{0}, L_{0}^{\prime}}\right)^{\wedge\left({\widehat{L_{0} \oplus L}}_{0}^{\perp}\right)}=\mathbb{C}|0\rangle$ forces that $\varphi$ is an isomorphism. This completes the sketch of proof of the lemma.

Now observe that the action of $G L_{\mathfrak{f}, \infty}$ on $\mathrm{Cl}_{\mathcal{O}_{\mathbb{K}}}$ is continuous with respect to the topology we have introduced. Therefore, $G L_{\mathfrak{f}, \infty}$ acts on $\overline{\mathrm{Cl}}_{\mathcal{O}_{\mathbb{K}}, L_{0}^{\prime}}$. The same construction as in $\$ 1$ applies here and gives $\widehat{M}_{L_{0}, L_{0}^{\prime}}$ the structure of a representation of $\widehat{G L}_{\mathfrak{f}, \infty}$. From this representation we construct a subcategory of level one representations of $\widehat{G L}_{\mathfrak{f}, \infty}$. Recall that $G L_{\infty}^{+}\left(\mathfrak{g l}_{\infty}\right)$ acts on $\operatorname{Rep}_{1}\left(\widehat{G L}_{\mathfrak{f}, \infty}\right)$. Denote this action by $H$. Therefore, for each $g \in G L_{\infty}^{+}\left(\mathfrak{g l}_{\infty}\right)$, there is a level one representations of $\widehat{G L}_{\mathfrak{f}, \infty}, H_{g}\left(\widehat{M}_{L_{0}, L_{0}^{\prime}}\right)$. The semisimple abelian category generated by these objects, which is denoted by $\mathfrak{R e p}$, is the "smallest" abelian subcategory of $\operatorname{Rep}_{1}\left(\widehat{G L}_{\mathfrak{f}, \infty}\right)$ that contains $\widehat{M}_{L_{0}, L_{0}^{\prime}}$ and is invariant under the gerbal representation of $G L_{\infty, \infty}$ on $\operatorname{Rep}_{1}\left(\widehat{G L}_{\mathfrak{f}, \infty}\right)$. It is clear that the gerbal representation of $G L_{\infty, \infty}$ on $\mathfrak{R e p}$ realizes $\left[E_{3}\right]$.

Finally, we explain a conjectural connection between $\mathfrak{R e p}$ and $\widehat{\mathcal{C}}_{\mathcal{O}_{\mathbb{K}}^{s s}}$. Observe that in order to define the completed Clifford algebra $\overline{\mathrm{Cl}}_{\mathcal{O}_{\mathbb{K}}, L_{0}^{\prime}}$, we had to choose a splitting $\mathcal{O}_{\mathbb{K}}=L_{0} \oplus L_{0}^{\prime}$, which we used to define a topology on $\mathrm{Cl}_{\mathcal{O}_{\mathbb{K}}}$. If we choose a different splitting $\mathcal{O}_{\mathbb{K}}=L_{0} \oplus L_{0}^{\prime \prime}$, we will obtain a different completed Clifford algebra $\overline{\mathrm{Cl}}_{\mathcal{O}_{\mathbb{K}}, L_{0}^{\prime \prime}}$ and the module $\widehat{M}_{L_{0}, L_{0}^{\prime \prime}}$ over it. However, for different choices of splitting $\mathcal{O}_{\mathbb{K}}$, the corresponding completed Clifford algebras are canonically isomorphic. Namely, they are all isomorphic to the completed Clifford algebra modeled on the orthogonal topological 
vector space $\left(L_{0} \oplus L_{0}^{\perp}\right) \oplus\left(\mathcal{O}_{\mathbb{K}} / L_{0} \oplus\left(\mathbb{K}^{*} / \mathcal{O}_{\mathbb{K}}^{\perp}\right) / L_{0}^{\perp}\right)$. Furthermore, both $M_{L_{0}, L_{0}^{\prime}}$ and $M_{L_{0}, L_{0}^{\prime \prime}}$ are identified with the vacuum module induced from the trivial module over $\bigwedge\left(L_{0} \oplus L_{0}^{\perp}\right)$. The real reason that the splitting may potentially come into the play is that the action of $G L_{\mathfrak{f}, \infty}$ on this completed Clifford algebra depends on the choice of the splitting. Therefore, a priori, $\widehat{M}_{L_{0}, L_{0}^{\prime \prime}}$ and $\widehat{M}_{L_{0}, L_{0}^{\prime}}$ may not be isomorphic as representations of $\widehat{G L}_{\mathfrak{f}, \infty}$. However, we expect that these two representations are indeed canonically isomorphic. If this is the case, then we can drop the subscript $L_{0}^{\prime}$ and simply denote them by $\widehat{M}_{L_{0}}$. Now the conjectural connection between $\widehat{\mathcal{C}}_{\mathcal{O}_{\mathbb{K}}}^{\text {ss }}$ and $\mathfrak{R e p}$ is the following: there is a $G L_{\infty}^{+}\left(\mathfrak{g l}_{\infty}\right)$-equivariant equivalence of categories $\widehat{\mathcal{C}}_{\mathcal{O}_{\mathbb{K}}}^{\text {ss }} \rightarrow \mathfrak{R e p}$ which sends the object $\widehat{M}_{L_{0}}$ in $\widehat{\mathcal{C}}_{\mathcal{O}_{\mathbb{K}}^{\text {ss }}}$ to the same named object in $\mathfrak{R e p}$. Hence we obtain a homomorphism of the corresponding gerbal representations of $G L_{\infty, \infty}$.

\section{REFERENCES}

[ADK] E. Arbarello, C. De Concini and V. Kac, The infinite wedge representation and the reciprocity law for algebraic curves, in Theta functions - Bowdoin 1987, Part 1 (Brunswick, ME, 1987), pp. 171-190, Proc. Sympos. Pure Math. 49, Part 1, Amer. Math. Soc., Providence, RI, 1989.

[AK] S. Arkhipov and K. Kremnizer, 2-gerbes and 2-Tate spaces, Preprint arXiv:0708.4401,

[BL] J.C. Baez and A.D. Lauda, Higher-dimensional algebra V: 2-groups, Theory Appl. Categ. 12 (2004) 423-491 (electronic).

[BBE] A. Beilinson, S. Bloch and H. Esnault, $\epsilon$-factors for Gauss-Manin determinants, Mosc. Math. J. 2 (2002) 477-532.

[BD] A. Beilinson and V. Drinfeld, Chiral algebras, American Mathematical Society Colloquium Publications 51, AMS, 2004.

[BBS] S. Berman, Y. Billig and J. Szmigielski, Vertex operator algebras and the representation theory of toroidal algebras, in Recent developments in infinite-dimensional Lie algebras and conformal field theory (Charlottesville, VA, 2000), pp. 1-26, Contemp. Math. 297, Amer. Math. Soc., Providence, RI, 2002.

[Be] R. Bezrukavnikov, On tensor categories attached to cells in affine Weyl groups, Representation theory of algebraic groups and quantum groups, 69-90, Adv. Stud. Pure Math., 40, Math. Soc. Japan, Tokyo, 2004.

[Bi] Yu. Billig, Representations of toroidal extended affine Lie algebras, J. Algebra 308 (2007) 252-269.

[Br] L. Breen, Théorie de Schreier supérieure, Ann. Sci. École Norm. Sup. (4) 25 (1992), no. 5, $465-514$.

[DJKM] E. Date, M. Jimbo, M. Kashiwara and T. Miwa, Transformation groups for soliton equations, in Non-linear Integrable Systems - Classical Theory and Quantum Theory, M. Jimbo, T. Miwa (eds.), pp. 39-120, World Scientific, Singapore, 1983.

[Dr] V. Drinfeld, Infinite-dimensional vector bundles in algebraic geometry: an introduction, in The unity of mathematics, pp. 263-304, Progr. Math. 244, Birkhäuser Boston, Boston, MA, 2006.

[FW] F.T. Farrell and J.B. Wagoner, Infinite matrices in algebraic K-theory and topology, Comment. Math. Helv. 47 (1972) 474-501.

[Fe] B.L. Feigin, On the cohomology of the Lie algebra of vector fields and of the current algebra, Selecta Math. Sov. 7 (1988) 49-62.

[FT1] B.L. Feigin and B.L. Tsygan, Cohomology of Lie algebras of generalized Jacobi matrices, Funktsional. Anal. i Prilozhen. 17, no. 2 (1983) 86-87.

[FT2] B.L. Feigin and B.L. Tsygan, Cyclic homology of algebras with quadratic relations, universal enveloping algebras and group algebras, in K-theory, arithmetic and geometry (Moscow, 19841986), pp. 210-239, Lecture Notes in Math. 1289, Springer, Berlin, 1987.

[F] D. Freed, Remarks on Chern-Simons theory, Preprint arXiv:0808.2507.

[Fr] E. Frenkel, Langlands Correspondence for Loop Groups, Cambridge Studies in Advanced Mathematics 103, Cambridge University Press, 2007. 
[FB] E. Frenkel and D. Ben-Zvi, Vertex Algebras and Algebraic Curves, Mathematical Surveys and Monographs 88, Second Edition, AMS, 2004.

[FG] E. Frenkel and D. Gaitsgory, Local geometric Langlands correspondence and affine Kac-Moody algebras, in Algebraic geometry and number theory, pp. 69-260, Progr. Math. 253, Birkhäuser Boston, Boston, MA, 2006.

[FZ] E. Frenkel and X. Zhu, Gerbal representations of Lie algebras, to appear.

[Fre] I. Frenkel, B., Spinor representations of affine Lie algebras, Proc. Nat. Acad. Sci. U.S.A. 77 (1980), no. 11, part 1, 6303-6306.

[HS] G. Hochschild and J.-P. Serre, Cohomology of group extensions, Trans. Amer. Math. Soc. 74 (1953) 110-134.

[K] V.G. Kac, Infinite-dimensional Lie Algebras, 3rd Edition, Cambridge University Press, 1990.

[KP] V. Kac and D. Peterson, Spin and wedge representations of infinite-dimensional Lie algebras and groups, Proc. Nat. Acad. Sci. U.S.A. 78 (1981), no. 6, part 1, 3308-3312.

[Kap] M. Kapranov, Semiinfinte symmetric powers, Preprint arXiv:math.QA/0107089.

[La] T. Larsson, Extended diffeomorphism algebras and trajectories in jet space, Commun. Math. Phys. 214 (2000) 469-491.

[Li] M. Lieblich, Moduli Of Twisted Sheaves, Duke Math. J. 138 (2007) 23-118.

[MRY] R.V. Moody, S.E. Rao and T. Yokonuma, Toroidal Lie algebras and vertex representations, Geom. Dedicata 35 (1990) 283-307.

[Osi1] D. Osipov, Central extensions and reciprocity laws on algebraic surfaces, Preprint arXiv:math.0501155.

[Osi2] D. Osipov, Adeles on n-dimensional schemes and categories $C_{n}$, Preprint arXiv:math.AG/0509189

[PS] A.N. Pressley and G.B. Segal, Loop groups and their representations, Oxford Univerisity Press, 1985.

[Q] D. Quillen, Higher algebraic K-thoery, I, Lecture Notes in Mathematics 341, pp. 85-147, Springer 1973

[SW] G. Segal and G. Wilson, Loop groups and equations of KdV type, Publ. Math. IHES 63 (1985) $5-65$.

[S] H.X. Sinh, Gr-catégories, Thése, Univ. Paris VII, 1975.

[W] J.B. Wagoner, Delooping classifying spaces in algebraic K-theory, Topology 11 (1972) 349370.

Department of Mathematics, University of California, Berkeley, CA 94720, USA 